\title{
1 On the psychological origins of tool use
}

3 Madhur Mangalam ${ }^{1}$, Dorothy M. Fragaszy ${ }^{2}$, Jeffrey B. Wagman ${ }^{3}$, Brian M. Day ${ }^{4}$, Damian G. Kelty4 Stephen $^{5}$, Raoul M. Bongers ${ }^{6}$, Dietrich W. Stout ${ }^{7}$, and François Osiurak ${ }^{8,9}$

6 'Department of Physical Therapy, Movement and Rehabilitation Science, Northeastern University, 7 Boston, Massachusetts 02115, USA

8 'Department of Psychology, University of Georgia, Athens, GA 30602, USA

9 '3epartment of Psychology, Illinois State University, Normal, IL 61761, USA

10 '4Department of Psychology, Butler University, Indianapolis, IN 46208, USA

11 5Department of Psychology, Grinnell College, Grinnell, IA 50112, USA

12 'Department of Human Movement Sciences, University Medical Center Groningen, University of 13 Groningen, $9713 \mathrm{GZ}$ Groningen, Netherlands

$14{ }^{7}$ Department of Anthropology, Emory University, Atlanta, GA 30322, USA

$15{ }^{8}$ Laboratoire d'Etude des Mécanismes Cognitifs, Université de Lyon, Lyon 69361, France

$16{ }^{9}$ Institut Universitaire de France, Paris 75231, France 


\section{Abstract}

18 The ubiquity of tool use in human life has generated multiple lines of scientific and philosophical 19 investigation to understand the development and expression of humans' engagement with tools and its 20 relation to other dimensions of human experience. However, existing literature on tool use faces 21 several epistemological challenges in which the same set of questions generate many different 22 answers. At least four critical questions can be identified, which are intimately intertwined-(1) What 23 constitutes tool use? (2) What psychological processes are involved in tool use? (3) Which of these 24 psychological processes are specific to tool use? (4) Which psychological processes involved in tool 25 use are specific to Homo sapiens? To help advance a multidisciplinary scientific understanding of tool 26 use, six author groups representing different academic disciplines (e.g., anthropology, psychology, 27 neuroscience) and different theoretical perspectives respond to each of these questions, and then 28 point to the direction of future work on tool use. We find that while there are marked differences among 29 the responses of the respective author groups to each question, there is a-perhaps surprising30 degree of agreement about many essential concepts and questions. We believe that this 31 interdisciplinary and intertheoretical discussion will foster a more comprehensive understanding of tool 32 use than any one of these perspectives (or any one of these author groups) would (or could) on their 33 own.

34 Keywords: affordance; dexterity; perception; technical reasoning; tooling 


\section{Introduction}

The ubiquity of tools in human cultures since the origin of the genus Homo has provoked enduring philosophical inquiries into humans' engagement with tools (Gibson \& Ingold, 1994), in part because tool use is often considered to reflect a unique dimension of technical intelligence (Preston, 2012). Aristotle wrote more than 2,000 years ago, "The hand can become a claw, a fist, a horn or spear or sword or any other weapon or tool. It can be everything because it can grasp anything or hold anything" (Aristotle, The Animal Parts (IV, 10)). Today, anthropologists, ethologists, psychologists, and neuroscientists investigate the development and expression of humans' engagement with tools in relation to other dimensions of human experience, and the increased efficiency and complexity of tool use in human populations over generations (Boyd and Richerson, 1996; Osiurak and Reynaud, 2020a; Tomasello et al., 1993). The origin of this phenomenon, called cumulative technological culture, was judged in 2005 by the journal Science as one of the 125 big scientific questions of the millennium. It appears that deepening our understanding of the psychological bases of tool use is fundamental to understanding th origins of tool technological culture. One might conjecture at first glance he answers would be within easy reach. After all, they only require conceiving experiments in which individuals use tools, with researchers manipulating different variables to reveal the underlying psychological processes. However, behind this apparent simplicity, research on tool use faces several epistemological challenges in which the same set of questions generate many different answers. In this work, six research groups answer four critical questions about tool use, which are intimately intertwined, and suggest what should be the direction of future research on tool use. We provide a brief overview of the four questions before presenting each group's answers.

What constitutes tool use? This question may appear trivial yet it is not. The process of defining any psychological concept is neither neutral nor viewpoint-free-any definition is necessarily limited and oriented toward the theoretical solutions provided to account for the phenomenon studied (Osiurak and Heinke, 2018). We have often posed this question to our friends, and their responses have been quite varied. Some people exclusively reserve the term "tool use" for the use of handheld objects to solve a mechanical problem (e.g., a hammer to drive in a nail). Others referred to using any handheld implement (e.g., a calculator and a smartphone). Some considered any use of an object as tool use, irrespective of how it is used (e.g., a paperweight, a house). Some even argued that wearing clothing qualifies as tool use because it achieves the goal of avoiding sunburn or regulating body temperature. The responses have been all over the place. (The readers may try asking the same question to their friends over a beer some evening!). The same difficulties also arise in the scientific community, in which there is no strict consensus on what tool use is (Fragaszy \& Mangalam, 2018; Osiurak et al., 2010). This lack of consensus is not surprising if we remind ourselves that consensus on a definition of tool use amounts to consensus on the underlying psychological processes. Regardless, the definitions given by Beck (1980), and updated By Shumaker et al. (2011) are perhaps the most thoroughgoing and influential attempt in this respect, although Beck (1980; also see Hansell \& Ruxton, 2008) warned that his definition only reflects a behavioral description and does not imply any biological or psychological distinctness.. These definitions have two significant merits. The first feature is that these authors did not merely define 'tool use', but also concepts that are very close to tool use, such as tool making and construction (Table 1). Thus, their definition of tool use is also a definition by exclusion (e.g., excluding tool making and construction). The second merit is that their definition is derived from the animal behavior literature, which facilitates discussions of the specificity of tool use to particular species.

What psychological processes are involved in tool use? The emphasis placed by Beck (1980) and Shumaker et al. (2011) on object manipulation is consistent with a vast literature on tool use that has repeatedly stressed the critical role of manipulation-related psychological processes or, more generally, of embodied cognitive processes. However, the embodied cognition approach is not unitary - there are several different versions. The first version is in direct line with the ecological approach to perception and action initially developed by Gibson (Gibson, 1966, 1979), in assuming that tool use can be fully understood as perception-action processes required for effective execution (Biryukova \& Bril, 2012; Bril et al., 2009, 2012; Fragaszy \& Mangalam, 2018; Kahrs \& Lockman, 2014; Lockman, 
2005; Mangalam, 2016; Mangalam \& Fragaszy, 2016; Pagano \& Day, 2020; Smitsman, 1997; 88 Smitsman et al., 2005; Wagman \& Carello, 2003). This perspective pays particular attention to the users exploit this information when manipulating a tool. For instance, as mass increases and the center of mass is located farther from the point of rotation, objects are perceived to be more appropriate for power tasks (e.g., striking a nail or throwing for distance), and as mass decreases and the center of mass is located closer to the point of rotation, objects are perceived to be more appropriate for precision tasks (e.g., striking a small nail or throwing for precision) (Michaels et al., 2007; Wagman et al., 2016; Wagman \& Carello, 2001).

Another version has been developed in parallel, mainly from the neuropsychological literature (Buxbaum, 2001; Heilman et al., 1982; Rothi et al., 1991; van Elk et al., 2014). Contrary to the aforementioned (ecological) perceptuomotor approach, this version assumes that conceptual knowledge is directly extracted from the sensorimotor experience with familiar tools (e.g., hammer, knife). This knowledge about manipulation offers a processing advantage by avoiding the reconstruction de novo of each step of the process. A third approach has been formulated mainly from the idea that tools help modify the physical environment. Here, the emphasis is on the physical (or technical) reasoning involved to understand and perform these modifications (Goldenberg and Spatt, 2009; Johnson-Frey, 2004, 2003; Osiurak et al., 2020b; Vaesen, 2012). This approach can be viewed as 'disembodied,' considering that manipulative aspects are secondary and not the critical component of tool use.

Which of these psychological processes are specific to tool use? As defined by Beck (1980; see Fragaszy \& Mangalam, 2018; Shumaker et al., 2011), tool use is notably characterized by the fact that it implies manipulating an external object to modify the state of the physical environment. However, tool use is not the only behavior that involves both features (i.e., manipulation and physical modifications). Humans and many nonhuman species alike manipulate objects. Tool use concerns not just object-manipulation but also object-object manipulation. This supposes the existence of an attentional shift from the effector on the body (e.g., hand, beak) to that on the tool (e.g., hammer's head, screwdriver's tip) (Fragaszy \& Mangalam, 2018; Mangalam \& Fragaszy, 2016; Osiurak \& Federico, 2020), a phenomenon called "distalization of the end effector" (Arbib et al., 2009). A critical question is whether distalization of the end effector is specific to tool use. Construction behavior, like tool use, also results in modification in the physical environment, raising the question whether the same psychological processes underlie tool use and construction behavior (Arbib, 2012; Walsh et al., 2011). The question of the specificity of psychological processes to tool-use behavior can be extended to other aspects, such as the ability to combine several tool-use actions (meta-tool-use, such as using a tool to acquire another one; Taylor et al., 2007) or to use one tool to create another one.

Which psychological processes involved in tool use are specific to Homo sapiens? Humans, we now know, are not the only species that use tools. Thus, our fascination with tool use behavior extends to other tool-using species in which tool use takes on a different character (Fragaszy \& Mangalam, 2018; Mangalam \& Fragaszy, 2016). Often observation of a member of nonhuman species using a tool prompts discussions about the advanced technical intelligence in that species compared to other, non-tool-using species (Chevalier-Skolnikoff, 1989; Huber and Gajdon, 2006; Mather, 1994; Matsuzawa, 2001; Osiurak and Reynaud, 2020a; Parker and Gibson, 1977; Seed et al., 2009; Vaesen, 2012; van Schaik and Burkart, 2011). This perspective has been criticized (Emery and Clayton, 2009; Haslam, 2013; Wagman et al., 2019), and an outstanding question is whether at least some psychological processes involved in tool use are unique to the human lineage.

This brief overview of these four significant questions that dominate the literature on tool use illustrates the richness of the research topic. However, it also stresses the divergences among the different approaches to addressing these questions, which can be confusing for specialists and nonspecialists alike. A synthesis that confronts divergent approaches is critical to identify productive ways forward to investigate this phenomenon. The present review aims to fill this gap. Its originality lies in including a contribution from scientists from experimental psychology, developmental 
138 psychology, comparative psychology, cognitive neuroscience, cognitive archaeology, and ergonomics, 139 addressing the above questions. A global synthesis will be offered at the end of this review to help 140 orient future research on this fascinating topic. 


\section{Madhur Mangalam \& Dorothy M. Fragaszy}

\section{Overview}

We approach the topic of engagement with tools (henceforth, tooling) from a comparative, evolutionary perspective to explain phylogenetic and developmental origins of these behaviors in diverse species. Unfortunately, in our field, nearly all published works describe observations of nonhuman animals using objects to achieve a goal as 'tool use,' but rarely do they use these observations to test theoretical predictions relevant to the development, form, or effectiveness of the behavior because there has been no theory generating testable hypotheses regarding this behavior.

A theory that supports prospective experimental work is essential for progress in this field. We have been developing an embodied theory of tooling (Fragaszy \& Mangalam, 2018) that integrates concepts from ecological psychology and movement science. Ecological psychology provides the concept of affordances, enabled by the perception of object properties and surface layouts (including spatial relations among objects and orientation of body segments and objects attached to the body as a unit to objects in the environment) through the use of exteroceptive and exproprioceptive information (Gibson, 1966; Shaw, 2001). Movement science provides the concept of controlling the bodily degrees of freedom for functional coordination of movements (Bernstein, 1967; Bernstein et al., 1996) and the concept of situating an activity within the organism-task-environment system (Newell, 1986; Newell and Jordan, 2007). This embodied theory of tooling is equally applicable to humans and other animal species. We use 'to tool' as a verb, meaning to act with an object to achieve a mechanical goal in the service of a functional goal (e.g., hit a nail to drive it into adjoining planks—the mechanical goal-to join the two planks into a rigid unit-the functional goal) and 'tooling' as a noun label for these actions. This wording privileges actions rather than an object, although an object is intrinsic to the activity, just as when we speak of "eating" rather than 'ingesting food' as the general phenomenon.

What constitutes tool use? We propose the following definition derived from our embodied theory: "Tooling is deliberately producing a mechanical effect upon a target object/surface by first grasping an object, thus transforming the body into the body-object system, and then using the body-object system to manage (at least one) spatial relation(s) between a grasped object and a target object/surface, creating a mechanical interface between the two" (Fragaszy \& Mangalam, 2018, p. 194). Here, 'deliberately' implies that tooling is goal-directed. This definition necessitates that the actor, the [grasped or attached-to-the-body] object, and the target come in contact with each other during the activity. That is, tooling begins with the act of establishing a spatial relation between the object attached to the body and the target, and lasts as long as this relation is maintained. For example, when hammering a nail into adjoining planks to make a rigid structure, tooling begins when the actor places the nail against the surface of one plank, continues as the actor strikes the nail with the hammer, and ends when the actor stops striking the nail and switches to some other activity.

This narrow definition of tooling differs from prevalent definitions (e.g., Shumaker et al., 2011) that define tool use as the use of an external object to achieve a goal, with diverse additional conditions appended by various authors (Crain et al., 2013). In our view, many behaviors that have been traditionally described as tool use in nonhuman animals are properly categorized as instrumental problem-solving (acting on/with an object in some way to solve a problem, such as opening a door to pass through a wall), but not as tooling, which is a particular subset of this large class of behaviors (Table 2). A definition of tool use primarily couched in the goal-directed nature of action with an object, which may be adequate when applied to humans because tooling is so pervasive in our behavior, is so ambiguous when applied to nonhuman animals that the topic of tooling in nonhuman animals largely remains a scientific curiosity, outside of theoretically-motivated biological or psychological inquiry. A narrower definition drawn from a particular theory affords analytical clarity and could support 
190 productive engagement of scientists across disciplines. Note that whether a behavior with an object 191 qualifies as tooling or rather as some other form of instrumental problem-solving has no bearing on a 192 judgment about the user's 'intellect' or the complexity of its behavioral/cognitive/neural processes. In 193 fact, all forms of instrumental problem-solving merit investigation of their distinctive characteristics. A 194 classification of action as tooling or not determines the analytical strategy one takes to examine the 195 action; that is all.

196 What psychological processes are involved in tool use? Our theory addresses tooling at the behavioral level. It draws attention to functional task demands (to create a particular form of mechanical interface-e.g., striking a nail at a particular angle with enough force to drive it into the plank), and to movement coordination in meeting these demands (Fragaszy \& Mangalam, 2018). It further draws our attention to the perceptuomotor processes supporting relational actions (moving a grasped object in relation to another object or surface) necessary to meet functional task demands. In accord with our ecological stance (Gibson, 1966; Harrison \& Stergiou, 2015; Wagman \& Miller, 2003), we do not distinguish between perception and cognition. Perceptuomotor processes relating to (a) perceiving spatial relations among objects and surfaces, (b) developing agency over objects attached to the body (the distalization of the end effector), and (c) controlling the bodily degrees of freedom to meet functional task demands characterize tooling. These processes occur in the unified body-objecttask-environment system; that is, tooling is an action of a given individual with specific materials to accomplish a particular task in a particular setting (Bril et al., 2012; Mangalam \& Fragaszy, 2016; Smitsman, 1997).

We suggest joint deployment of these three perceptuomotor processes distinguishes tooling from other instrumental problem-solving. Particular experiences accompany these processes in humans. We do not know if nonhuman animals experience tooling as humans do, but these same perceptuomotor processes must occur in some form.

- $\quad$ Establishing and managing spatial relation(s) between an object attached to the body and a target object or surface. Tooling requires establishing and managing at least one spatial relation between an object attached to the body and a target object or surface (see Fragaszy \& Mangalam, 2018, for detailed explanation). Spatial relations in a tooling activity can vary in several dimensions. First, the number of relations can vary; tooling can involve managing more than one spatial relation between the body, the tool, and the target object or surface, sequentially, or concurrently. For instance, to join two planks, an individual might align the planks in a certain way (first spatial relation), then place a nail at a particular point on the top blank (second spatial relation), then strike the nail with the hammer (third spatial relation) to drive it through the planks. Spatial relations can differ in their specificity: placement, orientation, and geometric alignment. Consider, for example, using a screwdriver to drive in a screw. The screwdriver's distal end must be placed quite precisely on the screw's head, and the screwdriver's shaft must be oriented parallel to the screw's long axis to enable the screwdriver's head to fit into the screw's head. Spatial relations can also vary in temporal dynamics, from static to dynamic. The relation between the two planks in the previous example is static because once they are placed in position, they are not moved relative to each other. Similarly, the nail is placed in a fixed location on the top plank. The relation between the hammer and the nail is dynamic because the hammer is moved relative to the nail.

- Distalization of the end-effector. Tooling requires the distalization of the end-effector-the locus of perceptuomotor control-from the body to the part on the tool that acts upon the target object or surface (Arbib et al., 2009). Studies have shown that in monkeys using pliers to grasp a food item, the grasp-related cortical regions encode for movements of the pliers' jaws rather than movements of the fingers (Umiltà et al., 2008). A specific grip configuration may be imposed by the mass, shape, and size of the pliers, and movements are adapted to the pliers' jointed feature (direct vs. reverse pliers). These findings have been replicated in humans (Gallivan et al., 2013). Tooling is also associated with the remapping of the space surrounding the body to perceive reaching affordances from the frame of reference of the handheld tool rather than the hand (Berti and Frassinetti, 2000; Farnè et al., 2005; 
Maravita and Iriki, 2004), and remapping of somatic senses to perceive what is happening at the tool 241 tip rather than at the fingertips (Miller et al., 2018; Takahashi \& Watt, 2017).

- $\quad$ Coordinating the body-object system. The body-object system is coordinated differently than the body-only system, as manifest in different movements and postures. A simple example makes this point: different postures and movements are used when gripping a nail with the hand than when gripping it with pliers. Even when the task demands do not manifestly require different postures and movements, moving with a grasped object alters movement coordination. For example, people move their arms differently to stabilize the end-point trajectory when using the arm and hand to point at a target than when pointing at the same target with a stick (Valk et al., 2016; van der Steen and Bongers, 2011).

Which of these psychological processes are specific to tool use? None of the above perceptuomotor processes is specific to tooling. The acts of establishing and managing spatial relation(s) between an object attached to the body and a target object or surface are part of several non-tooling activities and are observed in both humans and nonhuman species, such as chimpanzees and capuchin monkeys aligning a stick to a matching cut-out in an experimental task (Fragaszy et al., 2011, 2015; la Cour et al., 2014), or a weaver bird inserting grass or twigs into a partially completed nest (Walsh et al., 2011). The end-effector is distalized in many non-tooling contexts, such as when using a manipulandum to move a cursor on the screen, although this behavior has been classified by some as tool use (Heald et al., 2018; Ingram et al., 2010). (Using a manipulandum to move a cursor does not qualify as tooling according to our definition because the manipulandum does not directly and mechanically affect the screen.) We coordinate the body-object system all the time in non-tooling contexts, such as when passing through a doorway sideways when holding an object wider than the doorway (Higuchi et al., 2006), or when using assistive devices-from wheelchair to exoskeleton (Pazzaglia and Molinari, 2016). Transporting an object inevitably involves coordinating the body-object system, irrespective of the context. These examples clearly illustrate that each perceptuomotor process is involved in many activities beyond tooling.

Which psychological processes involved in tool use are specific to Homo sapiens? We do not suppose that tooling is specific to humans in a holistic way, although we have no doubt that there are large differences among species in the elaboration and efficiency of each of the three perceptuomotor processes listed above. Unfortunately, few comparative studies isolate each one of the three processes. The few we have suggest deep differences across species in two of the three processes but some commonality between humans and other species in the third. With respect to perceiving and managing spatial relations between a grasped object and another object, by two years of age, humans readily align a straight stick to a matching cutout in a platform surface, whereas adult chimpanzees and capuchin monkeys do so imprecisely (Fig. 1) (Fragaszy et al., 2011, 2015; la Cour et al., 2014), suggesting a deep difference between humans and nonhuman primates in this domain. With respect to coordinating the body-object system when tooling, some evidence indicates that nonhuman species can do this modestly, but not as effectively as humans. For example, when cracking nuts using stone hammers, capuchin monkeys alter their strikes to strike a nut with less or more force based on the type and condition of the nut (Mangalam et al., 2016; Mangalam \& Fragaszy, 2015); they do so by adjusting the amplitude [and velocity] of the strike but do not adjust the hammer's kinetic energy at impact (Fig. 2) (Mangalam, Pacheco, et al., 2018), as do humans (Mangalam et al., 2020; also see Bril et al., 2012). Humans can control a larger number of functional parameters of the task when hammering with stones, as when manufacturing sharp flakes from stone cores, than nonhuman primates using stone hammers (Bril et al., 2015, 2012). With respect to distalization of the endeffector, Umiltà et al.'s (2008) work with macaques using pliers, mentioned above, stands as one example suggesting that nonhuman species, like humans, do distalize the end-effector when tooling to meet task demands.

What must be the direction for future work on tool use? We suggest that expanding our understanding of each of the three component perceptuomotor processes we have highlighted above, and our understanding of their integration in tooling and other instrumental actions with objects, will be 
particularly useful. Ethologists may most naturally consider these processes in diverse species and developmental, ecological, and evolutionary perspectives. Psychologists may most naturally consider them in relation to attentional and related cognitive demands, particularly affordance learning and skill learning. Neuroscientists may most naturally consider them in relation to the organization and function of the nervous system. One obvious question for neuroscientists is to identify which tooling actions are coordinated differently than achieving the same goal with the unaugmented hand. Findings from studies on reach-to-grasp movements using the hand or using the hand with a tool (e.g., grasping with fingers or with a tong) suggest an effector-independent neural encoding of movements (Gallivan et al., 2013, 2011; Umiltà et al., 2008). The interpretational nuances added by comparing actions with different effectors to achieve the same goal would yield a more reliable neurophysiological understanding of tooling than the currently available explanations based on comparisons of actual tooling with gesturing with an object (Hermsdörfer et al., 2012, 2007).

Gesturing with an object (pantomiming) is the principal paradigm used to investigate the neural basis of tooling particularly in relation to cognitive and sensorimotor deficits in neurological patients (Buxbaum et al., 2005; Króliczak and Frey, 2009; Martin et al., 2016b). A plethora of interesting findings of how brain-damaged individuals move objects gesturally paint an optimistic picture of what we understand about the neural correlates of tooling (Goldenberg and Spatt, 2009; Johnson-Frey, 2004; Renfrew et al., 2008). However, pantomiming with objects is not comparable to tooling in any biologically relevant way (a detailed discussion is beyond the scope of this essay; we hope to address this issue in greater detail in a future piece). Behavioral neuroscientists could investigate tooling more productively if they set aside the notion that tooling is an index of 'complexity' to focus on the distinctive perceptuomotor processes of tooling noted above.

Bernstein et al. $(1967 ; 1996)$ stressed the importance of the senses to the coordination of actions. From a different theoretical orientation, Gibson (Gibson, 1966) arrived at a convergent conclusion. There is an untapped opportunity to study how the sensorimotor apparatus of species/individuals constrains their engagement in specific forms of tooling (Martinho et al., 2014; Troscianko et al., 2012). Accounting for the anatomy, physiology, and sensory processes of the user is critical to situate 'tooling' within the realm of biological inquiry. 


\title{
Tool use as detection and exploitation of information in an ecological niche
}

\author{
Jeffrey B. Wagman \& Brian M. Day
}

\section{Overview}

In the ecological approach to perception-action, the relationship between animal and environment lawfully structures pattered energy arrays so as to provide information about this relationship. The lawful structuring of energy distributions entails that information about a given affordance is invariant over transformations including whether or not a tool alters this fit. Therefore, perceiving and actualizing affordances for performing a goal-directed behavior with or by means of a tool is the detection (and exploitation) of information-no more and no less. Moreover, any animal species capable of detecting and exploiting such information is capable tool use. Although tool use does not seem to be dependent on the sophistication of particular cognitive apparatus, it may be dependent on the sophistication of a given niche. Consequently, tools that are unique to the human niche (such as technological interfaces or virtual tools) are an important area for future research.

What constitutes tool use? The ecological approach to perceiving and acting focuses on a scientific understanding of the lawful perceptual control of everyday goal-directed behavior. In this approach, the fundamental unit of analysis is the animal-environment system, not the animal or the environment in isolation (Gibson, 1979; Turvey, 2018). In the ecological approach, it is (only) at this level that all psychological processes-including tool use-occur. A key reason for this focus is that the relationship (i.e., the fit) between the animal and environment determines what possibilities for behaviors-what affordances- are available to that animal (Wagman, 2020). It is also at this level that these possibilities become actualized in the performance of a given behavior.

A tool alters the fit between animal and environment by changing its ability to perceive or actualize affordances. Therefore, tool use is the exploitation of this altered animalenvironment fit in the context of performing a given goal-directed behavior. By this definition, using a hammer to drive a nail qualifies as tool use. The hammer changes the ability to actualize many affordances, including the ability to forcefully strike another object. It is this change that is exploited in achieving the goal.

Alternatively, merely carrying a hammer does not qualify as tool use. This is tool transport rather than tool use. Stacking stones to build a barrier also does not qualify as tool use. This is construction behavior rather than tool use. In both cases, affordances are actualized in the context of a goal-directed behavior. However, in neither case does the object (the hammer or the stones) change the animal's ability to perceive or actualize affordances. Actualizing affordances is not the same as tool use. Bending a straight wire into a hook also does not qualify as tool use. Before and after it is bent, the wire alters the fit between animal and environment. Though bending the wire is likely to be goaldirected, doing so does not-in and of itself-exploit how it alters the fit between animal and environment. Changing the affordances of a given object is not the same as tool use. Instead, this is tool making or tool modification.

However, throwing stones at an aggressor, using a wheelbarrow to transport stones, and using a bent wire to retrieve a food item each qualify as tool use. Similarly, using a flashlight, a long cane, or a guide dog to safely navigate a cluttered environment also qualify as tool use. In each case, these objects change the ability to perceive and actualize affordances in ways that are exploited in achieving the goal. 
What psychological processes are involved in tool use? In the ecological approach to perceiving and acting, the relationship between animal and environment lawfully structures patterned energy arrays. The structure actively encountered at a point of observation in such arrays provides information about this relationship (Fig. 3, bottom). That is, it provides information about affordanceswhether, when, and how to move to achieve a given goal. Thus, perceiving affordances for performing a goal-directed behavior is the detection (and exploitation) of such information-no more and no less (Wagman et al., 2019).

When the fit between animal and environment is altered by means of a tool, the structure encountered at a point of observation provides information about this altered relationship (see Fig. 3, middle). That is, it provides information about affordances for tool use-whether, when, and how to use the tool to achieve a given goal. Consequently, perceiving and actualizing affordances for performing a goal-directed behavior with or by means of a tool is also the detection (and exploitation) of information-no more and no less.

The lawful structuring of energy distributions entails that information about a given affordance is invariant over transformations, including the particulars of the energy being structured and the anatomical component(s) used to detect such structure. This invariance over transformation is readily demonstrated in a context common to tool use-the hefting, wielding, or manipulating of objects by muscular effort (see Carello \& Turvey, 2016). When wielding an occluded object, people can perceive many different affordances of that object, and they can perceive a given affordance of that object under many different wielding circumstances (Hajnal et al., 2007; Mangalam et al., 2017; Wagman et al., 2017). By the same token, when people probe a surface with an object, they can perceive many different affordances of that surface, and they can perceive a given affordance of that surface under many different probing circumstances (Wagman and Hajnal, 2016, 2014a, 2014b).

Perceiving affordances of, and by means of, a tool requires spontaneously and temporarily assembling task-specific detection units from potentially independent components (across both body and tool). Actualizing affordances of a tool requires an analogous process of spontaneously and temporarily assembling task-specific control units from potentially independent components (again, across both body and tool). The detection and exploitation of lawfully structured energy arrays likely underwrites both phenomena (Carello and Wagman, 2009; Profeta et al., 2020).

Which of these psychological processes are specific to tool use? As described in Section 2, in the ecological approach, lawfully structured patterned energy arrays provide information about whether, when, and how to move to achieve a goal. Such information is invariant not only across transformations of the energy media and anatomical components but also whether or not an animal exploits an altered animal-environment fit in the context of achieving the goal (i.e., whether or not an animal engages in tool use).

When an animal moves from place to place in a cluttered environment, it encounters a lawfully generated pattern of optical structure that is informative about the animal-environment relationshiphow, when, and where it is moving. Moreover, such patterns are informative about whether, when, and how a person must move to achieve a goal (e.g., catching a ball, see Fink et al., 2009). Critically, such patterns are also informative as to whether, when, and how a person must move a tool such as a vehicle to achieve a goal (e.g., steering or stopping safely, Fajen, 2013; Fajen, 2007; Fajen \& Matthis, 2011; Kadar \& Shaw, 2000).

Likewise, when an animal moves one of its limbs about a given joint, it encounters a lawfully generated pattern of resistance to rotational acceleration that is informative about the relationship between animal-environment relationship-how, when, and where the limb is moving. Moreover, such patterns are informative about whether, when, and how a person must move that limb to achieve a goal (e.g., pointing at a target, Pagano \& Turvey, 1998). Critically, such patterns are also informative as to whether, when, and how a person should move a hand-held tool to achieve a goal (e.g., hammering or displacing another object (Wagman et al., 2016; Wagman and Carello, 2001). 
In both cases, it is irrelevant whether achieving the goal requires controlling the movements of the person or the person-plus-object system. The lawful structuring of patterned energy arrays by the relationship between animal and environment entails that the information about how, where, and when to move to achieve the goal is invariant across this transformation (see Fig. 3, middle). This likely explains why tools, be they vehicles or hand-held objects, are often 'functionally transparent' to skilled users. In both cases, tools are perceived as part of the body because they are perceived in the same way as the body (Pagano and Turvey, 1998).

Which psychological processes involved in tool use are specific to Homo sapiens? The lawful structuring of energy distributions by the relationship between animal and environment entails that information about a given affordance is invariant across the sensory apparatus, nervous system, and brain of the animal doing the perceiving. Such details are irrelevant so long as that animal can detect the information about whether, when, and how to move to achieve a given goal. Incontrovertibly, animals across all phyla perceive and actualize affordances. According to the ecological approach, these abilities result from the same lawful processes across species (Turvey, 2018; Wagman et al., 2019). Moreover, the abilities of animals to exploit how a tool alters the ability to perceive and actualize affordances are also the result of the same lawful processes across species.

Tool transport, construction behavior, and tool modification are widespread across the animal kingdom - even in so-called 'lower animals' such as crustaceans, insects, worms, and amoeba (Turvey, 2018; Wagman et al., 2019). Instances of tool use (as defined above in section 1), however, are less common-occurring mostly (but not exclusively) in birds and primates (Hunt et al., 2013). This observation begs the question as to why instances of tool use are not more common across the animal kingdom - especially if, as we suggest, the information about a given affordance is invariant across sensory apparatus, nervous system, and brain.

We propose that this disparity is not due to differences in neural machinery (or intelligence) across species. Rather, we propose that it is due to differences in the ecological niche occupied by those species. A niche is how a particular species lives, given the fit between animal and environment particular to that species. A niche is a way of life-a set of affordances for-a particular species (Gibson, 1979; Turvey, 2018). Species occupying different niches necessarily encounter (and perceive and exploit) different affordances. The more complex the niche of a given species, the more diverse and complex the set of affordances available to that species' members, and the more likely those members will (learn to) perceive and exploit affordances for tool use.

Accordingly, species other than primates and birds engage in tool use when their niche is modified to include the opportunity (to learn) to perceive and exploit these affordances. For example, rats perceive and exploit affordances for driving a vehicle when their niche is modified to include opportunities to (learn to) do so (Crawford et al., 2020). Moreover, the driving skills of rats raised in an enriched environment (i.e., an enriched niche) are superior to those raised in a standard environment.

What must be the direction for future work on tool use? Affordances (for tool use) are relative to the niche that an animal species occupies. Humans occupy a unique niche that includes a vast array of technological, communication, and representation systems. In this niche, affordances for tool use include teleoperation of the movements of search and rescue robots, the Mars rover, or surveillance drones; the manipulation and repair of internal bodily organs and tissue by means of laparoscopic surgerical instruments; and even the (un)locking of the door to one's home using a smart phone app. In all such cases, these devices change the ability to perceive and actualize affordances in ways that are exploited in achieving the goal.

In the general case, information about affordances for tool use is available in the structure in patterned energy encountered at a point of observation. However, in the situations described above, information about affordances is available only by means of an interface (e.g., a computer-mediated visual display). In these cases, preserving the lawful structuring of information about affordances requires designing the interface to preserve, enhance, or (in some cases) even generate such lawfulness (Pagano \& Day, 2020; Fig. 3, top). Designing interfaces that allow for the perceiving 

- no more and no less-is an essential area for future research.

Along these lines, researchers programmed a custom laparoscopic surgery simulator to provide (haptic) information about the extent to which (simulated) bodily tissue could be manipulated until it was (accidentally) torn (Altenhoff et al., 2017; Hartman et al., 2016; Long et al., 2016). They modeled haptic information about 'distance to break' on optical information about 'time to contact' with a surface. They found that both novice participants and experienced surgeons improved their 471 performance in this task with only minimal practice. That only minimal practice was required 472 (regardless of expertise) is impressive. However, it also demonstrates a fundamental fact about perception of affordances with or without a tool or interface. Namely, the mere availability of information about whether, when, and how a person must move to achieve a goal does not entail that such information is detected or exploited. Therefore, investigating the necessary and sufficient experiences to facilitate the functional transparency of a virtual tool or a user-tool interface (Day et al., 2019, 2017) is another important area for future research. 


\section{Tools as intermittent properties of the fractal coastlines between organism and environment}

Damian G. Kelty-Stephen

\section{Overview}

Tool use rests on the cascades underwriting organism-and-environment relationships. Cascade dynamics comprise nonlinear interactions across nested scales of activity. These nonlinear interactions across scales support scale-free flexibility resulting in an organism with porous and blurry boundaries. Organisms spread fluidly over contextual constraints, contacting the environment along intermittent coastlines where behavior ebbs and flows against the more stable land. The tool-using organism can be more adaptive as it is more intermittent, avoiding locking in, extending and retracting, wandering, and hovering. Tool use is just another intermittent function in the daily work of an organism, for example, picking up a tool, setting it aside. However, the more startling possibility is that tools are themselves intermittent participants in the organism-environment exchanges.

Tools are necessarily tuned to the scale of a task. So, their intermittency may require viewing Gibsonian ecological psychology through Mandelbrot's multifractal-geometrical lens. For Gibson (1979), organisms and the environment entail one another, and any coastline between them is porous and blurry. When studying the coastlines bounding Britain, Mandelbrot (1967) realized that such boundaries embody fractional (or 'fractal' for short) dimensions. Off-putting at first glance, fractal dimensionality aims to do the important job of quantifying structure that fails to be integer-dimensional (e.g., zero-, one-, two-, or three-dimensional points, lines, surfaces, or Euclidean solids). Coastlines with varying fractionality can crucially originate from nonlinear interactions across scales. Indeed, the nonlinear interactions across scales-that is, the cascades-entail variation in these fractal dimensions. That is, they entail 'multifractal' dimensionality (Mandelbrot, 2013).

The multifractal structure is thus a meaningful way to understand and model tool use. Organisms' exploration of tools and task context depends on multifractal fluctuations in organisms' movement (Doyon et al., 2019; Hajnal et al., 2018; Kelty-Stephen \& Dixon, 2014; Mangalam et al., 2019; Mangalam, Carver, et al., 2020a; Mangalam, Chen, et al., 2020; Mangalam, Carver, et al., 2020b; Mangalam \& Kelty-Stephen, 2020; Stephen et al., 2009, 2010; Stephen \& Hajnal, 2011). This insight may lead to the startling idea that that tools could be intermittent, blinking in and out of existence as a separate entity from the body. In this sense, the muddiness of 'tool' concepts noted by Fragaszy and Mangalam (2018) may be a defining feature rather than muddiness in the scholarship.

What constitutes tool use? An organism uses a tool when it takes hold of an object to engage in direct mechanical interaction with a target surface (Fragaszy \& Mangalam, 2018). However, the scalefree aspect of cascade dynamics suggests that tool use should extend beyond the constraints of this definition.

Tool use involves a mutual contingency between tool and user, not simply addition entailed by Fragaszy and Mangalam's (2018) 'body-plus-object' system. Specifically, tools inherit multifractal fluctuations from manipulating limbs, and tools pay back a dividend of new multifractal fluctuations to limbs. This point is borne out if we examine the manual exploration of a handheld tool. We can use vector autoregression to model specific dyadic relationships among multifractality estimates on the tool, the hand, the wrist, the forearm, and the elbow (Mangalam, Carver, et al., 2020a, 2020b).

Because the organism-environment relationship is never adrift from long-scale contingencies, it is not crucial that the tool and skin remain in constant immediate contact. Fragaszy and Mangalam (2018) stress the need for immediate contact with grasping skin or target surface, but cascade-driven 
interactions allow no straightforward segregation of short-range interaction from more distal forces. Rather than an empty expanse, environmental space is full of clutter whose integral flow supports organisms via contact through distributed-but-rarely-distinct modalities (e.g., optical, acoustic, and haptic (1979), Stoffregen \& Pittenger (1995), and Harrison et al. (2021), respectively). The specificity of localized sensor cells is physiologically real, but they distract from the non-exclusivity and often mutuality of manipulation and, say, observation when mechanical contact is lost (Stoffregen et al., 2017). Cascades span cross-scale interactions through these ecological flows like a spiderweb of contingency that the organism then manipulates to extend its reach. Even if a tool drops from reach or hurtles towards a visible target, the looking for or after the tool is no less mechanical even if the skin contact is lost.

What psychological processes are involved in tool use? The processes involved are cascades, that is, physical processes that branch, fracture, avalanche, diffuse, or otherwise spread apart across multiple scales across space or across time. Organism physiology exhibit cascades spatially (e.g., hierarchical branching of neural dendrites, cardiovascular vessels, and capillaries (Goldberger et al., 1990), actin-myosin architectures (Fernandez-Gonzalez and Zallen, 2011), DNA supporting cells (Dragovich and Mišić, 2019), collagen composing bones (Fratzl, 2008), and tensegrity-like networks of connective tissue that span the entire organism, wrapped tautly across the muscles and bones (Turvey and Fonseca, 2014)) as well as through a correspondingly hierarchical temporal structure whenever these multifractally-shaped parts operate (e.g., neuronal avalanches (Zorick et al., 2020). The spreading interactions across multiple scales of this hierarchy (e.g., Ingber, 2006) entail a multifractal structure (Turvey and Fonseca, 2014).

The cascades pervade the organism-environment system-not just through neurons but through musculoskeletal systems and connective tissue supporting manipulation and observation alike. The nesting within an organism, within the environment, of the organism within the environment does not leave any other alternative. The only issue is how these nestings change as events trigger new cross-scale relationships. Multifractal fluctuations appears to predict this cascade-driven coordination of events-within a single organism (Carver \& Kelty-Stephen, 2017; Mangalam, Carver, et al., 2020b), between organisms (Carver and Kelty-Stephen, 2017), and between organism and environment (Stephen and Dixon, 2011; Teng et al., 2016)—no matter whether the coordination is visual or mechanical.

Which of these psychological processes are specific to tool use? These processes are not specific to tool use. Cascading fluctuations support organism-organism links as well as organismenvironment links. For instance, coordination within honey-bee colonies correlates with cascade dynamics: multifractality of individual-bee activity is an effective classifier of colony membership (Carver and Kelty-Stephen, 2017). Organisms are not tools, except in cases of social dominance or parasitism. The agency of an organism contrasts clearly with the passivity of the tool. The only potential ambiguity worth focusing our attention on is the blurring of the distinction between tool and organism, for example, when tools become part of the bodies or when body parts can serve as tools.

Which psychological processes involved in tool use are specific to Homo sapiens? The capacity to manipulate environmental materials as tools to further goals belongs to more species than humans. For instance, an amoeboid slime mold (Dictyostelium discoideum) uses the molecule cyclic adenosine monophosphate (cAMP) to navigate a ground surface to find and coordinate with other conspecific amoebae. $D$. discodeum spend the earlier, better-fed days as an amoeba, and it is only as food becomes more scarce that the amoebae coalesce into a multicellular body-either a fruiting body or a slug for locomotion to a niche with better nutrient stores (e.g., Schaap, 2021). This drama has all of the necessary features of tool use, but it also shows how the distinctions between tool and user may blur.

In the traditional sense, cAMP fits all the needs of a mechanical tool. The amoebae are manipulating cAMP, grasping molecules with its cell-membrane receptors, using the molecules to orient their cytoskeletal posture towards and to inch up the cAMP gradient to the source (Chisholm and Firtel, 2004). Eventually, individual amoebae on the move will use cAMP to attract each other 
along the way, using a cAMP relay to latch on to slow amoeba behind them (Bagorda et al., 2006). Hence, at all points, the amoeba grasps cAMP and manipulates it to operate on the ground surface and the surface of other cell membranes.

The organismic coastlines of this example blur in three significant respects. First, the tool is not so clearly separate from the body. The cAMP is a molecule that amoeboid slime molds secrete themselves in the first place. This chemical attractant is the stress response of a hungry amoeba that has run out of food. So, if one amoeba can grasp cAMP, it means that another amoeba has run out of food. Moreover, the relay use of CAMP involves the amoeba producing its new CAMP (Chisholm and Firtel, 2004). Second, this tool essentially invents a new body, dramatically redrawing the coastline between organism and environment. All of the cAMP-chasing culminates in amoebae aggregating to form a multi-cellular fruiting body that will disseminate the next generation of amoeboid slime-mold spores. The aggregate can also take the intermediary form of a slug that can sense light and heat to navigate a more conducive niche. The multi-cellular aggregates are richly differentiated but integrated whole: the aggregate prompts once-uniform amoeba to take on specialized forms and functions to serve the goals of the whole slime-mold colony (Bagorda et al., 2006). The multi-cellular body is no less real or integral than the amoeboid one had been. Furthermore, cAMP flows within just the same, regulating the now tighter-knit coordinations of many no-longer-amoebae. In the first blurring, a castoff product of one body had become another body's tool. In this second blurring, the tool vanishes, receding within the newly redrawn coastline of the organism. Indeed, the tool has left the biological theorist befuddled (e.g., Wilson, 2000). How many organisms are there? One? Hundreds? The tool for hundreds of bodies has become the endogenous fuel for the one larger body that remains.

The third sense in which the organism-tool coastline blurs is that the body can fashion tools from itself. This larger multicellular body builds itself a form that features tool-like extensions. The differentiation process produces a rounded tip at the head of the slug or fruiting body. The slug can navigate towards light and heat. There is no internal body part, no protein or molecule to do this sensing. No, it is the organism's mechanics: it is the tip's external curvature that focuses on visible and infrared light just like a lens. Hence, the body shapes its degrees of freedom into a tool allowing new manipulation and mechanical contact. The rounded tip focuses visible and infrared light in a way that stimulates metabolic processes to produce more ammonia, which inhibits internal cAMP production at the back or bottom of the slug or fruiting body. Interrupting the cAMP leaves the back or bottom end of the slug or fruiting body slack, leaving the cAMP-full tip to drag it forward (Schaap, 2021). Furthermore, although the tip touches no surface, the light remains no less patterned by the ambient surfaces than in Gibson's (1979). As ever, observation is still mechanical amidst the ecological cascades.

Again, do we need this separate species to make the case that tool use is not specifically human? After all, human bodies are porous and flexible enough. We can fashion tools out of our bodily degrees of freedom, for example, fingertips to dig or eyes, faces, or fingerprints that once scanned can open locks. We also make our tools part of our body when we learn to use prosthetic limbs or teeth. One of the competing theories of the evolution of our own camera-like eyes begins with the possibility that billions of years ago, non-algal organisms learned to subsume photosynthesizing algae (Gavelis et al., 2017; Hayakawa et al., 2015; Lundberg et al., 2012). Algal DNA—that is, no organism but a tool made of (Kianianmomeni and Hallmann, 2014), offering even humans an alternative to retinal prosthesis (Wood et al., 2019). So, our very own eyes need not always have been our own. Our bodies themselves are a patchwork of environmental detritus, emerging over long time scales in a way that might sometimes support adaptive behaviors in the short term.

What must be the direction for future work on tool use? The intermittency of tools points immediately to the question of agency and its bounds. How do organisms extend or retract their scope of influence? How do they push their coastline outwards? Or give ground back to the environment? As the tool shimmers in and out of existence, the organism concedes and engulfs more of its surroundings, respectively. This intermittency of tools is nothing but the negative image of 
626 intermittency of control. An important focus for future study is whether the multifractal geometry of tool 627 use can formalize a cascade-driven notion of agency or control. 


\section{A tool in use is incorporated into synergies}

\section{Raoul M. Bongers}

\section{Overview}

The current contribution presents the first steps of an ecological-dynamical approach to tool use. It is argued that tools are integrated in synergies and that properties of the tool determine the effectivities by which to scale the affordances in the environment. In line with an ecological-dynamical account these processes are not specific for tool use nor for any given species. Some future routes are presented to expand these first steps to a full-blown account of tool use.

What constitutes tool use? During our daily life activities, we often grasp an object in the environment to use that object as a tool to reach our goal. For instance, we use a knife to cut bread and a spoon to stir our coffee. Hence, a tool can be casually defined as an object in the environment that is temporarily attached to the body to act on other objects in the environment to reach a goal (cf. Beck, 1980; Fragaszy \& Mangalam, 2018). Most of the time objects are used as tools when our own action system falls short or when action goals can be achieved more conveniently when performed with a tool, that is, tools alter our capacity for action. Therefore, we take an action-perception perspective on tool use, one of the main lines of research on tool use that are outlined by Fragaszy \& Mangalam (2018). In the current contribution we take this perspective as a starting point to give an outline of how to rigorously apply the ecological-dynamical approach (cf. Profeta \& Turvey, 2018) to tool use.

What psychological processes are involved in tool use? The starting point of an Ecological approach to action-perception is that control of an action does not come from an internal structure (such as a part of the central nervous system), but that coordination of action and perception takes place in an organism-environment system (Gibson, 1979; see Michaels \& Carello, 2000; Richardson et al., 2008; Warren, 2005 for overview papers of this approach). Properties of the elements making up the organism-environment system, together with physical law constrain the myriad of action possibilities to result in goal-directed behavior. An organism moving through an environment creates perceptual flow fields and these flow fields contain invariants that specify properties of an environmental feature or the relation between the organism and the environment. That is, the perceptual flow fields specify functional relations between an organism and its environment that have meaning for an organism and this meaning is perceived directly. Therefore, organisms directly perceive affordances, the possibilities for action in the environment. For instance, someone can perceive whether the size of a handle affords a tool to be graspable with one hand.

Important for understanding tool use is that affordances have their counterparts in the organism, which are dubbed effectivities (cf. Michaels \& Carello, 2000; Shaw et al., 1995). Turvey (1992) argued that an affordance is a dispositional property of the environment that requires a complementary dispositional property in the organism. Both dispositions are necessary conditions for an organism-environment system to actualize an action. For instance, in his seminal paper on affordance perception, Warren (1984) demonstrated that the climbability of a stairs as well as the perception of the climbability (i.e., the affordance) both depend on a body-scaled measure of riser height. Hence, properties of the environment (i.e., affordances) are perceived in terms of the properties of the organism (i.e., effectivities) and an action can only be performed if there is a fit between both properties.

Effectivities are determined by the synergies that can emerge in the action system. From a dynamical systems perspective to movement coordination, synergies emerge from task, organism and environmental constraints in a self-organizing manner. Synergies are defined as the temporary 
676

677

678

679

680

681

682

683

684

685

686

687

688

689

690

691

692

693

694

695

696

697

698

699

700

701

702

703

704

705

706

707

708

709

710

711

712

713

714

715

716

717

718

719

720

721

722

723

724

725

726

727 functional units in which the abundant degrees of freedom are organized (Kelso, 2009; Turvey, 1990, 2007). Synergies maintain stability of its organization through co-variation of the degrees of freedom that make up the synergy (Kelso et al., 1984; Latash et al., 2007; Riley et al., 2011; Schöner, 1995). For instance, when pistol shooting the joint angles in the arm co-vary such that the aiming at the target is stabilized (Scholz et al., 2000). In sum, combining the principles presented in the foregoing gives an account of action-perception in which perceptual invariants act as constraints on the formation of synergies thereby affecting the emergent coordinative pattern in the degrees of freedom in the body in such a way that they are organized in a way to actualize the affordance specified by the perceptual invariants.

An object in the environment that is attached to the body to function as a tool changes the geometry of the action system. For instance, a tool is often held in the hand and thereby the tool changes the shape (i.e., length) of the action system. Equally important, a handheld tool produces forces and torques in the muscles and joints that affect the constraints on the basis of which synergies in the action system emerge. For instance, a tool has a certain weight, and this weight changes the interactions between task, organism and environmental constraints on the basis of which synergies emerge. Hence, a tool in use affects the geometry of the action system as well as the available synergies (Fig. 4). Both these changes affect the effectivities that function as a scale to which the properties of the environment are perceived. That is, a tool in use becomes a part of the action system that establishes the effectivities and therefore the affordances (Bongers, 2001; Bongers et al., 2003).

The incorporation of the tool in the action system has been suggested in different studies examining the kinematic characteristics of the tooling end-effector. When learning to use a complex tool, kinematic characteristics that normally show up in hand movements can also be observed in movements of the tool tip (Heuer and Sülzenbrück, 2009). Moreover, Jacobs et al. (2009) showed that differences in kinematics of the end-effector for tool and non-tool conditions were more pronounced for apraxic patients than for non-apraxic people. In examining the mechanisms underlying these phenomena we analyzed the synergy in the arm during pointing with a rod to a target. Using an Uncontrolled Manifold analysis (Scholz and Schöner, 1999; Schöner, 1995), we showed that there is more co-variation in joint angles stabilizing the position of the rod's tip in space than that there is other variability in the joint angles de-stabilizing the rod's tip position (Valk et al., 2016; van der Steen and Bongers, 2011). Moreover, this effect did not depend on the length of the rod $(10-40 \mathrm{~cm})$ used to reach to a target. In a similar vein, Rein et al. (2013) showed that stone-knappers had more co-variation in their joint angles than variation affecting the position of the hand and Mangalam et al. (2018) showed that bearded capuchin monkeys stabilize the path of the hammer when cracking nuts. Therefore, several studies showed that joint angles co-vary to stabilize the end-effector at the tool. This implies that the tool is incorporated in the elements making up the action system that relate to each other in a synergistic manner. Hence, the tool is integrated in the synergy emerging in the action system consisting of body plus tool.

The incorporation of the tool in the synergy in the arm implies that constraints of the tool interact with constraints of the task, organism and environment to determine the effectivities. A change in effectivities as a function of the properties of the tool in use does also result in a change in affordances (Fig. 4). That is, the environment is perceived with reference to the properties of the action system that consists of the body and the tool. To examine this, several experiments were conducted in which participants had to select a distance from which to displace an object with the tip of a hand held rod (Bongers et al., 2004, 2003). Importantly, the to-be-displaced object was placed at hip-height and it was approached, and the distance had to be selected, with the tip of the rod pointing upwards so that the perceived affordance could be studied. Findings demonstrate that the selected distance was a function of the length of the rod and of the dynamic forces and torques created by the rod. Importantly, these effects interacted with properties of the to be displaced object (Bongers et al., 2004) emphasizing that tooling affordances refer to the relation between the properties of the action system, including the tool, and properties of the environment. The idea that tools affect the affordances and that the specifics of the tooling affordances are perceived by the organism is supported by studies of Wagman and colleagues. For instance, Wagman \& Taylor (2005) showed that affordances can be 
perceived for the tool and for the body+tool system. Moreover, Wagman et al. (2016) showed that affordances of tools are perceived that are assembled out of other objects while also the affordances of the individual objects making up the tool can be perceived.

From the perspective outlined here we define a tool as an object from the environment that is incorporated in the emergent synergies in the action system to perform a goal-directed action. Based on this integration of the tool in the action system the end-effector displaces from the body to the tool and affects the effectivities and thus the affordances. Therefore, tools allow for actions that are not possible without the tool. Effectivities and affordances follow from interactions between constraints in the organism-environment system and physical laws. Hence, from this perspective tool use is a behavior as all other behaviors and does not require a specific label nor a specific toolbox for its understanding.

Which of these psychological processes are specific to tool use? Which psychological processes involved in tool use are specific to Homo sapiens? From the ecological-dynamical approach it is straightforward to see that processes underlying tool use are not species specific. This is supported by the notion that affordances can be perceived by animals, as is specifically shown by Wagman et al. (Wagman et al., 2018, 2017). Moreover, self-organization is omnipresent as an organizing principle in the animate and the in-animate world. However, this does not mean that the same tooling behavior will be observed among species. That is, Mangalam et al. (2020) showed differences in striking behavior in nut-cracking for bearded capuchin monkeys, expert humans and novice humans. Since these three groups have different organismic constraints, it is to be expected that their effectivities differ, their affordances differ and that the actualization of the affordances by the synergies differ. In sum, two basic notions of the ecological-dynamical approach, affordances and selforganization, play an essential role in tooling and non-tooling behavior among different species. Therefore, there is no principle reason why tool use cannot be understood with similar mechanisms as non-tooling behavior and over different species.

What must be the direction for future work on tool use? The current paper only takes the first steps in applying an ecological-dynamical approach to tool use. This approach needs to be expanded to a broader range of behaviors and tools to be able to give a full-blown account of tool use. Here we present three possible routes in which the approach could be further expanded in the future. First, our earlier studies regarding synergies in rod reaching (Valk et al., 2016; van der Steen and Bongers, 2011) can be taken one step further. A defining characteristic of a synergy is not just co-variation of joint angles, but also the actual location in joint space where the synergy emerges (Tuitert et al., 2020; Wissing et al., 2020). Studying whether, and if so how, the location in joint space where the synergy emerges depends on the tool's properties could further our understanding of the roles of tool constraints in the emergence of synergies.

Second, a topic that is relevant to understand tool use but has not been emphasized in this paper so far is the ability of organisms to construct tools. Tool construction has been studied in the ecological-dynamical framework by Van Dijk \& Bongers (2014), who examined the emergence of an action system when participants constructed new tools from available objects. Since affordances are prospective, they should be particularly apt to explain tool construction. A future line of research could focus on framing the problem of tool construction in terms of perceiving and actualizing affordances to expand the ecological-dynamical framework in the direction of tool construction.

Third, tool use has often been approached as problem solving behavior. Several approaches of tool use therefore incorporated cognitive mechanisms related to reasoning to give a full account of tool use (cf. Osiurak et al., 2010, 2017). Recently attempts have been made to broaden the ecologicaldynamical approach to higher cognitive behaviors, such as action selection (Cox and Smitsman, 2019; Dineva and Schöner, 2018), representations (Golonka and Wilson, 2019) and language (RączaszekLeonardi et al., 2018; van den Herik, 2021). These expansions of an ecological-dynamical approach should in the future make it possible to explain aspects of tool use that are currently explained using cognitive mechanisms. 
Together these developments should enable us to advance a full-blown account of tool use 779 starting from the principles of an ecological-dynamical perspective. We believe that the starting point 780 of such an account should lie in that tools are incorporated in synergies, and as such determine 781 effectivities and affordances. 


\section{More than just tool use}

\section{Dietrich Stout}

\section{Overview}

Humans have been described as tool-making animals but what is really distinctive is the broader realm of human technology. Technology is a broad and variable behavioral domain spanning a spatiotemporal range from neurons to institutions. Understanding its cognitive foundations will require comparative research across diverse technologies unified by an overarching evolutionarydevelopmental theoretical framework. This perspective highlights interactions between technological production, collaboration, and reproduction and the key role played by internal models for action prediction and synchronization.

What constitutes tool use (and is that the right question) ? Many animals make and use tools, but humans are distinctive in the complexity, diversity, sophistication, omnipresence, and obligatory nature of our reliance on tools. Simply put, other animals use tools but only humans have technology. Indeed, humans inhabit a uniquely technological niche that we ourselves have constructed (Stout and Hecht, 2017), and which continues to shape our biology, behavior, and cognition. Attention to this broader technological sphere is clearly warranted. For example, Osiurak and colleagues (Osiurak and Heinke, 2018; Osiurak and Reynaud, 2020a) have argued that the prevailing conception of tool-use as object manipulation is too narrow because it excludes 'tools' such as machines, computers, containers, and structures and fails to emphasize a uniquely human capacity for 'technical reasoning.' Osiurak et al. propose neologisms like intoolligence (Osiurak and Heinke, 2018) to describe this broader sphere of investigation but the existing term technology might prove more apt if its meaning can be suitably constrained.

One approach is to ground the concept in an evolutionary perspective. Humans have evolved a tightly integrated adaptive strategy (Kaplan et al., 2010) in which a focus on high-value, difficult-to-
acquire food resources provides the surplus nutrition needed to fund growth, survival, and reproduction, and is in turn enabled by the increased longevity and brain size that allow teaching, learning, and the cultural evolution of increasingly effective skills, knowledge, and equipment. This human-constructed niche is thereby populated by increasingly complex technological systems focused on material production. This evolutionary perspective converges with the 'technological systems' approach in the social sciences, which identifies a technology as an integrated system of hardware, people, skills, knowledge, social relations, and institutions (Dusek, 2006; Hughes, 1987).

On this view, technology comprises socially reproduced activities involving the manipulation and/or modification of objects to enact changes in the physical environment (Stout, 2013). This extends beyond simple tool use to encompass longer causal chains involving: 1) the coordinated activity of many individuals, 2) use of objects and materials in a wide range of roles other than as hand-held instruments, and 3) processes of social reproduction that sustain and elaborate technological systems. Communication is thus essential to technological systems but is not their ultimate goal. Technologies primarily pursue materially instrumental tasks to achieve physical changes in the world and only incidentally involve communicative tasks that seek to alter thoughts, behaviors, and/or experiences (cf. Legare \& Nielsen, 2015). We could thus speak of technologies for the production of communications tools such as books or musical instruments but not for practices of teaching, storytelling, or musical composition. Systematic approaches to communications might then be termed 'arts' or 'sciences' rather than technologies. This semantic distinction is important because materially instrumental goals are shaped by relatively invariant physical constraints whereas the communicative goals must adapt to human psychology in the context of specific cultural systems of meaning. They will thus tend to implicate different cognitive processes (Finkel et al., 2018; Fischer et 
831 al., 2016; Tylén et al., 2016), learning strategies (Heyes, 2016; Kendal et al., 2018; Legare \& Nielsen, 832 2015), and cultural evolutionary dynamics (Derex and Mesoudi, 2020).

833 What psychological processes are involved in technology? Attempts to specify a critical 'essence' 834 of technology have invoked everything from skilled prehension (Buxbaum, 2017), to causal reasoning 835 (Osiurak and Reynaud, 2020a), mental time travel (Suddendorf et al., 2018), imitation (Derex et al., 836 2019), and mentalizing (Tomasello et al., 1993). A technological niche perspective recognizes all these 837 as relevant and interacting. For example, the sophistication of human technology is often attributed to 838 a process of incremental improvement termed as cumulative cultural evolution (CCE) (Mesoudi and 839 Thornton, 2021). CCE is widely believed to require 'high fidelity' social reproduction dependent on 840 mentalizing and/or imitation to enable the lossless accumulation of innovations (Derex et al., 2019; Tomasello et al., 1993). In the case of technology, however, capacities for causal and analogical reasoning (Gentner and Hoyos, 2017; Osiurak and Reynaud, 2020a), cognitive control (Gönül et al., 2018; McDougle et al., 2016; Stout et al., 2015), memory (Gruber and Ranganath, 2019), and perceptual-motor control (Sánchez et al., 2017) will often be implicated in the generation, identification, and retention of beneficial innovations (Legare and Nielsen, 2015; Miu et al., 2020). Insofar as innovation requires expertise, processes of knowledge reproduction (Gentner and Hoyos, 2017; Pan et al., 2020), skill acquisition (Gowlland, 2019), and innovation (Legare and Nielsen, 2015) will be thoroughly intertwined (Osiurak and Reynaud, 2020a; Stout and Hecht, 2017). Finally, the same capacities for intersubjectivity (Tomasello et al., 1993) and interactive synchrony (Pagnotta et al., 2020; Pan et al., 2020) that support the social reproduction of technology also underpin cooperation and coordination (Hill et al., 2009; Powers et al., 2016) that enable the complexity of collective human technologies to so far exceed that of individual animal tool-use. These complex interactions are organized around three key features of technology: production, collaboration, and reproduction.

Production. The materiality of technological production allows the open-ended accumulation of components and procedures (Derex and Mesoudi, 2020; Stout, 2013) and provides a durable medium for collaboration across time and space. Objects and infrastructure embody information and persist across generations, constituting a novel channel of cultural transmission and evolutionary inheritance (Laland et al., 2015). Technological artifacts and situations also scaffold cognition by externalizing information representation and manipulation and cuing the retrieval of appropriate event schemas (Barbey et al., 2009; Newen et al., 2018; Stout and Hecht, 2017).

Neuroscientific studies have largely focused on simple, hand-held tool use, leading to the identification of a dorsal occipital-parietal-frontal pathway instantiating spatial and kinematic object+body (Martel et al., 2016) models for action planning and perception and a ventral occipitaltemporal pathway representing semantic information about properties and dynamics (Orban and Caruana, 2014; Stout and Hecht, 2017). This has now been extended to a broader account of objectdriven cortex (Yildirim et al., 2019) including object and scene perception in ventral temporal cortex and intuitive physical reasoning about object dynamics (motion, support, collision) in frontoparietal cortex (Fischer et al., 2016). This "physics engine in the brain" is an assembly of regions involved in generating internal models for action planning (McNamee and Wolpert, 2019) and in cognitive control more generally (cf. frontoparietal control network; Dixon et al., 2018; Ptak et al., 2017).

Implicit technical reasoning supported by frontoparietal cortex may be sufficient for everyday tool use (Osiurak and Heinke, 2018), but more complex activities like the tool making and design impose additional demands (Ball and Christensen, 2019; Stout et al., 2015). These include prospection for future planning (Suddendorf et al., 2018), analogy and relational reasoning to find novel solutions (Ball and Christensen, 2019; Vendetti and Bunge, 2014), and metacognitive monitoring of strategic suitability and progress (Ball and Christensen, 2019) all of which involve a shift in attention from external stimuli to internal cognition that is thought to be supported by dynamic interactions between frontoparietal control and the default mode networks (Dixon et al., 2018).

Collaboration. Technology is most clearly distinguished from simple tool-use by its coordination of multiple individuals over extended periods of time and space. Small scale coordination 
882

883

884

885

886

887

888

889

890

891

892

893

894

895

896

897

898

899

900

901

902

903

904

905

906

907

908

909

910

911

912

913

914

915

916

917

918

919

920

921

922

923

924

925

926

927

928

929

930

931

932

between individuals relies on reciprocal prediction achieved by interpersonal coupling of internal forward models for anticipatory motor control (Curioni et al., 2019). This coupling can occur at multiple levels of abstraction (Hasson and Frith, 2016) and provides a key mechanism supporting the implicit mentalizing, empathy, communication, learning, and social affiliation (Alcalá-López et al., 2019; Hasson and Frith, 2016; Pan et al., 2020; Shamay-Tsoory et al., 2019) that in turn support larger scale collaboration. In addition to implicit processes of social alignment (Shamay-Tsoory et al., 2019) collaboration may require explicit negotiation to allocate responsibilities and agree on plans of action (Bang et al., 2017; Mathieu et al., 2017). This may involve metacognitive strategies such as confidence matching, deference to status or experience, and open discussion (Bang et al., 2017; Bang and Frith, 2021; Miu et al., 2020; Shea et al., 2014). Finally, collaboration may be enforced (Bang et al., 2017; Hughes, 1987) by explicit institutional rules and authority, which are in turn the emergent product of interactions between and within smaller groups (Powers et al., 2016; Pryor et al., 2019).

Reproduction. Technological learning is a protracted, collaborative process (Gobet, 2015; Gowlland, 2019; Pargeter et al., 2019; Suddendorf et al., 2016) reflective of demands for precise control of physical contingencies during material production. This problematizes dichotomies of social vs. asocial learning (Galef, 2013; Heyes, 2018), product vs. process copying (e.g., Tennie et al., 2009), and 'blind' vs. guided innovation (Mesoudi, 2021) that have been prevalent in culture evolution research. This is exemplified in technological apprenticeship (Gowlland, 2019; Sterelny, 2012), in which an extended program of alternating social learning and individual practice (Stout, 2013; Whiten, 2015) enables the reproduction increasingly sophisticated skills. Such learning is scaffolded by everything from the exemplar artifacts, available tools, recurring situations, and observable behaviors of culturally constructed 'learning niches' (Flynn et al., 2013; Fragaszy et al., 2013; Stout and Hecht, 2017) to intentional demonstration, explicit instruction, and affective feedback from teachers (Kline, 2015) guides learners to re-create increasingly sophisticated skills though deliberate practice over extended periods, with each round of individual practice allowing deeper appreciation of the available social information.

The resulting expertise combines internal models for efficient action perception, control, and prediction (McNamee and Wolpert, 2019; Sokolov et al., 2017) with flexible task-related, hierarchical knowledge structures of increasing depth and complexity (Gobet, 2015; Stout, 2013). The relevance of each to particular technologies will help determine the efficacy of different learning strategies such as trial-and-error experimentation (Truskanov and Prat, 2018), end-product emulation (Reindl et al., 2017), body movement mimicry (Heyes, 2018; Tennie et al., 2009), intention sharing (Tomasello et al., 1993), and various forms of social scaffolding and teaching (Kline, 2015). Rather than one key reproductive mechanism we should thus expect context-dependent diversity (Caldwell, 2020).

Which of these psychological processes are specific to technology? By the definition proposed here, technology is an evolutionarily relevant domain of human activity rather than a discrete process or capacity. As such, technological cognition is "soft-assembled" from neurocognitive mechanisms and systems as they become behaviorally relevant, rather than hard wired as a dedicated system. Thus, there would be no particular processes specific only to technology although the functional networks recruited by various technologies might be expected show a family resemblance distinct from other behavioral domains. On the other hand, the definition proposed here is motivated by specific evolutionary hypotheses positing that key processes supporting modern technology are elaborated in humans specifically due to selection on technological capacity and aptitude (Stout \& Hecht, 2017). In this evolutionary sense, processes such as intuitive physical reasoning, kinematic monitoring of self and other, and even interactional synchrony would be specifically 'for' technology.

Which psychological processes involved in technology are specific to Homo sapiens? It is not clear that any of the processes involved in supporting technology are qualitatively unique to humans. Perhaps the strongest case could be made for cognitive mechanisms like explicit theory of mind, metacognitive strategies, or analogical reasoning that may themselves be products of cultural evolution in a fully linguistic species (Heyes, 2018). It has, however, been said (although it is unclear by whom) that "Quantity has a quality all its own." Humans may not be qualitatively unique on any one 
933

934

935

936

937

938

939

940

941

942

943

944

945

946

947

948

949

950

951

952

953

954

955

956

957

958

959

960

961

962

963

964

965

966

967

968

969

970

971

972

973

974

975

976

977

978

979 dimension of technology but we are quantitatively exceptional on so many different dimensions at the same time that piecemeal adaptive accounts seem to miss a bigger picture.

As reviewed above, exceptional human capacities for skilled interaction with the physical world appear central to the evolution and development of many key aspects technological cognition. Stout and Hecht (2017) thus suggest a Perceptual Motor Hypothesis (PMH) for the evolutionarydevelopmental-cultural construction of human cognition from ancient primate systems for body awareness and sensorimotor engagement with the world. These systems are early-developing (Baum et al., 2020) and directly engaged with the (internal and external) sensory periphery (Margulies et al., 2016), making them a nexus for interaction between externalizing processes of technological niche construction and internalizing processes of neurocognitive development (Byrge et al., 2014; Flynn et al., 2013; Heyes, 2018; Kennedy et al., 2017). According to the PMH, enhanced human sensorimotor acuity and the experiences this enables guide construction of the internal models and intuitive physics required for technological production. Sensory predictions by these models support the sense of agency and self-other discrimination (Haggard, 2017) that underpin human self-awareness, imitation, social cognition, and empathy (de Guzman et al., 2016) and ultimately the development of "Theory of Mind" capacities (Heyes, 2018), all of which support further technological collaboration, reproduction, and biocultural evolutionary feedback. According to this view, the proper study of technology must cross levels of analysis from neurophysiological dynamics to the cultural evolution of norms and institutions in order to seek unity in diversity by identifying the common processes underlying diverse outcomes across different real-world contexts.

What must be the direction for future work on tool use? Broadly speaking, a future "cognitive science of technology" would be a comparative science seeking to identify patterned relations between contexts, mechanisms, and functions across superficially diverse technologies. This means embracing the real-world complexity, variation, and contextual particularism (Matusz et al., 2019) of technological behaviors. Among the many challenges facing this endeavor are the large spatial and temporal scale of many technological phenomena, which may require long-term study and a combination of experiments (e.g., Pargeter et al., 2019), ethnographic case studies (e.g., Gowlland, 2019), and comparative analyses (e.g., Koster et al., 2020). One more specific direction could be to test the PMH. This would require expanding our surprisingly limited understanding of perceptual-motor variation across primates as well as the proposal that patterns of human technological diversity can be explained in relation to underlying variation in perceptual-motor processes, demands, and developmental interactions. One critical test of the PMH would be the extent to which learning and reasoning about non-mechanical technological properties (e.g. chemical reactivity) rely on concrete perceptual-motor simulation vs. abstract symbolic and analogical thinking (Brand et al., 2021).

Such ambitious aims are increasingly plausible with the development of new methods for the study of behavior and cognition in natural contexts. Techniques such as motion tracking and EEG (Haar et al., 2020; Ladouce et al., 2019) are promising for the study of technological production, while methods for the study of interactional synchrony (Pan et al., 2020; Schirmer et al., 2021) offer insight into mechanisms of small-scale collaboration and social reproduction. At a larger scale, smartphonebased digital phenotyping methods (Onnela, 2021) might be adapted to study individual experience of and engagement with real world technological situations and institutions. Finally, performance in unconstrained real-world situations can be related to individual differences and/or experience-related plasticity in neuroanatomical, cognitive, affective, and perceptual-motor traits assessed in the lab (e.g., Hecht et al., 2015; Prat et al., 2020). This will require novel methods for quantifying technological performance (e.g., Pargeter et al., 2019; Stout et al., 2018) but offers a critical links between structure, function, and behavior needed to place variation in a broader evolutionary and developmental frame (Stout and Hecht, 2017). 
The technical-reasoning hypothesis

\section{François Osiurak}

\section{Overview}

Technical reasoning refers to the ability to reason implicitly about physical object properties (see Osiurak et al., 2010, 2020; Osiurak \& Badets, 2016). This reasoning is both causal (i.e., predicting the effects on the environment) and analogical (i.e., transfer of what is understood from one situation to another). It is based on non-verbal knowledge, called mechanical knowledge, which contains information about physical principles/mechanical actions (e.g., leverage, cutting, percussion). Technical reasoning is much more than spatial reasoning, which can consist, for instance, in determining whether a car can pass between two trees or whether two puzzle pieces can be arranged together. Indeed, it involves the understanding of the material dimension of objects (e.g., hardness, sharpness), which is the basis for the emergence of mechanical actions. Technical reasoning is involved in any manifestation of human materiality such as the use of physical tools (e.g., stone tool, hammer), but also tool making or construction (e.g., building a shelter). It is also thought to be specific to the human lineage. Thus, because it allows humans to discover and master a great amount of technical content, its emergence over evolution might explain why cumulative technological culture, that is the increase in efficiency and complexity of tools and techniques over generations, has been observed only in human populations (Osiurak and Reynaud, 2020b). In the following lines, I present the technical-reasoning hypothesis in more detail ${ }^{1}$.

What constitutes tool use? The technical-reasoning hypothesis does not really confer any special status to tool use because it considers it as one of the manifestations of human materiality in the same ways as tool making or construction (or any technical devices that need the use of natural forces such as wind or fire). In this respect, as argued by Beck (1980) and Shumaker et al. (2011), any definition of tool use necessarily refers to a behavioral description, which does not imply any psychological or biological prerequisites. Thus, it is not because two species exhibit tool behavior that the same cognitive processes are at work in both species (Osiurak and Heinke, 2018). Note that the same is true for tool making or construction behavior. For these reasons, the technical-reasoning hypothesis is in line with the behavioral definitions of tool use, tool making, and construction proposed by Beck (1980) and more recently updated by Shumaker et al. (2011; Table 1). As explained, although the technical-reasoning hypothesis assumes that, in humans, technical reasoning is involved in any manifestation of human materiality (i.e., tool use, tool making, and construction), it nevertheless recognizes that tool use is characterized by an additional mechanism, that is, distalization (Osiurak and Federico, 2020). Distalization refers to the fact that, once a tool is grasped appropriately to be used, the natural effector (usually the hand) is no longer the end-effector. Instead, this end-effector becomes the active part of the tool, that is, the part of the tool that is used to act upon another object (e.g., the head of the hammer). Thus, there is an attentional shift, from the natural effector to the active part of the tool, while it is still the hand that needs to be controlled (for evidence for this distalization mechanism, see Cardinali et al., 2009; Iriki et al., 1996; Maravita et al., 2001; Maravita \& Iriki, 2004; Miller et al., 2018; Osiurak et al., 2017). This distalization mechanism also implies that the user needs to control the degrees of freedom of the body-tool system differently to the body-only system, a

1 I will limit the discussion on tool use to physical tool use (e.g., stone tool, hammer, fork; also called free tool use in Osiurak \& Heinke, 2018). In other words, I will not discuss the use of arbitrary tools (i.e., interface-based technologies; e.g., washing machine, computer, smartphone) or assistive tools (i.e., "autonomous" technologies; e.g., heating system), that is, two phylogenetically recent categories of tools that need technical reasoning to be made but, at best, procedural or associative learning to be used (for more discussion on these tool categories; see (Osiurak, 2020; Osiurak and Heinke, 2018). In this way, they differ from physical tool use, which need technical reasoning to be made and used. The terms tool use and physical tool use will be employed here interchangeably. 
1022 phenomenon called tooling (Fragaszy \& Mangalam, 2018; Mangalam, 2016; Mangalam \& Fragaszy, 1023 2016). Importantly, this distalization mechanism is not unique to humans, which can explain why tool 1024 use can also be reported in nonhuman species. In broad terms, this distalization mechanism is 1025 orthogonal to technical reasoning in that it only allows a species to use tools, but it does not imply that 1026 this species possesses technical-reasoning skills.

1027 What psychological processes are involved in tool use? Four cognitive functions are mainly 1028 involved in human tool use, each dedicated to a specific role (Fig. 5): Technical reasoning, motor 1029 control, semantic memory, and planning ${ }^{2}$. The role of technical reasoning is to generate technical 1030 solutions to solve physical problems, which can be novel (e.g., to get a small ball that rolled under a 1031 couch) or familiar (e.g., to peel an apple). In this respect, the technical-reasoning hypothesis diverges 1032 from related proposals, which consider that technical-reasoning-like processes are primarily involved 1033 in novel situations and, at best, can be employed as an alternative strategy in familiar activities (e.g., 1034 Buxbaum, 2017; Caruana \& Cuccio, 2017; Martin et al., 2016; Norman, 2002). According to the 1035 technical-reasoning hypothesis, technical-reasoning skills support both familiar and novel tool use, 1036 even if the process can be faster for familiar activities than for novel situations. Technical reasoning is 1037 1038 1039 1040 1041 1042 1043 1044 1045 1046 1047 1048 1049 1050 1051 1052 1053 1054 1055 1056 1057 1058 critical to determine the appropriate mechanical actions as well as to select the appropriate tools and objects to solve these problems. The outcome of technical reasoning is a mental simulation of the mechanical action to be performed (e.g., the motion of a knife on an apple). However, simulating a mechanical action is not sufficient to realize it in the physical environment. This realization needs the selection and on-line control of the most appropriate motor actions. This is the role of the motor-control system, which is unaware of the goal of the action (e.g., tool use, object transport). If someone intends to perform back-and-forth movements with a knife on an apple, this is the expected effect, which constrains the motor actions selected within the motor-control system. Likewise, if someone has the idea to move an object from one location to another, the expected effect is the motion of the object, which also constrains the motor actions selected. Said differently, the motor-control system is only concerned with the economy of motor actions performed. Because tools and objects are not always directly available, the user needs to get them from other places. The role of semantic memory is to organize the search in episodic memory to know and, thus, to remember where to get the tools and objects appropriate for the ongoing activity (for discussion, see Osiurak, 2014, 2017). Indeed, knowledge about semantic categories (e.g., cooking, washing) is helpful to carry out an efficient search in episodic memory and to think of about tools and objects that are not here now (see Tulving, 1985). Finally, tool-use activities usually require a sequence of mechanical actions that can involve several tools and objects. An individual can generate these different mechanical actions in an unordered way. The role of planning skills is to rearrange this sequence in an ordered way so that to optimize time and to avoid potential fatal errors (i.e., the realization of a mechanical action that blocks the realization of another one). Thus, planning skills allow the individual to foresee future changes of the environment.

Evidence for the technical-reasoning hypothesis has come from the study of left braindamaged patients, which has revealed a strong relationship between the ability to use and select familiar tools as well as to use, select and make novel tools to solve mechanical problems (Goldenberg \& Hagmann, 1998; Goldenberg \& Spatt, 2009; Osiurak et al., 2009; for a review, see Osiurak et al., 2020). This behavioral association has also been confirmed by brain lesion studies, which have indicated that difficulties in using both familiar and novel tools are associated with damage to the brain area PF within the left inferior parietal lobe (Goldenberg and Spatt, 2009; Martin et al., 2016a; Mengotti et al., 2013; Salazar-López et al., 2016). Two recent neuroimaging meta-analyses have also revealed that the left area PF is preferentially and selectively activated when healthy participants focus on the mechanical actions between a tool and an object as well as when they observe others using tools (Reynaud et al., 2019, 2016). In other words, technical-reasoning skills could mainly be supported by the left area PF, allowing humans to generate and understand mechanical actions. By contrast, the ability to select the appropriate motor actions (to use tools or not)

2 I am aware that motor control is not a cognitive function, strictly speaking. Nevertheless, for the sake of simplicity, I will consider it as one of the four main "cognitive" functions discussed in this section. 
1072

1073

1074

1075

1076

1077

1078

1079

1080

1081

1082

1083

1084

1085

1086

1087

1088

1089

1090

1091

1092

1093

1094

1095

1096

1097

1098

1099

1100

1101

1102

1103

1104

1105

1106

1107

1108

1109

1110

1111

1112

1113

1114

1115

1116

has been associated with more superior parietal structures and particularly the intraparietal sulci (e.g., Reynaud et al., 2016; Vingerhoets, 2014). Concerning semantic memory, a significant body of evidence has shown that semantic memory is neither necessary nor sufficient to actually use familiar or novel tools with objects (e.g., Buxbaum, 2017; Hodges et al., 2000; Lesourd et al., 2016; Silveri \& Ciccarelli, 2009; for discussion, see Osiurak \& Badets, 2016). In broad terms, this indicates that semantic memory is not useful to determine appropriate mechanical actions. However, the loss of semantic memory after temporal lobe lesions can impair the ability to use tools presented in isolation (Baumard et al., 2016; Hodges et al., 2000; Osiurak et al., 2008; Sirigu et al., 1991). thereby suggesting that semantic memory is first and foremost involved in the ability to link a tool with a specific usage (Osiurak, 2014). Finally, it has been found that patients with frontal lobe lesions/dysexecutive syndrome are impaired when they have to perform complex tool-use activities that require a sequence of mechanical actions and not when they have to solve less complex tool-use activities that require only one mechanical action (e.g., Goldenberg et al., 2007). To sum up, the four main cognitive functions that support human tool use are each dedicated to a specific role and associated with a specific neural basis (Technical reasoning: To solve physical problems/Left area PF; Motor control: To perform appropriate motor actions/Intraparietal sulcus; Semantic memory: To get absent tools and objects/Temporal lobes; Planning: To rearrange sequences of actions in an economical way/Prefrontal cortex).

Which of these psychological processes are specific to tool use? None of the four aforementioned cognitive functions are considered as specific to tool use, except the distalization mechanism of the motor-control system, which could constitute the very essence of tool use (see above). Indeed, as explained, technical reasoning is also at work when humans make tools or build constructions. The motor-control system is unaware of the goal of the action. Semantic memory is involved mo11re generally in our knowledge about the world and is not critical to actually use tools with objects. Finally, planning skills are in charge of optimizing time when a sequence of actions is performed, irrespective of whether this sequence is tool-centered or not (e.g., construction behavior, but also when people play chess or optimize their shopping trips).

Which psychological processes involved in tool use are specific to Homo sapiens? There is a tendency in the animal cognition literature to give to nonhuman tool-using species a special status, as if tool use reflected a specific sign of intelligence. As argued by Hansell and Ruxton (Hansell and Ruxton, 2008), this status might be exaggerated because based on a confusion or even a kind of bijection between tool use and specific physical-cognitive skills. As explained above, tool use could be observed in any species that possesses a distalization ability. However, this ability is orthogonal to the level of physical understanding that the species has. In this way, the technical-reasoning hypothesis obviously recognizes that tool use is not specific to humans. However, it also stresses that the study of tool use is not sufficient to understand how humans and nonhuman animals are able to understand their physical environment. As a matter of fact, the technical-reasoning hypothesis suggests that even if some animals could possess some of the components of technical reasoning (e.g., the causal component), technical reasoning in its entirety could be specific to humans. This claim is based on evidence from micro-society paradigms ${ }^{3}$, which have shown that cumulative performance can emerge over generations even when individuals only transmit the product made (e.g., a tower or a basket; i.e., reverse engineering; (Caldwell and Millen, 2009; Derex et al., 2019; Osiurak et al., 2021b; Zwirner and Thornton, 2015). Technical-reasoning skills (assessed with psychotechnical tests) have also been found to be the best predictor of cumulative performance in these micro-society experiments (De Oliveira et al., 2019; Osiurak et al., 2021a, 2020a, 2016). Conversely, cumulative performance

3 Micro-society paradigms aim to investigate cultural evolution in laboratory (e.g., Caldwell \& Millen, 2009; Morgan et al., 2015). The most popular version is the transmission chain paradigm. The task can be to optimize the speed of a wheel that descends an inclined track by moving the four weights placed along each spoke of the wheel (Derex et al., 2019; Osiurak et al., 2021b). The first participant of the chain performs the task. The second participant can scrutinize the product of (i.e., reverse-engineering), observe the action made by, or communicate with the first participant before performing the task themselves. Then, the third participant does the same with the second one, and so on. 
1117 observed in this kind of paradigm is also accompanied by an increase in understanding of the physical 1118 system (Osiurak et al., 2021b). Taken together, these results indicate that technical-reasoning skills 1119 could have played a key role in cumulative technological culture, namely, a phenomenon that is considered as unique to the human lineage ${ }^{4}$. In sum, although tool use is not unique to humans, it might be nevertheless be specific in humans because of its 'recycling' by technical-reasoning skills.

1122 What must be the direction for future work on tool use? There is a crucial need in the literature to orthogonality at a cognitive level. It is one thing to be able to manipulate a tool to use it with an object and another to understand the physical effects of this interaction. In this respect, three distinct lines of research should be developed. The first line should consist in extending the question of physical understanding in human tool use to tool making and construction behavior. One of the goals of this extension can be to test whether technical-reasoning skills and its neural correlates, that is the left area PF, is really involved in any manifestation of human materiality. More generally, this could allow emergence of a specific field in cognitive sciences investigating cognitive skills needed to modify the physical environment, that is, technition (Osiurak et al., 2020b). The second line should consist in deepening our understanding of the cognitive mechanisms that are required to use tools (potentially the distalization mechanism). Taken together, these two lines of research can help us to specify the cognitive components associated with tool-use skills versus technical-reasoning skills. Finally, a third line of research could explore whether a certain link can exist between both. For instance, even if these two kinds of skills are orthogonal, it remains very plausible that the ability to manipulate tools can favor the exploration of the physical environment and can be an important prerequisite for the emergence of technical-reasoning skills in a given species. Thus, these three lines of research should be particularly useful to understand the specific trajectory of technological evolution in the human lineage on the basis of a plurality of methods and techniques from distinct but complementary disciplines, such as neuropsychology, cognitive neuroscience, developmental and comparative psychology, archaeological and anthropological sciences.

4 Studies on New Caledonian crows indicate that some signs of cumulative technological culture have been observed in this species, which is for its elaborate causal-reasoning skills (Rutz and Hunt, 2020; Taylor and Jelbert, 2020). In other words, although the technical-reasoning hypothesis in its strong form posits that both cumulative technological culture and technical-reasoning skills might be unique to humans, much more evidence is needed to demonstrate it (or to invalidate this claim). 


\section{Discussion}

1144 Multiple lines of scientific and philosophical investigation are concerned with the development and expression of humans' engagement with tools and its relation to other dimensions of human experience. At first sight, these multiple lines of inquiry (including those described by the six author groups in this collection) may appear to be incongruent. However, as we shall discuss below, these seemingly divergent perspectives converge on some common themes and consequently foster a more comprehensive, interdisciplinary understanding of tool use than any of these perspectives would (or could) on their own. It is to these seeming consistencies and their resolution that we turn now.

What constitutes tool use? The six author groups addressed this fundamental question differently. Mangalam and Fragaszy provide a definition in which tooling is a deliberate act in which the bodyobject system produces a mechanical effect on a target. This definition necessitates that the actor, tool, and target physically contact each other during the activity of tooling and that the act of tooling lasts only as long as this spatial relationship is maintained (Fragaszy \& Mangalam, 2018; Mangalam \& Fragaszy, 2016). The authors justify this narrow definition because it affords analytical clarity.

Wagman and Day expand the scope of this definition by including any interaction with an intermediary object that changes the ability to perceive and actualize affordances in the context of performing a goal-directed behavior. Consequently, Wagman and Day classify behaviors as tool use that Mangalam and Fragaszy would not,such as throwing objects at a target and using a wheelbarrow to transport material. Except for this discrepancy, Wagman and Day's views are mostly compatible with those of Mangalam and Fragaszy_both rooted in the Gibsonian concepts of affordances and information detection. Bongers' perspective is rooted in these concepts as well: tool use occurs when an environmental object temporarily alters the capacity for action, requiring the new coordination patterns in the act of performing that action (cf. Profeta \& Turvey, 2018).

Kelty-Stephen goes beyond these authors by proposing that tool use is an intermittent cascade of contingencies across a permeable animal-environment boundary. This permeability allows tool movement to inherit the multifractal fluctuations inherent in limb movement and return new multifractal fluctuations to those limbs (Mangalam, Carver, et al., 2020a, 2020b). Fluctuations flow through a continuous medium spanning the organism, tool, and environment via informational contact (acoustic, mechanical, optical). So, for Kelty-Stephen (like Wagman \& Day), tool use does not necessitate continual mechanical contact between the object and the body.

Stout and Osiurak, respectively, take an entirely different tack in defining tool use. Stout finds no utility in defining tool use per se and instead chooses to subsume tool use under the broader umbrella of technology. For him, technology (but not tool use) is a uniquely human endeavor-tool use (considered on its own) fails to capture the uniquely human capacity for 'technical reasoning.' Stout's view is consistent with that of Osiurak for whom 'intelligence' is the intelligent use of technological innovations including, but not limited to, tools (Osiurak and Heinke, 2018; Osiurak and Reynaud, 2020a).

Osiurak does not confer any special status to tool use because it is merely one of the ways in which technical reasoning is brought to bear in human materiality. However, Osiurak suggests that tool use requires an additional mechanism beyond technical reasoning: distalization (Osiurak and Federico, 2020). Distalization is a shift in the locus of perception-action from the body to the active part of the tool, and several author groups highlighted the centrality of this phenomenon in using tools. A key implication is that controlling the body-tool system requires establishing different synergies across different sets of degrees of freedom than controlling the body itself. This aspect of Osiurak's analysis aligns with Mangalam and Fragaszy's embodied theory of tooling (Fragaszy \& Mangalam, 2018; Mangalam \& Fragaszy, 2016), Wagman and Day's description of tools use as the exploitation of an altered animal-environment fit, Bongers' description of tool use as the control of synergies emerging across tool user and tool use task, and Kelty-Stephen's description of the nonlinear interactions across the porous boundary between user and tool. 
Hence, the six author groups differ in how inclusive or exclusive their definition of tool use is. It is important to remember that definitions provide a way of formulating a problem and an approach to solving it. Each of these definitions will yield distinct (though perhaps not entirely incompatible) answers to the following fundamental questions: What psychological processes are involved in tool use? Which of these psychological processes are specific to tool use? Which psychological processes involved in tool use are specific to Homo sapiens?

What psychological processes are involved in tool use? A fundamental question in the study of tool use concerns the psychological processes that underlie this phenomenon, but the divergence among researchers about what constitutes tool use may hamper a coherent answer to this question. Some researchers have investigated the neurophysiological underpinnings of tool use by studying brain-damaged patients (Goldenberg and Spatt, 2009; Lewis, 2006; Wheaton and Hallett, 2007) or noninvasive functional neuroimaging in everyday settings (Bril et al., 2005; Krakauer et al., 2017). Others have done so by conducting comparative analyses of tool use across species (Bril et al., 2012; Mangalam et al., 2020; Mangalam \& Fragaszy, 2016). Unfortunately, any observed pattern of brain activation is mute on the psychological processes reflected by that activation pattern. A lack of a coherent theoretical framework makes it challenging to conduct studies across species that yield testable predictions about the psychological processes involved in tool use. So, we take this opportunity to suggest some degree of convergence on the psychological processes involved in tool use.

For Mangalam and Fragaszy, the psychological processes of interest in tool use are at the level of perception and action-in particular, the functional task demands establishing and maintaining a mechanical interface. In their embodied theory of tooling (Fragaszy and Mangalam, 2018), tool use depends on the perceptuomotor processes related to perceiving spatial relations among objects and surfaces, developing agency over objects attached to the body (the distalization of the end effector), and controlling the bodily degrees of freedom to meet functional task demands. Mangalam and Fragaszy emphasize that these processes occur in the body-object-task-environment system. Moreover, together, these processes distinguish tool use from other instrumental problem-solving.

Similarly, for Wagman and Day, the psychological processes of interest in tool use are at the level of perception and action. They focus their analysis on detecting and exploiting lawfully structured stimulation patterns ('information'). When the animal-environment fit is altered by a tool, the structure encountered at the point of observation provides information about affordances for tool use-whether, when, and how the tool can be used to achieve a goal. The lawfulness of this process entails that information about tool use is invariant over transformations (Hajnal et al., 2007; Mangalam et al., 2017; Wagman et al., 2017; Wagman \& Hajnal, 2014b, 2014a, 2016). Perceiving (and actualizing) such affordances requires spontaneously and temporarily assembling task-specific detection units (and task-specific control units) across body and tool capable of detecting (and exploiting) such information.

Kelty-Stephen takes the lawful grounding of tool use described by Wagman \& Day one step further. The processes that underlie tool use are cascade dynamics-the same generic processes that underlie the behavior of all systems that branch, avalanche, diffuse, or spread apart across multiple temporal or spatial scales. Cascade dynamics are exhibited at all levels of the organization and all levels of scale both within an organism and across the animal-environment system (Goldberger et al., 1990)(Fernandez-Gonzalez \& Zallen, 2011)(Fratzl, 2008). Moreover, the spreading of these interactions across levels and scales of the analysis entails a multifractal structure (e.g., Ingber, 2006) (Turvey and Fonseca, 2014). The waxing and waning of multifractal fluctuations predict cascadedriven coordination of events during tool use (Mangalam, Carver, et al., 2020a, 2020b).

Bongers' analysis is also at the level of perception and action and has the same lawful grounding in specificity relations as Wagman and Day and Kelty-Stephen, respectively. Bongers' focus is on the control of coordinated action in actualizing affordances for tool use. Actualizing affordances (with or without a tool) requires establishing synergies-emergent coordinative patterns among numerous degrees of freedom spanning the task, organism, and environmental constraints. The 
synergies that can emerge under these circumstances determine an animal's capability for acting-its effectivities. An object attached to the body that functions as a tool affects the emergence of synergies, and hence, the effectivities of the action system. Therefore, tool use requires integrating the tool into the synergy emerging in the action system consisting of body-tool. This thesis is consistent with the analysis provided by Mangalam and Fragaszy.

Stout's emphasis on technology rather than tool use per se leads him to consider a broader range of psychological processes that are more intimately tied to traditional cognitive psychology than any of the preceding authors, especially so because of the relationship between technology and culture. At the technological level, these processes include causal and analogical reasoning, cognitive control, memory, and perceptual-motor control in behavioral exploration. At the level of a technological society, these processes are organized around production, collaboration, and reproduction.

Finally, Osiurak posits that tool use is supported by four cognitive functions: technical reasoning, motor control, semantic memory, and planning. Importantly, technical reasoning results in a simulation of the mechanical action performed but not the performance of those actions. Motor control is thus required to select and control the simulated actions. Semantic memory is required to think about tools appropriate for performing the simulated action and where such tools might be located. Planning is required to prospectively organize the actions involving tools in a way that is efficient and as error-free as possible.

So, what are the points of convergence across researchers in their ideas about the psychological processes involved in tool use? All author groups note that tool use is associated with transforming the body into the body-tool system (i.e., all incorporate the notion of distalization of the end-effector). Mangalam and Fragaszy, Wagman and Day, and Bongers each focus on tool use to detect and exploit lawfully structured patterns of energy that provide 'information about' affordances of tools. Kelty-Stephen also grounds his analysis in lawful relations, but shifts focus away from psychological processes per se and toward generic processes of cascade dynamics. Stout and Osiurak, respectively, focus more on processing and cultural transmission of information. Specifically, they identify technical reasoning, semantic memory, and planning as cognitive abilities fundamental to the production, collaboration in, and reproduction of novel tool-use behaviors.

Which of these psychological processes are specific to tool use? This question is important because if any psychological process is specific to tool use, it will imply that a particular developmental and/or evolutionary trajectory enables or facilitates tool use. Indeed, this trajectory is what Mangalam and Fragaszy's definition attempts to uncover. Their definition of tool use attempts to provide a theoretical foundation for making testable predictions about which individuals (and species) can use tools, to what extent they can do so, and under which conditions. For Mangalam and Fragaszy, the three processes that support tool use are: (1) establishing and managing spatial relation(s) between an object attached to the body and a target object; (2) distalization of the end-effector; (3) coordinating the body-object system. They propose that while none of these perceptuomotor processes are specific to tool use, the concurrent involvement all three is a distinctive feature of tool use.

Osiurak posits that four cognitive functions that support tool use (technical reasoning, motor control, semantic memory, and planning), but only one of them-distalization-is specific to tool use. For Wagman \& Day, the lawfully structured patterned energy arrays that provide information about whether, when, and how to move to achieve a goal are invariant across transformations-including whether (or not) a tool is used in the act (e.g., invariant mechanical patterns are informative about controlling limb movement regardless of whether a person is using a hand-held object). Along similar (but perhaps not identical) lines, Bongers and Kelty-Stephen advocate that the processes underlying self-organization in complex tool-using systems are entirely generic, underlying self-organization in all complex systems - human and animal, living and non-living.

For the other authors, however, there are no psychological processes that are specific to tool use. For Wagman \& Day, the lawfully structured patterned energy arrays that provide information about whether, when, and how to move to achieve a goal are invariant across transformations- 
1293

1294

1295

1296

1297

1298

1299

1300

1301

1302

1303

1304

1305

1306

1307

1308

1309

1310

1311

1312

1313

1314

1315

1316

1317

1318

1319

1320

1321

1322

1323

1324

1325

1326

1327

1328

1329

1330

1331

1332

1333

1334

1335

1336 including whether (or not) a tool is used in the act of doing do (e.g., invariant mechanical patterns are informative about controlling limb movement regardless of whether a person is using a hand-held object). Along similar (but perhaps not identical lines), Bongers and Kelty-Stephen advocate that the processes underlying self-organization in complex tool-using systems are entirely generic, underlying self-organization in all complex systems—human and animal, living and non-living.

The distalization of the end-effector when using tools has received attention in various forms, such as the tooltip becoming the end-effector (Arbib et al., 2009; Takahashi and Watt, 2017; Umiltà et al., 2008), changes in the perception of space (Berti and Frassinetti, 2000; Canzoneri et al., 2013), and the extension of sensorimotor processing (Miller et al., 2018). Mangalam and Fragaszy (and to some extent Osiurak) argue that this condition is necessary but not sufficient for tool use. Yet Wagman and Day, Kelty-Stephen, and Bongers' argue that distalization (or any other psychological process) is neither sufficient nor necessary for tool use. How might this be resolved? For Mangalam and Fragaszy, the distalization of the end-effector is a behavioral description associated with the shift of the locus of perceptual and action control from the biological effector (e.g., hand, beak). Hence, any investigation must, at least, explain such patterns of variation in the perceptual and action outcomes of using a tool. For Wagman and Day, Bongers, and Kelty-Stephen, these processes are the same ones that enable perception and movement of the body without a tool (Harrison et al., 2011; Mangalam, Pacheco, et al., 2019; Pagano et al., 1993; Wagman et al., 2017). These two approaches address two distinct sets of questions - one associated with perception-action shift (Mangalam \& Fragaszy, 2016), and the other associated with informational support for perception (Thomas et al., 2019).

Which psychological processes involved in tool use are specific to Homo sapiens? Any special association of tool use with humans depends on whether any psychological process involved in tool use is specific to humans. Otherwise, shared processes would suggest that human tool use differs from that observed in nonhuman species in the degree but not the type of psychological processes.

Mangalam and Fragaszy address tool use at the behavioral level, emphasizing perceptuomotor processes of perceiving spatial relations among objects and surfaces, developing agency over objects attached to the body (the distalization of the end effector), and controlling the bodily degrees of freedom to meet functional task demands. Importantly, Mangalam and Fragaszy suggest that none of these processes are unique to humans-nonhuman species show behaviors supported by each of these processes in isolation. However, they propose that what is unique to humans is the degree to which these processes can be regulated simultaneously and the number of functional parameters that can be controlled in a given tool use task, such as when manufacturing sharp flakes from stone cores (Bril et al., 2015, 2012).

Wagman and Day argue that lawful structuring of energy distributions by the animalenvironment relationship entails that the information about a given affordance (for tool use) is invariant over the sensory apparatus, nervous system, and brain of the animal doing the perceiving. Therefore, any animal capable of detecting (and exploiting) such structure is capable of tool use. For them, any differences in the ability to use tools between humans and non-humans is due to the sophistication of the ecological niche occupied by that species (Gibson, 1979; Turvey, 2018). The more sophisticated the environment occupied by a given species, the more sophisticated the affordances available to that species. This argument explains why nonhuman primates that do not use tools in their natural habitat use tools more frequently when in captivity (Haslam, 2013). Hence, Wagman and Day's thesis is consistent with that of Stout who argues that humans inhabit a uniquely technological environment and (like all animal species) participate in the construction of that environment.

Bongers offers a similar view, arguing that the lawfulness underlying (the self-organization of) perception-action entails that the processes underlying tool use are not (and cannot be) speciesspecific. Any differences between species in their capacity to use tools will be due (in part) to differences in emergent synergies given task, organism, and environmental constraints. Kelty-Stephen also relies on the lawful dynamics of self-organization by drawing an analogy between how an amoeboid slime mold fashions a tool from its bodily degrees of freedom and how a human fashions a tool from some external object. 

qualitatively unique to humans.

Osiurak argues that whereas tool use itself is not a uniquely human phenomenon, some of the psychological processes underlying it (technical reasoning skills) may be so. He argues that these technical reasoning skills have contributed to the cumulative technical culture that is specific to humans.

In summary, although the authors differ significantly in their views on whether any psychological process is specific to tool use, none identified even a single psychological process involved in tool use that is specific to humans. The emerging theme is that the psychological processes that underlie humans' unprecedented capability to use tools, make new tools, and recycle old tools for new purposes are shared in some manner with other species.

What must be the direction for future work on tool use? As noted above, how each author group defines tool use drives how that author group investigates tool use and hence envisions the direction for future work on tool use. Mangalam and Fragaszy suggest that future work must be concerned with the three-component perceptuomotor processes involved in tool use: (1) establishing and managing spatial relation(s) between an object attached to the body and a target object; (2) distalization of the end-effector; and (3) coordinating the body-object system during tool use. Kelty-Stephen suggests applying his perspectives on tool use to a broader set of questions related to whether and how the multifractal geometry of tool use can formalize agency as a cascade-driven process. Examples of such studies include the role of fluctuations within the body (Mangalam, Conners, \& Singh, 2019; Mangalam, Conners, Kelty-Stephen, et al., 2019), the role of fluctuations at the postural center of pressure (Mangalam, Chen, et al., 2020; Mangalam \& Kelty-Stephen, 2020), and the flow of multifractal fluctuations to-and-fro between the body and the tool (Mangalam, Carver, et al., 2020a, 2020b). Bongers makes three specific suggestions about applying the ecological-dynamics approach to the emergence of synergies in tool use, tool construction, and higher cognitive behaviors such as action selection and language. Osiurak and Stout, respectively, also suggest that future work must focus on the processes underlying tool (and technology) use; both suggest taking advantage of noninvasive brain imaging techniques to investigate the neurophysiological underpinnings of tool use. Specifically, Stout highlights that using multiple techniques simultaneously such as motion tracking and electroencephalography (EEG) (Haar et al., 2020) has enormous potential for the study of technological production.

Like Bongers, Osiurak suggests that future work must investigate tool making and tool construction, but for him, the goal of such investigations should be to uncover the technical reasoning skills as well as the cognitive mechanisms that underlie distalization of the end-effector. Wagman and Day propose that future work on tool use focus on the technological, communication, and representation systems characteristic of (tool use in) the human niche. They propose the development of interfaces for teleoperation of devices such as laparoscopic surgical tools, surveillance drones, or phone apps that preserve, enhance, or generate lawful structuring of energy patterns at any point of observation, and how to best train users of such devices (cf. Pagano \& Day, 2020). Similarly, Stout promotes 'real-world technological situations' to investigate relations among structure, function, and behavior. However, he focuses less on the development of technology itself and more on understanding relations between performance in tool-use tasks and individual differences and/or experience-related plasticity in neuroanatomical, cognitive, affective, and perceptuomotor traits assessed in the lab.

In summary, the six author groups with extensive research experience (> 100 years in total) present quite distinct perspectives on the direction of future work on tool use. To a student just beginning his/her research career, or to a seasoned researcher, tool use might not appear as multifaceted a phenomenon with a wide scope for study as this review suggests. All eight authors hope that this review inspires both new and seasoned researchers alike to look again at tool use-a behavior that sparks the feeling of wow in a child successfully hammering a nail for the first time, and that is arguably a keystone of human existence. 


\section{References}

Alcalá-López, D., Vogeley, K., Binkofski, F., Bzdok, D., 2019. Building blocks of social cognition: Mirror, mentalize, share? Cortex 118, 4-18. https://doi.org/10.1016/j.cortex.2018.05.006

Altenhoff, B.M., Pagano, C.C., Kil, I., Burg, T.C., 2017. Learning to perceive haptic distance-to-break in the presence of friction. J. Exp. Psychol. Hum. Percept. Perform. 43, 231-244. https://doi.org/10.1037/xhp0000298

Arbib, M.A., 2012. Tool use and constructions. Behav. Brain Sci. 35, 218-219. https://doi.org/10.1017/S0140525X11002123

Arbib, M.A., Bonaiuto, J.B., Jacobs, S., Frey, S.H., 2009. Tool use and the distalization of the endeffector. Psychol. Res. 73, 441-462. https://doi.org/10.1007/s00426-009-0242-2

Bagorda, A., Mihaylov, V.A., Parent, C.A., 2006. Chemotaxis: Moving forward and holding on to the past. Thromb. Haemost. 95, 12-21. https://doi.org/10.1160/TH05-07-0483

Ball, L.J., Christensen, B.T., 2019. Advancing an understanding of design cognition and design metacognition: Progress and prospects. Des. Stud. 65, 35-59. https://doi.org/10.1016/j.destud.2019.10.003

Bang, D., Aitchison, L., Moran, R., Herce Castanon, S., Rafiee, B., Mahmoodi, A., Lau, J.Y.F., Latham, P.E., Bahrami, B., Summerfield, C., 2017. Confidence matching in group decision-making. Nat. Hum. Behav. 1, 117. https://doi.org/10.1038/s41562-017-0117

Bang, D., Frith, C.D., 2021. Making better decisions in groups. R. Soc. Open Sci. 4, 170193. https://doi.org/10.1098/rsos.170193

Barbey, A.K., Krueger, F., Grafman, J., 2009. Structured event complexes in the medial prefrontal cortex support counterfactual representations for future planning. Philos. Trans. R. Soc. B Biol. Sci. 364, 1291-1300. https://doi.org/10.1098/rstb.2008.0315

Bauer, H., 2001. Use of tools by lions in Waza National Park, Cameroon. Afr. J. Ecol. 39, 317. https://doi.org/10.1046/j.1365-2028.2001.00311.x

Baum, G.L., Cui, Z., Roalf, D.R., Ciric, R., Betzel, R.F., Larsen, B., Cieslak, M., Cook, P.A., Xia, C.H., Moore, T.M., Ruparel, K., Oathes, D.J., Alexander-Bloch, A.F., Shinohara, R.T., Raznahan, A., Gur, R.E., Gur, R.C., Bassett, D.S., Satterthwaite, T.D., 2020. Development of structure-function coupling in human brain networks during youth. Proc. Natl. Acad. Sci. 117, 771-778. https://doi.org/10.1073/pnas.1912034117

Baumard, J., Lesourd, M., Jarry, C., Merck, C., Etcharry-Bouyx, F., Chauviré, V., Belliard, S., Moreaud, O., Croisile, B., Osiurak, F., Le Gall, D., 2016. Tool use disorders in neurodegenerative diseases: Roles of semantic memory and technical reasoning. Cortex 82, 119-132. https://doi.org/10.1016/j.cortex.2016.06.007

Beck, B.B., 1980. Animal Tool Behavior. Garland STPM Press, New York, NY.

Bernstein, N.A., 1967. The Co-ordination and Regulation of Movements. Pergamon Press, New York, NY. 
1432

Bernstein, N.A., Latash, M.L., Turvey, M.T., 1996. Dexterity and Its Development. Lawrence Erlbaum, Marwah, NJ.

Berti, A., Frassinetti, F., 2000. When far becomes near: Remapping of space by tool use. J. Cogn. Neurosci. 12, 415-420. https://doi.org/10.1162/089892900562237

Bird, C.D., Emery, N.J., 2017. Rooks use stones to raise the water level to reach a floating worm. Curr. Biol. 19, 1410-1414. https://doi.org/10.1016/j.cub.2009.07.033

Biryukova, E., Bril, B., 2012. Biomechanical analysis of tool use: A return to Bernstein's tradition. Z. Psychol. 220, 53-54. https://doi.org/10.1027/2151-2604/a000092

Bongers, R.M., 2001. An Action Perspective on Tool Use and Its Development. Printpartners Ipskamp BV, Enschede, Netherlands.

Bongers, R.M., Michaels, C.F., Smitsman, A.W., 2004. Variations of tool and task characteristics reveal that tool-use postures are anticipated. J. Mot. Behav. 36, 305-315. https://doi.org/10.3200/JMBR.36.3.305-315

Bongers, R.M., Smitsman, A.W., Michaels, C.F., 2003. Geometries and dynamics of a rod determine how it is used for reaching. J. Mot. Behav. 35, 4-22. https://doi.org/10.1080/00222890309602117

Borsari, A., Ottoni, E.B., 2005. Preliminary observations of tool use in captive hyacinth macaws (Anodorhynchus hyacinthinus). Anim. Cogn. 8, 48-52. https://doi.org/10.1007/s10071-004-0221-3

Boyd, R., Richerson, P.J., 1996. Why culture is common, but cultural evolution is rare, in: Evolution of Social Behaviour Patterns in Primates and Man, Proceedings of The British Academy, Vol. 88. Oxford University Press, New York, NY, US, pp. 77-93.

Brand, C.O., Mesoudi, A., Smaldino, P.E., 2021. Analogy as a catalyst for cumulative cultural evolution. Trends Cogn. Sci. 25, 450-461. https://doi.org/10.1016/j.tics.2021.03.002

Bril, B., Dietrich, G., Foucart, J., Fuwa, K., Hirata, S., 2009. Tool use as a way to assess cognition: How do captive chimpanzees handle the weight of the hammer when cracking a nut? Anim. Cogn. 12, 217-235. https://doi.org/10.1007/s10071-008-0184-x

Bril, B., Parry, R., Dietrich, G., 2015. How similar are nut-cracking and stone-flaking? A functional approach to percussive technology. Philos. Trans. R. Soc. Lond. B. Biol. Sci. 370, 20140355. https://doi.org/10.1098/rstb.2014.0355

Bril, B., Roux, V., Dietrich, G., 2005. Stone knapping: Khambat (India), a unique opportunity?, in: Roux, V., Bril, B. (Eds.), Stone Knapping: The Necessary Conditions for a Uniquely Hominin Behaviour. McDonald Institute for Archaeological Research, Cambridge, UK, pp. 53-71.

Bril, B., Smaers, J., Steele, J., Rein, R., Nonaka, T., Dietrich, G., Biryukova, E., Hirata, S., Roux, V., 2012. Functional mastery of percussive technology in nut-cracking and stone-flaking actions: Experimental comparison and implications for the evolution of the human brain. Philos. Trans. R. Soc. B Biol. Sci. 367, 59-74. https://doi.org/10.1098/rstb.2011.0147

Buxbaum, L.J., 2017. Learning, remembering, and predicting how to use tools: Distributed neurocognitive mechanisms: Comment on Osiurak and Badets (2016). Psychol. Rev. 124, 346360. https://doi.org/10.1037/rev0000051 
Buxbaum, L.J., 2001. Ideomotor apraxia: A call to action. Neurocase 7, 445-458. https://doi.org/10.1093/neucas/7.6.445

Buxbaum, L.J., Johnson-Frey, S.H., Bartlett-Williams, M., 2005. Deficient internal models for planning hand-object interactions in apraxia. Neuropsychologia 43, 917-929. https://doi.org/10.1016/j.neuropsychologia.2004.09.006

Byrge, L., Sporns, O., Smith, L.B., 2014. Developmental process emerges from extended brain-bodybehavior networks. Trends Cogn. Sci. 18, 395-403. https://doi.org/10.1016/j.tics.2014.04.010

Caldwell, C.A., 2020. Using experimental research designs to explore the scope of cumulative culture in humans and other animals. Top. Cogn. Sci. 12, 673-689. https://doi.org/10.1111/tops.12391

Caldwell, C.A., Millen, A.E., 2009. Social learning mechanisms and cumulative cultural evolution: Is imitation necessary? Psychol. Sci. 20, 1478-1483. https://doi.org/10.1111/j.14679280.2009.02469.x

Canzoneri, E., Ubaldi, S., Rastelli, V., Finisguerra, A., Bassolino, M., Serino, A., 2013. Tool-use reshapes the boundaries of body and peripersonal space representations. Exp. Brain Res. 228, 25-42. https://doi.org/10.1007/s00221-013-3532-2

Cardinali, L., Frassinetti, F., Brozzoli, C., Urquizar, C., Roy, A.C., Farnè, A., 2009. Tool-use induces morphological updating of the body schema. Curr. Biol. 19, R478-R479. https://doi.org/10.1016/j.cub.2009.05.009

Carello, C., Turvey, M., 2016. Dynamic (effortful) touch, in: Prescott, T., Ahissar, E., Izhikevich, E. (Eds.), Scholarpedia of Touch. Atlantis Press, Paris, pp. 227-240. https://doi.org/10.2991/978-946239-133-8_18

Carello, C., Wagman, J.B., 2009. Mutuality in the perception of affordances and the control of movement, in: Sternad, D. (Ed.), Progress in Motor Control. Springer, Boston, MA, pp. 273-292. https://doi.org/10.1007/978-0-387-77064-2_14

Caruana, F., Cuccio, V., 2017. Overcoming the acting/reasoning dualism in intelligent behavior. Phenomenol. Cogn. Sci. 16, 709-713. https://doi.org/10.1007/s11097-016-9471-1

Carver, N.S., Kelty-Stephen, D.G., 2017. Multifractality in individual honeybee behavior hints at colony-specific social cascades: Reanalysis of radio-frequency identification data from five different colonies. Phys. Rev. E 95, 22402. https://doi.org/10.1103/PhysRevE.95.022402

Chevalier-Skolnikoff, S., 1989. Spontaneous tool use and sensorimotor intelligence in Cebus compared with other monkeys and apes. Behav. Brain Sci. 12, 561-588. https://doi.org/10.1017/S0140525X00057678

Chisholm, R.L., Firtel, R.A., 2004. Insights into morphogenesis from a simple developmental system. Nat. Rev. Mol. Cell Biol. 5, 531-541. https://doi.org/10.1038/nrm1427

Cox, R.F.A., Smitsman, A.W., 2019. Action-selection perseveration in young children: Advances of a dynamic model. Dev. Psychobiol. 61, 43-55. https://doi.org/10.1002/dev.21776

Crain, B.J., Giray, T., Abramson, C.I., 2013. A tool for every job: Assessing the need for a universal definition of tool use. Int. J. Comp. Psychol. 26, 281-303. 
Crawford, L.E., Knouse, L.E., Kent, M., Vavra, D., Harding, O., LeServe, D., Fox, N., Hu, X., Li, P., Glory, C., Lambert, K.G., 2020. Enriched environment exposure accelerates rodent driving skills. Behav. Brain Res. 378, 112309. https://doi.org/10.1016/j.bbr.2019.112309

Curioni, A., Vesper, C., Knoblich, G., Sebanz, N., 2019. Reciprocal information flow and role distribution support joint action coordination. Cognition 187, 21-31. https://doi.org/10.1016/j.cognition.2019.02.006

Day, B., Ebrahimi, E., Hartman, L.S., Pagano, C.C., Babu, S. V, 2017. Calibration to tool use during visually-guided reaching. Acta Psychol. (Amst). 181, 27-39. https://doi.org/10.1016/j.actpsy.2017.09.014

Day, B., Ebrahimi, E., Hartman, L.S., Pagano, C.C., Robb, A.C., Babu, S. V, 2019. Examining the effects of altered avatars on perception-action in virtual reality. J. Exp. Psychol. Appl. 25, 1-24. https://doi.org/10.1037/xap0000192

de Guzman, M., Bird, G., Banissy, M.J., Catmur, C., 2016. Self-other control processes in social cognition: From imitation to empathy. Philos. Trans. R. Soc. B Biol. Sci. 371, 20150079. https://doi.org/10.1098/rstb.2015.0079

De Oliveira, E., Reynaud, E., Osiurak, F., 2019. Roles of technical reasoning, theory of mind, creativity, and fluid cognition in cumulative technological culture. Hum. Nat. 30, 326-340. https://doi.org/10.1007/s12110-019-09349-1

Derex, M., Bonnefon, J.-F., Boyd, R., Mesoudi, A., 2019. Causal understanding is not necessary for the improvement of culturally evolving technology. Nat. Hum. Behav. 3, 446-452. https://doi.org/10.1038/s41562-019-0567-9

Derex, M., Mesoudi, A., 2020. Cumulative cultural evolution within evolving population structures. Trends Cogn. Sci. 24, 654-667. https://doi.org/10.1016/j.tics.2020.04.005

Dinets, V., Brueggen, J.C., Brueggen, J.D., 2015. Crocodilians use tools for hunting. Ethol. Ecol. Evol. 27, 74-78. https://doi.org/10.1080/03949370.2013.858276

Dineva, E., Schöner, G., 2018. How infants' reaches reveal principles of sensorimotor decision making. Conn. Sci. 30, 53-80. https://doi.org/10.1080/09540091.2017.1405382

Dixon, M.L., De La Vega, A., Mills, C., Andrews-Hanna, J., Spreng, R.N., Cole, M.W., Christoff, K., 2018. Heterogeneity within the frontoparietal control network and its relationship to the default and dorsal attention networks. Proc. Natl. Acad. Sci. 115, E1598-E1607. https://doi.org/10.1073/pnas.1715766115

Doyon, J.K., Hajnal, A., Surber, T., Clark, J.D., Kelty-Stephen, D.G., 2019. Multifractality of posture modulates multisensory perception of stand-on-ability. PLoS One 14, e0212220. https://doi.org/10.1371/journal.pone.0212220

Dragovich, B., Mišić, N.Ž., 2019. p-Adic hierarchical properties of the genetic code. Biosystems 185, 104017. https://doi.org/10.1016/j.biosystems.2019.104017

Dusek, V., 2006. Philosophy of Technology: An Introduction. Blackwell, Malden, MA. 
1545 Emery, N.J., Clayton, N.S., 2009. Tool use and physical cognition in birds and mammals. Curr. Opin. Neurobiol. 19, 27-33. https://doi.org/10.1016/j.conb.2009.02.003

Fajen, B., 2013. Guiding locomotion in complex, dynamic environments. Front. Behav. Neurosci. 7, 85. https://doi.org/10.3389/fnbeh.2013.00085

Fajen, B.R., 2007. Affordance-based control of visually guided action. Ecol. Psychol. 19, 383-410. https://doi.org/10.1080/10407410701557877

1551

1552

1553

1554

1555

1556

1557

1558

1559

1560

1561

1562

1563

1564

1565

1566

1567

1568

1569

1570

1571

1572

1573

1574

1575

1576

1577

1578

1579

1580

1581

1582

Fajen, B.R., Matthis, J.S., 2011. Direct perception of action-scaled affordances: The shrinking gap problem. J. Exp. Psychol. Hum. Percept. Perform. 37, 1442-1457. https://doi.org/10.1037/a0023510

Falótico, T., Ottoni, E.B., 2016. The manifold use of pounding stone tools by wild capuchin monkeys of Serra da Capivara National Park, Brazil. Behaviour 153, 421-442. https://doi.org/10.1163/1568539X-00003357

Farnè, A., Iriki, A., Làdavas, E., 2005. Shaping multisensory action-space with tools: Evidence from patients with cross-modal extinction. Neuropsychologia 43, 238-248. https://doi.org/10.1016/j.neuropsychologia.2004.11.010

Fellers, J.H., Fellers, G.M., 1976. Tool use in a social insect and its implications for competitive interactions. Science (80-. ). 192, 70-72. https://doi.org/10.1126/science.192.4234.70

Fernandez-Gonzalez, R., Zallen, J.A., 2011. Oscillatory behaviors and hierarchical assembly of contractile structures in intercalating cells. Phys. Biol. 8, 45005. https://doi.org/10.1088/14783975/8/4/045005

Fink, P.W., Foo, P.S., Warren, W.H., 2009. Catching fly balls in virtual reality: A critical test of the outfielder problem. J. Vis. 9, 14. https://doi.org/10.1167/9.13.14

Finkel, L., Hogrefe, K., Frey, S.H., Goldenberg, G., Randerath, J., 2018. It takes two to pantomime: Communication meets motor cognition. Neurolmage Clin. 19, 1008-1017. https://doi.org/10.1016/j.nicl.2018.06.019

Finn, J.K., Tregenza, T., Norman, M.D., 2009. Defensive tool use in a coconut-carrying octopus. Curr. Biol. 19, R1069-R1070. https://doi.org/10.1016/j.cub.2009.10.052

Fischer, J., Mikhael, J.G., Tenenbaum, J.B., Kanwisher, N., 2016. Functional neuroanatomy of intuitive physical inference. Proc. Natl. Acad. Sci. 113, E5072-E5081. https://doi.org/10.1073/pnas.1610344113

Flynn, E.G., Laland, K.N., Kendal, R.L., Kendal, J.R., 2013. Target article with commentaries: Developmental niche construction. Dev. Sci. 16, 296-313. https://doi.org/10.1111/desc.12030

Fox, E.A., Sitampul, A.F., van Schaik, C.P., 1999. Intelligent tool use in wild Sumatran orangutans, in: Parke, S.T., Mitchell, R.W., Miles, L.H. (Eds.), The Mentality of Gorillas and Orangutans. Cambridge University Press, Cambridge, UK, pp. 99-116.

Fragaszy, D.M., Biro, D., Eshchar, Y., Humle, T., Izar, P., Resende, B., Visalberghi, E., 2013. The fourth dimension of tool use: Temporally enduring artefacts aid primates learning to use tools. Philos. Trans. R. Soc. Lond. B. Biol. Sci. 368, 20120410. https://doi.org/10.1098/rstb.2012.0410 
1583

1584

1585

1586

1587

1588

1589

1590

1591

1592

1593

1594

1595

1596

1597

1598

1599

1600

1601

1602

1603

1604

1605

1606

1607

1608

1609

1610

1611

1612

1613

1614

1615

1616

1617

1618

1619

Fragaszy, D.M., Kuroshima, H., Stone, B.W., 2015. "Vision for action” in young children aligning multifeatured objects: Development and comparison with nonhuman primates. PLoS One 10, e0140033. https://doi.org/10.1371/journal.pone.0140033

Fragaszy, D.M., Mangalam, M., 2018. Tooling. Adv. Study Behav. 50, 177-241. https://doi.org/10.1016/bs.asb.2018.01.001

Fragaszy, D.M., Stone, B.W., Scott, N.M., Menzel, C., 2011. How tufted capuchin monkeys (Cebus apella spp) and common chimpanzees (Pan troglodytes) align objects to surfaces: Insights into spatial reasoning and implications for tool use. Am. J. Primatol. 73, 1012-1030. https://doi.org/10.1002/ajp.20966

Fratzl, P, 2008. Collagen: Structure and mechanics, an Introduction, in: Fratzl, Peter (Ed.), Collagen. Springer US, Boston, MA, pp. 1-13. https://doi.org/10.1007/978-0-387-73906-9_1

Galef, B.G., 2013. Imitation and local enhancement: Detrimental effects of consensus definitions on analyses of social learning in animals. Behav. Processes 100, 123-130. https://doi.org/10.1016/j.beproc.2013.07.026

Gallivan, J.P., McLean, A., Culham, J.C., 2011. Neuroimaging reveals enhanced activation in a reachselective brain area for objects located within participants' typical hand workspaces. Neuropsychologia 49, 3710-3721. https://doi.org/10.1016/j.neuropsychologia.2011.09.027

Gallivan, J.P., McLean, D.A., Valyear, K.F., Culham, J.C., 2013. Decoding the neural mechanisms of human tool use. Elife 2, e00425. https://doi.org/10.7554/eLife.00425

Gavelis, G.S., Wakeman, K.C., Tillmann, U., Ripken, C., Mitarai, S., Herranz, M., Özbek, S., Holstein, T., Keeling, P.J., Leander, B.S., 2017. Microbial arms race: Ballistic "nematocysts" in dinoflagellates represent a new extreme in organelle complexity. Sci. Adv. 3, e1602552. https://doi.org/10.1126/sciadv.1602552

Gentner, D., Hoyos, C., 2017. Analogy and abstraction. Top. Cogn. Sci. 9, 672-693. https://doi.org/10.1111/tops.12278

Gerullis, P., Schuster, S., 2014. Archerfish actively control the hydrodynamics of their jets. Curr. Biol. 24, 2156-2160. https://doi.org/10.1016/j.cub.2014.07.059

Gibson, J.J., 1979. The Ecological Approach to Visual Perception. Houghton Mifflin, Boston, MA.

Gibson, J.J., 1966. The Senses Considered as Perceptual Systems. Houghton Mifflin, Boston, MA.

Gibson, K.R., Ingold, T., 1994. Tools, Language and Cognition in Human Evolution. Cambridge University Press, Cambridge, UK.

Gobet, F., 2015. Understanding Expertise: A Multi-disciplinary Approach. Macmillan International Higher Education.

Goldberger, A.L., Rigney, D.R., West, B.J., 1990. Chaos and fractals in human physiology. Sci. Am. 262, 42-49.

Goldenberg, G., Hagmann, S., 1998. Tool use and mechanical problem solving in apraxia. Neuropsychologia 36, 581-589. https://doi.org/10.1016/S0028-3932(97)00165-6 
Goldenberg, G., Hartmann-Schmid, K., Sürer, F., Daumüller, M., Hermsdölrfer, J., 2007. The impact of dysexecutive syndrome on use of tools and technical devices. Cortex 43, 424-435. https://doi.org/10.1016/S0010-9452(08)70467-2

Goldenberg, G., Spatt, J., 2009. The neural basis of tool use. Brain 132, 1645-1655. https://doi.org/10.1093/brain/awp080

Golonka, S., Wilson, A.D., 2019. Ecological representations. Ecol. Psychol. 31, 235-253. https://doi.org/10.1080/10407413.2019.1615224

Gönül, G., Takmaz, E.K., Hohenberger, A., Corballis, M., 2018. The cognitive ontogeny of tool making in children: The role of inhibition and hierarchical structuring. J. Exp. Child Psychol. 173, 222 238. https://doi.org/10.1016/j.jecp.2018.03.017

Gowlland, G., 2019. The sociality of enskilment. Ethnos 84, 508-524. https://doi.org/10.1080/00141844.2018.1455726

Gruber, M.J., Ranganath, C., 2019. How curiosity enhances hippocampus-dependent memory: The prediction, appraisal, curiosity, and exploration (PACE) framework. Trends Cogn. Sci. 23, 10141025. https://doi.org/10.1016/j.tics.2019.10.003

Haar, S., van Assel, C.M., Faisal, A.A., 2020. Motor learning in real-world pool billiards. Sci. Rep. 10, 20046. https://doi.org/10.1038/s41598-020-76805-9

Haggard, P., 2017. Sense of agency in the human brain. Nat. Rev. Neurosci. 18, 196-207. https://doi.org/10.1038/nrn.2017.14

Hajnal, A., Clark, J.D., Doyon, J.K., Kelty-Stephen, D.G., 2018. Fractality of body movements predicts perception of affordances: Evidence from stand-on-ability judgments about slopes. J. Exp. Psychol. Hum. Percept. Perform. 44, 836-841. https://doi.org/10.1037/xhp0000510

Hajnal, A., Fonseca, S., Harrison, S., Kinsella-Shaw, J.M., Carello, C., 2007. Comparison of dynamic (effortful) touch by hand and foot. J. Mot. Behav. 39, 82-88. https://doi.org/10.3200/JMBR.39.2.82-88

Hamilton, W.J., Buskirk, R.E., Buskirk, W.H., 1975. Defensive stoning of baboons. Nature 256, 488489. https://doi.org/10.1038/256488a0

Hansell, M., Ruxton, G.D., 2008. Setting tool use within the context of animal construction behaviour. Trends Ecol. Evol. 23, 73-78. https://doi.org/10.1016/j.tree.2007.10.006

Hardus, M.E., Lameira, A.R., van Schaik, C.P., Wich, S.A., 2009. Tool use in wild orangutans modifies sound production: A functionally deceptive innovation? Proc. R. Soc. London B Biol. Sci. 276, 3689-3694. https://doi.org/10.1098/rspb.2009.1027

Harrison, S.J., Hajnal, A., Lopresti-Goodman, S., Isenhower, R.W., Kinsella-Shaw, J.M., 2011. Perceiving action-relevant properties of tools through dynamic touch: Effects of mass distribution, exploration style, and intention. J. Exp. Psychol. Hum. Percept. Perform. 37, 193-206. https://doi.org/10.1037/a0020407 
1656

Harrison, S.J., Reynolds, N., Bishoff, B., Stergiou, N., 2021. Assessing the relative contribution of vision to odometry via manipulations of gait in an over-ground homing task. Exp. Brain Res. https://doi.org/10.1007/s00221-021-06066-z

Harrison, S.J., Stergiou, N., 2015. Complex adaptive behavior and dexterous action. Nonlinear Dynamics. Psychol. Life Sci. 19, 345-394.

Hart, B.L., Hart, L.A., McCoy, M., Sarath, C.R., 2001. Cognitive behaviour in Asian elephants: Use and modification of branches for fly switching. Anim. Behav. 62, 839-847. https://doi.org/10.1006/anbe.2001.1815

Hartman, L.S., Kil, I., Pagano, C.C., Burg, T., 2016. Investigating haptic distance-to-break using linear and nonlinear materials in a simulated minimally invasive surgery task. Ergonomics 59, 11711181. https://doi.org/10.1080/00140139.2015.1127429

Haslam, M., 2013. 'Captivity bias' in animal tool use and its implications for the evolution of hominin technology. Philos. Trans. R. Soc. B Biol. Sci. 368, 20120421. https://doi.org/10.1098/rstb.2012.0421

Hasson, U., Frith, C.D., 2016. Mirroring and beyond: coupled dynamics as a generalized framework for modelling social interactions. Philos. Trans. R. Soc. B Biol. Sci. 371, 20150366. https://doi.org/10.1098/rstb.2015.0366

Hayakawa, Y., Ariyama, H., Stancikova, J., Sakitani, K., Asfaha, S., Renz, B.W., Dubeykovskaya, Z.A., Shibata, W., Wang, H., Westphalen, C.B., Chen, X., Takemoto, Y., Kim, W., Khurana, S.S., Tailor, Y., Nagar, K., Tomita, H., Hara, A., Sepulveda, A.R., Setlik, W., Gershon, M.D., Saha, S., Ding, L., Shen, Z., Fox, J.G., Friedman, R.A., Konieczny, S.F., Worthley, D.L., Korinek, V., Wang, T.C., 2015. Mist1 expressing gastric stem cells maintain the normal and neoplastic gastric epithelium and are supported by a perivascular stem cell niche. Cancer Cell 28, 800-814. https://doi.org/10.1016/j.ccell.2015.10.003

Heald, J.B., Ingram, J.N., Flanagan, J.R., Wolpert, D.M., 2018. Multiple motor memories are learned to control different points on a tool. Nat. Hum. Behav. 2, 300-311. https://doi.org/10.1038/s41562018-0324-5

Hecht, E.E., Gutman, D.A., Khreisheh, N., Taylor, S. V, Kilner, J., Faisal, A.A., Bradley, B.A., Chaminade, T., Stout, D., 2015. Acquisition of Paleolithic toolmaking abilities involves structural remodeling to inferior frontoparietal regions. Brain Struct. Funct. 220, 2315-2331. https://doi.org/10.1007/s00429-014-0789-6

Heilman, K.M., Rothi, L.J., Valenstein, E., 1982. Two forms of ideomotor apraxia. Neurology 32, $342-$ 342. https://doi.org/10.1212/WNL.32.4.342

Hermsdörfer, J., Li, Y., Randerath, J., Goldenberg, G., Johannsen, L., 2012. Tool use without a tool: Kinematic characteristics of pantomiming as compared to actual use and the effect of brain damage. Exp. Brain Res. 218, 201-214. https://doi.org/10.1007/s00221-012-3021-z

Hermsdörfer, J., Terlinden, G., Mühlau, M., Goldenberg, G., Wohlschläger, A.M., 2007. Neural representations of pantomimed and actual tool use: Evidence from an event-related fMRI study. Neuroimage 36, T109-T118. https://doi.org/10.1016/j.neuroimage.2007.03.037 
1695

1696

1697

1698

1699

1700

1701

1702

1703

1704

1705

1706

1707

1708

1709

1710

1711

1712

1713

1714

1715

1716

1717

1718

1719

1720

1721

1722

1723

1724

1725

1726

1727

1728

1729

1730

Heuer, H., Sülzenbrück, S., 2009. Trajectories in operating a handheld tool. J. Exp. Psychol. Hum. Percept. Perform. 35, 375-389. https://doi.org/10.1037/0096-1523.35.2.375

Heyes, C., 2016. Imitation: Not in our genes. Curr. Biol. 26, R412-R414. https://doi.org/10.1016/j.cub.2016.03.060

Heyes, C.M., 2018. Cognitive Gadgets: The Cultural Evolution of Thinking. Cambridge University Press, Cambridge, MA.

Higuchi, T., Cinelli, M.E., Greig, M.A., Patla, A.E., 2006. Locomotion through apertures when wider space for locomotion is necessary: Adaptation to artificially altered bodily states. Exp. Brain Res. 175, 50-59. https://doi.org/10.1007/s00221-006-0525-4

Hill, K., Barton, M., Hurtado, A.M., 2009. The emergence of human uniqueness: Characters underlying behavioral modernity. Evol. Anthropol. Issues, News, Rev. 18, 187-200. https://doi.org/10.1002/evan.20224

Hodges, J.R., Bozeat, S., Ralph, M.A.L., Patterson, K., Spatt, J., 2000. The role of conceptual knowledge in object use Evidence from semantic dementia. Brain 123, 1913-1925. https://doi.org/10.1093/brain/123.9.1913

Huber, L., Gajdon, G.K., 2006. Technical intelligence in animals: The kea model. Anim. Cogn. 9, 295305. https://doi.org/10.1007/s10071-006-0033-8

Hughes, T.P., 1987. The evolution of large technological systems, in: The Social Construction of Technological Systems: New Directions in the Sociology and History of Technologyechnology. MIT Press, Cambridge, MA.

Hunt, G.R., 1996. Manufacture and use of hook-tools by New Caledonian crows. Nature 379, 249 251. https://doi.org/10.1038/379249a0

Hunt, G.R., Gray, R.D., Taylor, A.H., 2013. Why is tool use rare in animals, in: Sanz, C., Call, J., Boesch, C. (Eds.), Tool Use in Animals: Cognition and Ecology. Cambridge University Press, Cambridge, UK, pp. 89-118.

Ingber, D.E., 2006. Cellular mechanotransduction: Putting all the pieces together again. FASEB J. 20, 811-827. https://doi.org/10.1096/fj.05-5424rev

Ingmanson, E.J., 1996. Tool-using behavior in wild Pan paniscus: Social and ecological considerations, in: Russon, A.R., Bard, K.A., Parker, S.T. (Eds.), Reaching into Thought: The Minds of the Great Apes. Cambridge University Press, New York, NY, pp. 190-210.

Ingram, J.N., Howard, I.S., Flanagan, J.R., Wolpert, D.M., 2010. Multiple grasp-specific representations of tool dynamics mediate skillful manipulation. Curr. Biol. 20, 618-623. https://doi.org/10.1016/j.cub.2010.01.054

Iriki, A., Tanaka, M., Iwamura, Y., 1996. Attention-induced neuronal activity in the monkey somatosensory cortex revealed by pupillometrics. Neurosci. Res. 25, 173-181. https://doi.org/10.1016/0168-0102(96)01043-7 
Jacobs, S., Bussel, B., Combeaud, M., Roby-Brami, A., 2009. The use of a tool requires its incorporation into the movement: Evidence from stick-pointing in apraxia. Cortex 45, 444-455. https://doi.org/10.1016/j.cortex.2007.12.009

Johnson-Frey, S.H., 2004. The neural bases of complex tool use in humans. Trends Cogn. Sci. 8, 7178. https://doi.org/10.1016/j.tics.2003.12.002

Johnson-Frey, S.H., 2003. What's so special about human tool use? Neuron 39, 201-204. https://doi.org/10.1016/S0896-6273(03)00424-0

Kadar, E.E., Shaw, R.E., 2000. Toward an ecological field theory of perceptual control of locomotion. Ecol. Psychol. 12, 141-180. https://doi.org/10.1207/S15326969ECO1202_02

Kahrs, B.A., Lockman, J.J., 2014. Tool using. Child Dev. Perspect. 8, 231-236. https://doi.org/10.1111/cdep.12087

Kaplan, H., Gurven, M., Winking, J., Hooper, P.L., Stieglitz, J., 2010. Learning, menopause, and the human adaptive complex. Ann. N. Y. Acad. Sci. 1204, 30-42. https://doi.org/10.1111/j.17496632.2010.05528.x

Kelso, J.A.S., 2009. Synergies: Atoms of brain and behavior. Prog. Mot. Control 83-91. https://doi.org/10.1007/978-0-387-77064-2_5

Kelso, J.S., Tuller, B., Vatikiotis-Bateson, E., Fowler, C.A., 1984. Functionally specific articulatory cooperation following jaw perturbations during speech: Evidence for coordinative structures. J. Exp. Psychol. Hum. Percept. Perform. 10, 812-832. https://doi.org/10.1037/0096-1523.10.6.812

Kelty-Stephen, D.G., Dixon, J.A., 2014. Interwoven fluctuations during intermodal perception: Fractality in head sway supports the use of visual feedback in haptic perceptual judgments by manual wielding. J. Exp. Psychol. Hum. Percept. Perform. 40, 2289-2309. https://doi.org/10.1037/a0038159

Kendal, R.L., Boogert, N.J., Rendell, L., Laland, K.N., Webster, M., Jones, P.L., 2018. Social learning strategies: Bridge-building between fields. Trends Cogn. Sci. 22, 651-665. https://doi.org/10.1016/j.tics.2018.04.003

Kennedy, D.P., D’Onofrio, B.M., Quinn, P.D., Bölte, S., Lichtenstein, P., Falck-Ytter, T., 2017. Genetic influence on eye movements to complex scenes at short timescales. Curr. Biol. 27, 35543560.e3. https://doi.org/10.1016/j.cub.2017.10.007

Kianianmomeni, A., Hallmann, A., 2014. Algal photoreceptors: In vivo functions and potential applications. Planta 239, 1-26. https://doi.org/10.1007/s00425-013-1962-5

Kline, M.A., 2015. How to learn about teaching: An evolutionary framework for the study of teaching behavior in humans and other animals. Behav. Brain Sci. 38, e31. https://doi.org/10.1017/S0140525X14000090

Köhler, W., 1925. The Mentality of Apes. Vintage Books, New York, NY.

Koster, J., McElreath, R., Hill, K., Yu, D., Shepard, G., van Vliet, N., Gurven, M., Trumble, B., Bird, R.B., Bird, D., Codding, B., Coad, L., Pacheco-Cobos, L., Winterhalder, B., Lupo, K., Schmitt, D., Sillitoe, P., Franzen, M., Alvard, M., Venkataraman, V., Kraft, T., Endicott, K., Beckerman, S., 
Marks, S.A., Headland, T., Pangau-Adam, M., Siren, A., Kramer, K., Greaves, R., Reyes-García, V., Guèze, M., Duda, R., Fernández-Llamazares, Á., Gallois, S., Napitupulu, L., Ellen, R., Ziker, J., Nielsen, M.R., Ready, E., Healey, C., Ross, C., 2020. The life history of human foraging: Cross-cultural and individual variation. Sci. Adv. 6, eaax9070. https://doi.org/10.1126/sciadv.aax9070

Krakauer, J.W., Ghazanfar, A.A., Gomez-Marin, A., Maclver, M.A., Poeppel, D., 2017. Neuroscience needs behavior: Correcting a reductionist bias. Neuron 93, 480-490. https://doi.org/10.1016/j.neuron.2016.12.041

Króliczak, G., Frey, S.H., 2009. A common network in the left cerebral hemisphere represents planning of tool use pantomimes and familiar intransitive gestures at the hand-independent level. Cereb. Cortex 19, 2396-2410. https://doi.org/10.1093/cercor/bhn261

la Cour, L.T., Stone, B.W., Hopkins, W., Menzel, C., Fragaszy, D.M., 2014. What limits tool use in nonhuman primates? Insights from tufted capuchin monkeys (Sapajus spp.) and chimpanzees (Pan troglodytes) aligning three-dimensional objects to a surface. Anim. Cogn. 17, 113-125. https://doi.org/10.1007/s10071-013-0643-x

Ladouce, S., Donaldson, D.I., Dudchenko, P.A., letswaart, M., 2019. Mobile EEG identifies the reallocation of attention during real-world activity. Sci. Rep. 9, 15851. https://doi.org/10.1038/s41598-019-51996-y

Laland, K.N., Uller, T., Feldman, M.W., Sterelny, K., Müller, G.B., Moczek, A., Jablonka, E., OdlingSmee, J., 2015. The extended evolutionary synthesis: Its structure, assumptions and predictions. Proc. R. Soc. B Biol. Sci. 282, 20151019. https://doi.org/10.1098/rspb.2015.1019

Latash, M.L., Scholz, J.P., Schöner, G., 2007. Toward a new theory of motor synergies. Motor Control 11, 276-308. https://doi.org/10.1123/mcj.11.3.276

Lawick-Goodall, J. Van, 1971. Tool-using in primates and other vertebrates. Adv. Study Behav. 3, 195249. https://doi.org/10.1016/S0065-3454(08)60157-6

Legare, C.H., Nielsen, M., 2015. Imitation and innovation: The dual engines of cultural learning. Trends Cogn. Sci. 19, 688-699. https://doi.org/10.1016/j.tics.2015.08.005

Lesourd, M., Baumard, J., Jarry, C., Etcharry-Bouyx, F., Belliard, S., Moreaud, O., Croisile, B., Chauviré, V., Granjon, M., Le Gall, D., Osiurak, F., 2016. Mechanical problem-solving strategies in Alzheimer's disease and semantic dementia. Neuropsychology 30, 612-623. https://doi.org/10.1037/neu0000241

Lewis, J.W., 2006. Cortical networks related to human use of tools. Neurosci. 12, 211-231. https://doi.org/10.1177/1073858406288327

Lockman, J.J., 2005. Tool use from a perception-action perspective: Developmental and evolutionary considerations, in: Roux, V., Bril, B. (Eds.), Stone Knapping: The Necessary Conditions for a Uniquely Hominid Behaviour. McDonald Institute for Archaeological Research, Cambridge, UK, pp. 313-330. 
1806

1807

1808

1809

1810

1811

1812

1813

1814

1815

1816

1817

1818

1819

1820

1821

1822

1823

1824

1825

1826

1827

1828

1829

1830

1831

1832

1833

1834

1835

1836

1837

1838

1839

1840

1841

1842

Long, L.O., Pagano, C.C., Singapogu, R.B., Burg, T.C., 2016. Surgeon's perception of soft tissue constraints and distance-to-break in a simulated minimally invasive surgery task. Proc. Hum. Factors Ergon. Soc. Annu. Meet. 60, 1600-1604. https://doi.org/10.1177/1541931213601369

Lundberg, D.S., Lebeis, S.L., Paredes, S.H., Yourstone, S., Gehring, J., Malfatti, S., Tremblay, J., Engelbrektson, A., Kunin, V., Rio, T.G. del, Edgar, R.C., Eickhorst, T., Ley, R.E., Hugenholtz, P., Tringe, S.G., Dangl, J.L., 2012. Defining the core Arabidopsis thaliana root microbiome. Nature 488, 86-90. https://doi.org/10.1038/nature11237

Mandelbrot, B., 1967. How long is the coast of britain? Statistical self-similarity and fractional dimension. Science (80-. ). 156, 636-638. https://doi.org/10.1126/science.156.3775.636

Mandelbrot, B.B., 2013. Multifractals and 1/f Noise; Wild Self-Affinity in Physics. Springer Verlag, New York, NY.

Mangalam, M., 2016. What makes a tool, in: Weekes-Shackelford, V., Shackelford, T.K., WeekesShackelford, V.A. (Eds.), Encyclopedia of Evolutionary Psychological Science. Springer, New York, NY, pp. 1-5. https://doi.org/10.1007/978-3-319-16999-6_3153-1

Mangalam, M., Barton, S.A., Wagman, J.B., Fragaszy, D.M., Newell, K.M., 2017. Perception of the length of an object through dynamic touch is invariant across changes in the medium. Attention, Perception, Psychophys. 79, 2499-2509. https://doi.org/10.3758/s13414-017-1403-9

Mangalam, Madhur, Carver, N.S., Kelty-Stephen, D.G., 2020a. Multifractal signatures of perceptual processing on anatomical sleeves of the human body. J. R. Soc. Interface 17, 20200328. https://doi.org/10.1098/rsif.2020.0328

Mangalam, Madhur, Carver, N.S., Kelty-Stephen, D.G., 2020b. Global broadcasting of local fractal fluctuations in a bodywide distributed system supports perception via effortful touch. Chaos, Solitons \& Fractals 135, 109740. https://doi.org/10.1016/j.chaos.2020.109740

Mangalam, Madhur, Chen, R., McHugh, T.R., Singh, T., Kelty-Stephen, D.G., 2020c. Bodywide fluctuations support manual exploration: Fractal fluctuations in posture predict perception of heaviness and length via effortful touch by the hand. Hum. Mov. Sci. 69, 102543. https://doi.org/10.1016/j.humov.2019.102543

Mangalam, M., Conners, J.D., Kelty-Stephen, D.G., Singh, T., 2019a. Fractal fluctuations in muscular activity contribute to judgments of length but not heaviness via dynamic touch. Exp. Brain Res. 237, 1213-1216. https://doi.org/10.1007/s00221-019-05505-2

Mangalam, M., Conners, J.D., Singh, T., 2019b. Muscular effort differentially mediates perception of heaviness and length via dynamic touch. Exp. Brain Res. 237, 237-246. https://doi.org/10.1007/s00221-018-5421-1

Mangalam, M., Fragaszy, D.M., 2016. Transforming the body-only system into the body-plus-tool system. Anim. Behav. 117, 115-122. https://doi.org/10.1016/j.anbehav.2016.04.016

Mangalam, M., Fragaszy, D.M., 2015. Wild bearded capuchin monkeys crack nuts dexterously. Curr. Biol. 25, 1334-1339. https://doi.org/10.1016/j.cub.2015.03.035 
Mangalam, M., Izar, P., Visalberghi, E., Fragaszy, D.M., 2016. Task-specific temporal organization of percussive movements in wild bearded capuchin monkeys. Anim. Behav. 114, 129-137. https://doi.org/10.1016/j.anbehav.2016.01.011

Mangalam, M., Kelty-Stephen, D.G., 2020. Multiplicative-cascade dynamics supports whole-body coordination for perception via effortful touch. Hum. Mov. Sci. 70, 102595. https://doi.org/10.1016/j.humov.2020.102595

Mangalam, M., Pacheco, M.M., Fragaszy, D.M., Newell, K.M., 2019c. Perceptual learning of tooling affordances of a jointed object via dynamic touch. Ecol. Psychol. 31, 14-29. https://doi.org/10.1080/10407413.2018.1473714

Mangalam, M., Pacheco, M.M., Izar, P., Visalberghi, E., Fragaszy, D.M., 2018a. Unique perceptuomotor control of stone hammers in wild monkeys. Biol. Lett. 14, 20170587. https://doi.org/10.1098/rsbl.2017.0587

Mangalam, M., Rein, R., Fragaszy, D.M., 2018b. Bearded capuchin monkeys use joint synergies to stabilize the hammer trajectory while cracking nuts in bipedal stance. Proc. R. Soc. B Biol. Sci. 285, 20181797. https://doi.org/10.1098/rspb.2018.1797

Mangalam, M., Roles, L.K.R., Fragaszy, D.M., 2020. Distinct perceptuomotor features of percussive tooling in humans (Homo sapiens) and wild bearded capuchin monkeys (Sapajus libidinosus). J. Comp. Psychol. 134, 84-97. https://doi.org/10.1037/com0000197

Maravita, A., Husain, M., Clarke, K., Driver, J., 2001. Reaching with a tool extends visual-tactile interactions into far space: evidence from cross-modal extinction. Neuropsychologia 39, 580-585. https://doi.org/10.1016/S0028-3932(00)00150-0

Maravita, A., Iriki, A., 2004. Tools for the body (schema). Trends Cogn. Sci. 8, 79-86. https://doi.org/10.1016/j.tics.2003.12.008

Margulies, D.S., Ghosh, S.S., Goulas, A., Falkiewicz, M., Huntenburg, J.M., Langs, G., Bezgin, G., Eickhoff, S.B., Castellanos, F.X., Petrides, M., Jefferies, E., Smallwood, J., 2016. Situating the default-mode network along a principal gradient of macroscale cortical organization. Proc. Natl. Acad. Sci. 113, 12574-12579. https://doi.org/10.1073/pnas.1608282113

Martel, M., Cardinali, L., Roy, A.C., Farnè, A., 2016. Tool-use: An open window into body representation and its plasticity. Cogn. Neuropsychol. 33, 82-101. https://doi.org/10.1080/02643294.2016.1167678

Martin, M., Beume, L., Kümmerer, D., Schmidt, C.S.M., Bormann, T., Dressing, A., Ludwig, V.M., Umarova, R.M., Mader, I., Rijntjes, M., Kaller, C.P., Weiller, C., 2016a. Differential roles of ventral and dorsal streams for conceptual and production-related components of tool use in acute stroke patients. Cereb. Cortex 26, 3754-3771. https://doi.org/10.1093/cercor/bhv179

Martin, M., Nitschke, K., Beume, L., Dressing, A., Bühler, L.E., Ludwig, V.M., Mader, I., Rijntjes, M., Kaller, C.P., Weiller, C., 2016b. Brain activity underlying tool-related and imitative skills after major left hemisphere stroke. Brain 139, 1497-1516. https://doi.org/10.1093/brain/aww035 
1880

1881

1882

1883

1884

1885

1886

1887

1888

1889

1890

1891

1892

1893

1894

1895

1896

1897

1898

1899

1900

1901

1902

1903

1904

1905

1906

1907

1908

1909

1910

1911

1912

1913

1914

1915

1916

Martinho, A., Burns, Z.T., von Bayern, A.M.P., Kacelnik, A., 2014. Monocular tool control, eye dominance, and laterality in New Caledonian crows. Curr. Biol. 24, 2930-2934. https://doi.org/10.1016/j.cub.2014.10.035

Mather, J.A., 1994. "Home" choice and modification by juvenile Octopus vulgaris (Mollusca: Cephalopoda): Specialized intelligence and tool use? J. Zool. 233, 359-368. https://doi.org/10.1111/j.1469-7998.1994.tb05270.x

Mathieu, J.E., Hollenbeck, J.R., van Knippenberg, D., Ilgen, D.R., 2017. A century of work teams in the Journal of Applied Psychology. J. Appl. Psychol. 102, 452-467. https://doi.org/10.1037/apl0000128

Matsuzawa, Tetsuro, 2001. Primate foundations of human intelligence: A view of tool use in nonhuman primates and fossil hominids, in: Matsuzawa, T (Ed.), Primate Origins of Human Cognition and Behavior. Springer-Verlag, Tokyo, Japan, pp. 3-25.

Matusz, P.J., Dikker, S., Huth, A.G., Perrodin, C., 2019. Are we ready for real-world neuroscience? J. Cogn. Neurosci. 31, 327-338. https://doi.org/10.1162/jocn_e_01276

McDougle, S.D., Ivry, R.B., Taylor, J.A., 2016. Taking aim at the cognitive side of learning in sensorimotor adaptation tasks. Trends Cogn. Sci. 20, 535-544. https://doi.org/10.1016/j.tics.2016.05.002

McNamee, D., Wolpert, D.M., 2019. Internal models in biological control. Annu. Rev. Control. Robot. Auton. Syst. 2, 339-364. https://doi.org/10.1146/annurev-control-060117-105206

Mengotti, P., Corradi-Dell'Acqua, C., Negri, G.A.L., Ukmar, M., Pesavento, V., Rumiati, R.I., 2013. Selective imitation impairments differentially interact with language processing. Brain 136, 2602 2618. https://doi.org/10.1093/brain/awt194

Mesoudi, A., 2021. Blind and incremental or directed and disruptive? On the nature of novel variation in human cultural evolution. Am. Philos. Q. 58, 7-20. https://doi.org/10.2307/48600682

Mesoudi, A., Thornton, A., 2021. What is cumulative cultural evolution? Proc. R. Soc. B Biol. Sci. 285, 20180712. https://doi.org/10.1098/rspb.2018.0712

Michaels, C., Carello, C., 2000. Direct Perception. Prentice-Hall, Englewood Cliffs, NJ.

Michaels, C.F., Weier, Z., Harrison, S.J., 2007. Using vision and dynamic touch to perceive the affordances of tools. Perception 36, 750-772. https://doi.org/10.1068/p5593

Michener, G.R., 2004. Hunting techniques and tool use by North American badgers preying on Richardson's ground squirrels. J. Mammal. 85, 1019-1027. https://doi.org/10.1644/BNS-102

Miller, L.E., Montroni, L., Koun, E., Salemme, R., Hayward, V., Farnè, A., 2018. Sensing with tools extends somatosensory processing beyond the body. Nature 561, 239-242. https://doi.org/10.1038/s41586-018-0460-0

Miu, E., Gulley, N., Laland, K.N., Rendell, L., 2020. Flexible learning, rather than inveterate innovation or copying, drives cumulative knowledge gain. Sci. Adv. 6, eaaz0286. https://doi.org/10.1126/sciadv.aaz0286 
Morgan, T.J.H., Uomini, N.T., Rendell, L.E., Chouinard-Thuly, L., Street, S.E., Lewis, H.M., Cross, C.P., Evans, C., Kearney, R., de la Torre, I., Whiten, A., Laland, K.N., 2015. Experimental evidence for the co-evolution of hominin tool-making teaching and language. Nat. Commun. 6, 6029. https://doi.org/10.1038/ncomms7029

Newell, K.M., 1986. Constraints on the development of coordination, in: Wade, M.G., Whiting, H.T. (Eds.), Motor Development in Children: Aspects of Coordination and Control. Martinus Nijhoff, Dordrecht, Netherlands, pp. 341-360.

Newell, K.M., Jordan, K., 2007. Task constraints and movement organization: A common language, in: Broadhead, G.D., Davis, W.E. (Eds.), Ecological Task Analysis and Movement. Human Kinetics, Champaign, IL, pp. 5-23.

Newen, A., De Bruin, L., Gallagher, S., 2018. The Oxford Handbook of 4E Cognition. Oxford University Press, Oxford, UK.

Norman, J., 2002. Two visual systems and two theories of perception: An attempt to reconcile the constructivist and ecological approaches. Behav. Brain Sci. 25, 73-96. https://doi.org/DOI: $10.1017 /$ S0140525X0200002X

Onnela, J.-P., 2021. Opportunities and challenges in the collection and analysis of digital phenotyping data. Neuropsychopharmacology 46, 45-54. https://doi.org/10.1038/s41386-020-0771-3

Orban, G.A., Caruana, F., 2014. The neural basis of human tool use. Front. Psychol. 5, 310. https://doi.org/10.3389/fpsyg.2014.00310

Osiurak, F., 2020. The Tool Instinct. John Wiley \& Sons.

Osiurak, F., 2017. Cognitive paleoanthropology and technology: Toward a parsimonious theory (PATH). Rev. Gen. Psychol. 21, 292-307. https://doi.org/10.1037/gpr0000129

Osiurak, F., 2014. What neuropsychology tells us about human tool use? The four constraints theory (4CT): Mechanics, space, time, and effort. Neuropsychol. Rev. 24, 88-115. https://doi.org/10.1007/s11065-014-9260-y

Osiurak, F., Aubin, G., Allain, P., Jarry, C., Richard, I., Le Gall, D., 2008. Object utilization and object usage: A single-case study. Neurocase 14, 169-183. https://doi.org/10.1080/13554790802108372

Osiurak, F., Badets, A., 2016. Tool use and affordance: Manipulation-based versus reasoning-based approaches. Psychol. Rev. 123, 534-568. https://doi.org/10.1037/rev0000027

Osiurak, F., Cretel, C., Duhau-Marmon, N., Fournier, I., Marignier, L., De Oliveira, E., Navarro, J., Reynaud, E., 2021a. The pedagogue, the engineer, and the friend: From whom do we learn? Nat. Hum. Behav. In press.

Osiurak, F., De Oliveira, E., Navarro, J., Lesourd, M., Claidière, N., Reynaud, E., 2016. Physical intelligence does matter to cumulative technological culture. J. Exp. Psychol. Gen. 145, 941-948. https://doi.org/10.1037/xge0000189 
1953

1954

1955

1956

1957

1958

1959

1960

1961

1962

1963

1964

1965

1966

1967

1968

1969

1970

1971

1972

1973

1974

1975

1976

1977

1978

1979

1980

1981

1982

1983

1984

1985

1986

1987

1988

Osiurak, F., De Oliveira, E., Navarro, J., Reynaud, E., 2020a. The castaway island: Distinct roles of theory of mind and technical reasoning in cumulative technological culture. J. Exp. Psychol. Gen. 149, 58-66. https://doi.org/10.1037/xge0000614

Osiurak, F., Federico, G., 2020. Four ways of (mis-)conceiving embodiment in tool use. Synthese. https://doi.org/10.1007/s11229-020-02960-1

Osiurak, F., Heinke, D., 2018. Looking for intoolligence: A unified framework for the cognitive study of human tool use and technology. Am. Psychol. 73, 169-185. https://doi.org/10.1037/amp0000162

Osiurak, F., Jarry, C., Allain, P., Aubin, G., Etcharry-Bouyx, F., Richard, I., Bernard, I., Le Gall, D., 2009. Unusual use of objects after unilateral brain damage. The technical reasoning model. Cortex 45, 769-783. https://doi.org/10.1016/j.cortex.2008.06.013

Osiurak, F., Jarry, C., Le Gall, D., 2010. Grasping the affordances, understanding the reasoning: Toward a dialectical theory of human tool use. Psychol. Rev. 117, 517-540. https://doi.org/10.1037/a0019004

Osiurak, F., Lasserre, S., Arbanti, J., Brogniart, J., Bluet, A., Navarro, J., Reynaud, E., 2021b. Technical reasoning is necessary not to reinvent the wheel. Nat. Hum. Behav. In press.

Osiurak, F., Lesourd, M., Navarro, J., Reynaud, E., 2020b. Technition: When tools come out of the closet. Perspect. Psychol. Sci. 15, 880-897. https://doi.org/10.1177/1745691620902145

Osiurak, F., Reynaud, E., 2020a. The elephant in the room: What matters cognitively in cumulative technological culture. Behav. Brain Sci. 43, e156. https://doi.org/10.1017/S0140525X19003236

Osiurak, F., Reynaud, E., 2020b. The elephant in the China shop: When technical reasoning meets cumulative technological culture. Behav. Brain Sci. 43. https://doi.org/10.1017/S0140525X20000291

Osiurak, F., Rossetti, Y., Badets, A., 2017. What is an affordance? 40 years later. Neurosci. Biobehav. Rev. 77, 403-417. https://doi.org/10.1016/j.neubiorev.2017.04.014

Pagano, C.C., Day, B., 2020. Ecological interface design inspired by 'The meaningful environment,' in: Wagman, J.B., Blau, J.J.C. (Eds.), Perception as Information Detection: Reflections on Gibson's Ecological Approach to Visual Perception. Routledge, New York, NY, pp. 37-50.

Pagano, C.C., Fitzpatrick, P., Turvey, M.T., 1993. Tensorial basis to the constancy of perceived object extent over variations of dynamic touch. Percept. Psychophys. 54, 43-54. https://doi.org/10.3758/BF03206936

Pagano, C.C., Turvey, M.T., 1998. Eigenvectors of the inertia tensor and perceiving the orientations of limbs and objects. J. Appl. Biomech. 14, 331-359. https://doi.org/10.1123/jab.14.4.331 10.1123/jab.14.4.331

Pagnotta, M., Laland, K.N., Coco, M.I., 2020. Attentional coordination in demonstrator-observer dyads facilitates learning and predicts performance in a novel manual task. Cognition 201, 104314. https://doi.org/10.1016/j.cognition.2020.104314 
1989

1990

1991

1992

1993

1994

1995

1996

1997

1998

1999

2000

2001

2002

2003

2004

2005

2006

2007

2008

2009

2010

2011

2012

2013

2014

2015

2016

2017

2018

2019

2020

2021

2022

2023

2024

2025

2026

Pan, Y., Dikker, S., Goldstein, P., Zhu, Y., Yang, C., Hu, Y., 2020. Instructor-learner brain coupling discriminates between instructional approaches and predicts learning. Neuroimage 211, 116657. https://doi.org/10.1016/j.neuroimage.2020.116657

Pargeter, J., Khreisheh, N., Stout, D., 2019. Understanding stone tool-making skill acquisition: Experimental methods and evolutionary implications. J. Hum. Evol. 133, 146-166. https://doi.org/10.1016/j.jhevol.2019.05.010

Parker, S.T., Gibson, K.R., 1977. Object manipulation, tool use and sensorimotor intelligence as feeding adaptations in cebus monkeys and great apes. J. Hum. Evol. 6, 623-641. https://doi.org/10.1016/S0047-2484(77)80135-8

Pazzaglia, M., Molinari, M., 2016. The embodiment of assistive devices-from wheelchair to exoskeleton. Phys. Life Rev. 16, 163-175. https://doi.org/10.1016/j.plrev.2015.11.006

Powers, S.T., van Schaik, C.P., Lehmann, L., 2016. How institutions shaped the last major evolutionary transition to large-scale human societies. Philos. Trans. R. Soc. B Biol. Sci. 371, 20150098. https://doi.org/10.1098/rstb.2015.0098

Prat, C.S., Madhyastha, T.M., Mottarella, M.J., Kuo, C.-H., 2020. Relating natural language aptitude to individual differences in learning programming languages. Sci. Rep. 10, 3817. https://doi.org/10.1038/s41598-020-60661-8

Preston, B., 2012. A Philosophy of Material Culture: Action, Function, and Mind. Routledge, London, UK.

Profeta, V.L.S., Turvey, M.T., 2018. Bernstein's levels of movement construction: A contemporary perspective. Hum. Mov. Sci. 57, 111-133. https://doi.org/10.1016/j.humov.2017.11.013

Profeta, V.L.S., Turvey, M.T., Carello, C., 2020. Goal-directed action and the architecture of movement organization, in: Latash, M.L. (Ed.), Bernstein's Construction of Movements. Routledge, New York, NY, pp. 308-319.

Pruetz, J.D., Bertolani, P., 2007. Savanna chimpanzees, Pan troglodytes verus, hunt with tools. Curr. Biol. 17, 412-417. https://doi.org/10.1016/j.cub.2006.12.042

Pryor, C., Perfors, A., Howe, P.D.L., 2019. Even arbitrary norms influence moral decision-making. Nat. Hum. Behav. 3, 57-62. https://doi.org/10.1038/s41562-018-0489-y

Ptak, R., Schnider, A., Fellrath, J., 2017. The dorsal frontoparietal network: A core system for emulated action. Trends Cogn. Sci. 21, 589-599. https://doi.org/10.1016/j.tics.2017.05.002

Rączaszek-Leonardi, J., Nomikou, I., Rohlfing, K.J., Deacon, T.W., 2018. Language development from an ecological perspective: Ecologically valid ways to abstract symbols. Ecol. Psychol. 30, 39-73. https://doi.org/10.1080/10407413.2017.1410387

Rein, R., Bril, B., Nonaka, T., 2013. Coordination strategies used in stone knapping. Am. J. Phys. Anthropol. 150, 539-550. https://doi.org/10.1002/ajpa.22224

Reindl, E., Apperly, I.A., Beck, S.R., Tennie, C., 2017. Young children copy cumulative technological design in the absence of action information. Sci. Rep. 7, 1788. https://doi.org/10.1038/s41598017-01715-2 
Renfrew, C., Frith, C., Malafouris, L., Frey, S.H., 2008. Tool use, communicative gesture and cerebral asymmetries in the modern human brain. Philos. Trans. R. Soc. B Biol. Sci. 363, 1951-1957. https://doi.org/10.1098/rstb.2008.0008

Reynaud, E., Lesourd, M., Navarro, J., Osiurak, F., 2016. On the neurocognitive origins of human tool use: A critical review of neuroimaging data. Neurosci. Biobehav. Rev. 64, 421-437. https://doi.org/10.1016/j.neubiorev.2016.03.009

Reynaud, E., Navarro, J., Lesourd, M., Osiurak, F., 2019. To watch is to work: A review of neuroimaging data on tool use observation network. Neuropsychol. Rev. 29, 484-497. https://doi.org/10.1007/s11065-019-09418-3

Richardson, M.J., Shockley, K., Fajen, B.R., Riley, M.A., Turvey, M.T., 2008. Ecological psychology: Six principles for an embodied-embedded approach to behavior, in: Calvo, P., Gomila, A.B.T.-H. of C.S. (Eds.), Perspectives on Cognitive Science. Elsevier, San Diego, pp. 159-187. https://doi.org/10.1016/B978-0-08-046616-3.00009-8

Riley, M., Richardson, M., Shockley, K., Ramenzoni, V., 2011. Interpersonal synergies. Front. Psychol. 2, 38. https://doi.org/10.3389/fpsyg.2011.00038

Rothi, L.J.G., Ochipa, C., Heilman, K.M., 1991. A Cognitive neuropsychological model of limb praxis. Cogn. Neuropsychol. 8, 443-458. https://doi.org/10.1080/02643299108253382

Rutz, C., Hunt, G.R., 2020. New Caledonian crows afford invaluable comparative insights into human cumulative technological culture. Behav. Brain Sci. 43, e177. https://doi.org/10.1017/S0140525X20000187

Salazar-López, E., Schwaiger, B.J., Hermsdörfer, J., 2016. Lesion correlates of impairments in actual tool use following unilateral brain damage. Neuropsychologia 84, 167-180. https://doi.org/10.1016/j.neuropsychologia.2016.02.007

Sánchez, C.C., Moreno, F.J., Vaíllo, R.R., Romero, A.R., Coves, Á., Murillo, D.B., 2017. The role of motor variability in motor control and learning depends on the nature of the task and the individual's capabilities. Eur. J. Hum. Mov. 38, 12-26.

Sanz, C.M., Morgan, D.B., 2007. Chimpanzee tool technology in the Goualougo Triangle, Republic of Congo. J. Hum. Evol. 52, 420-433. https://doi.org/10.1016/j.jhevol.2006.11.001

Schaap, P., 2021. From environmental sensing to developmental control: Cognitive evolution in dictyostelid social amoebas. Philos. Trans. R. Soc. B Biol. Sci. 376, 20190756. https://doi.org/10.1098/rstb.2019.0756

Schirmer, A., Fairhurst, M., Hoehl, S., 2021. Being 'in sync'-is interactional synchrony the key to understanding the social brain? Soc. Cogn. Affect. Neurosci. 16, 1-4. https://doi.org/10.1093/scan/nsaa148

Scholz, J.P., Schöner, G., 1999. The uncontrolled manifold concept: identifying control variables for a functional task. Exp. Brain Res. 126, 289-306. https://doi.org/10.1007/s002210050738

Scholz, J.P., Schöner, G., Latash, M.L., 2000. Identifying the control structure of multijoint coordination during pistol shooting. Exp. Brain Res. 135, 382-404. https://doi.org/10.1007/s002210000540 
2065

2066

2067

2068

2069

2070

2071

2072

2073

2074

2075

2076

2077

2078

2079

2080

2081

2082

2083

2084

2085

2086

2087

2088

2089

2090

2091

2092

2093

2094

2095

2096

2097

2098

2099

2100

Schöner, G., 1995. Recent developments and problems in human movement science and their conceptual implications. Ecol. Psychol. 7, 291-314. https://doi.org/10.1207/s15326969eco0704_5

Seed, A., Emery, N., Clayton, N., 2009. Intelligence in corvids and apes: A case of convergent evolution? Ethology 115, 401-420. https://doi.org/10.1111/j.1439-0310.2009.01644.x

Shamay-Tsoory, S.G., Saporta, N., Marton-Alper, I.Z., Gvirts, H.Z., 2019. Herding brains: A core neural mechanism for social alignment. Trends Cogn. Sci. 23, 174-186. https://doi.org/10.1016/j.tics.2019.01.002

Shaw, R., 2001. Processes, acts, and experiences: Three stances on the problem of intentionality. Ecol. Psychol. 13, 275-314. https://doi.org/10.1207/S15326969ECO1304_02

Shaw, R.E., Flascher, O.M., Kadar, E.E., 1995. Dimensionless invariants for intentional systems: Measuring the fit of vehicular activities to environmental layout, in: Flach, J.M., Hancock, P.A., Caird, J., Vicente, K.J. (Eds.), Global Perspectives on the Ecology of Human-Machine Systems. Lawrence Erlbaum, Hillsdale, NJ, pp. 293-357.

Shea, N., Boldt, A., Bang, D., Yeung, N., Heyes, C., Frith, C.D., 2014. Supra-personal cognitive control and metacognition. Trends Cogn. Sci. 18, 186-193. https://doi.org/10.1016/j.tics.2014.01.006

Shumaker, R.W., Walkup, K.R., Beck, B., 2011. Animal Tool Behavior: The Use and Manufacture of Tools by Animals. Johns Hopkins University Press, Baltimore, MD.

Silveri, M.C., Ciccarelli, N., 2009. Semantic memory in object use. Neuropsychologia 47, 2634-2641. https://doi.org/10.1016/j.neuropsychologia.2009.05.013

Sirigu, A., Duhamel, J.-R., Poncet, M., 1991. The role of sensorimotor experience in object recognition: A case of multimodal agnosia. Brain 114, 2555-2573. https://doi.org/10.1093/brain/114.6.2555

Smitsman, A.W., 1997. The development of tool use: Changing boundaries between organism and environment, in: Dent-Read, C., Zukow-Goldring, P. (Eds.), Evolving Explanations of Development. American Psychological Association, Washington, DC, pp. 301-329.

Smitsman, A.W., Cox, R.F.A., Bongers, R.M., 2005. Action dynamics in tool use, in: Roux, V., Bril, B. (Eds.), Stone Knapping: The Necessary Conditions for a Uniquely Hominid Behaviour. McDonald Institute for Archaeological Research, Cambridge, UK, pp. 129-144.

Sokolov, A.A., Miall, R.C., Ivry, R.B., 2017. The cerebellum: Adaptive prediction for movement and cognition. Trends Cogn. Sci. 21, 313-332. https://doi.org/10.1016/j.tics.2017.02.005

Stephen, D.G., Arzamarski, R., Michaels, C.F., 2010. The role of fractality in perceptual learning: Exploration in dynamic touch. J. Exp. Psychol. Hum. Percept. Perform. 36, 1161-1173. https://doi.org/10.1037/a0019219

Stephen, D.G., Boncoddo, R.A., Magnuson, J.S., Dixon, J.A., 2009. The dynamics of insight: Mathematical discovery as a phase transition. Mem. Cognit. 37, 1132-1149. https://doi.org/10.3758/MC.37.8.1132 
2101 Stephen, D.G., Dixon, J.A., 2011. Strong anticipation: Multifractal cascade dynamics modulate scaling

in synchronization behaviors. Chaos, Solitons \& Fractals 44, 160-168. https://doi.org/10.1016/j.chaos.2011.01.005

Stephen, D.G., Hajnal, A., 2011. Transfer of calibration between hand and foot: Functional equivalence and fractal fluctuations. Attention, Perception, Psychophys. 73, 1302-1328. https://doi.org/10.3758/s13414-011-0142-6

Sterelny, K., 2012. The Evolved Apprentice: How Evolution Made Humans Unique. MIT Press, Cambridge, MA.

Stoffregen, T.A., Mantel, B., Bardy, B.G., 2017. The senses considered as one perceptual system. Ecol. Psychol. 29, 165-197. https://doi.org/10.1080/10407413.2017.1331116

Stoffregen, T.A., Pittenger, J.B., 1995. Human echolocation as a basic form of perception and action. Ecol. Psychol. 7, 181-216. https://doi.org/10.1207/s15326969eco0703_2

Stoinski, T.S., Beck, B.B., 2001. Spontaneous tool use in captive, free-ranging golden lion tamarins (Leontopithecus rosalia rosalia). Primates 42, 319-326. https://doi.org/10.1007/BF02629623

Stout, D., 2013. Neuroscience of technology, in: Richerson, P.J., Christiansen, M. (Eds.), Cultural Evolution: Society, Technology, Language, and Religion. MIT Press, Cambridge, MA, pp. 157173.

Stout, D., Chaminade, T., Thomik, A., Apel, J., Faisal, A., 2018. Grammars of action in human behavior and evolution. bioRxiv 281543. https://doi.org/10.1101/281543

Stout, D., Hecht, E., Khreisheh, N., Bradley, B., Chaminade, T., 2015. Cognitive demands of lower paleolithic toolmaking. PLoS One 10, e0121804.

Stout, D., Hecht, E.E., 2017. Evolutionary neuroscience of cumulative culture. Proc. Natl. Acad. Sci. 114, 7861-7868. https://doi.org/10.1073/pnas.1620738114

Suddendorf, T., Brinums, M., Imuta, K., 2016. Shaping one's future self-The development of deliberate practice, in: Michaelian, K., Klein, S.B., Szpunar, K.K. (Eds.), Seeing the Future: Theoretical Perspectives on Future-Oriented Mental Time Travel. Oxford Univeristy Press, pp. 343-366.

Suddendorf, T., Bulley, A., Miloyan, B., 2018. Prospection and natural selection. Curr. Opin. Behav. Sci. 24, 26-31. https://doi.org/10.1016/j.cobeha.2018.01.019

Takahashi, C., Watt, S.J., 2017. Optimal visual-haptic integration with articulated tools. Exp. Brain Res. 235, 1361-1373. https://doi.org/10.1007/s00221-017-4896-5

Taylor, A.H., Hunt, G.R., Holzhaider, J.C., Gray, R.D., 2007. Spontaneous metatool use by New Caledonian crows. Curr. Biol. 17, 1504-1507. https://doi.org/10.1016/j.cub.2007.07.057

Taylor, A.H., Jelbert, S., 2020. The crow in the room: New Caledonian crows offer insight into the necessary and sufficient conditions for cumulative cultural evolution. Behav. Brain Sci. 43, e178. https://doi.org/10.1017/S0140525X20000102

Teng, D.W., Eddy, C.L., Kelty-Stephen, D.G., 2016. Non-visually-guided distance perception depends on matching torso fluctuations between training and test. Attention, Perception, Psychophys. 78, 2320-2328. https://doi.org/10.3758/s13414-016-1213-5 
Tennie, C., Call, J., Tomasello, M., 2009. Ratcheting up the ratchet: on the evolution of cumulative culture. Philos. Trans. R. Soc. B Biol. Sci. 364, 2405-2415. https://doi.org/10.1098/rstb.2009.0052

Thomas, B.J., Riley, M.A., Wagman, J.B., 2019. Information and its detection: The consequences of Gibson's theory of information pickup, in: Wagman, J.B., Blau, J.J.C. (Eds.), Perception as Information Detection: Reflections on Gibson's Ecological Approach to Visual Perception. Routledge, New York, NY, pp. 237-252.

Tomasello, M., Kruger, A.C., Ratner, H.H., 1993. Cultural learning. Behav. Brain Sci. 16, 495-511. https://doi.org/10.1017/S0140525X0003123X

Tonooka, R., 2001. Leaf-folding behavior for drinking water by wild chimpanzees (Pan troglodytes verus) at Bossou, Guinea. Anim. Cogn. 4, 325-334. https://doi.org/10.1007/s100710100110

Troscianko, J., von Bayern, A.M.P., Chappell, J., Rutz, C., Martin, G.R., 2012. Extreme binocular vision and a straight bill facilitate tool use in New Caledonian crows. Nat. Commun. 3, 1110. https://doi.org/10.1038/ncomms2111

Truskanov, N., Prat, Y., 2018. Cultural transmission in an ever-changing world: Trial-and-error copying may be more robust than precise imitation. Philos. Trans. R. Soc. B Biol. Sci. 373, 20170050. https://doi.org/10.1098/rstb.2017.0050

Tuitert, I., Golenia, L., Otten, E., Bootsma, R.J., Bongers, R.M., 2020. Task constraints act at the level of synergies and at the level of end-effector kinematics in manual reaching and manual lateral interception. J. Exp. Psychol. Hum. Percept. Perform. 46, 1511-1526. https://doi.org/10.1037/xhp0000861

Tulving, E., 1985. Memory and consciousness. Can. Psychol. 26, 1-12. https://doi.org/10.1037/h0080017

Turvey, M.T., 2018. Lectures on Perception: An Ecological Perspective. Routledge, New York, NY.

Turvey, M.T., 2007. Action and perception at the level of synergies. Hum. Mov. Sci. 26, 657-697. https://doi.org/10.1016/j.humov.2007.04.002

Turvey, M.T., 1992. Affordances and prospective control: An outline of the ontology. Ecol. Psychol. 4, 173-187. https://doi.org/10.1207/s15326969eco0403_3

Turvey, M.T., 1990. Coordination. Am. Psychol. 45, 938-953. https://doi.org/10.1037/0003066X.45.8.938

Turvey, M.T., Fonseca, S.T., 2014. The medium of haptic perception: A tensegrity hypothesis. J. Mot. Behav. 46, 143-187. https://doi.org/10.1080/00222895.2013.798252

Tylén, K., Philipsen, J.S., Roepstorff, A., Fusaroli, R., 2016. Trails of meaning construction: Symbolic artifacts engage the social brain. Neuroimage 134, 105-112. https://doi.org/10.1016/j.neuroimage.2016.03.056

Umiltà, M.A., Escola, L., Intskirveli, I., Grammont, F., Rochat, M., Caruana, F., Jezzini, A., Gallese, V., Rizzolatti, G., 2008. When pliers become fingers in the monkey motor system. Proc. Natl. Acad. Sci. USA 105, 2209-2213. https://doi.org/10.1073/pnas.0705985105 
Vaesen, K., 2012. The cognitive bases of human tool use. Behav. Brain Sci. 35, 203-218. https://doi.org/10.1017/S0140525X11001452

Valk, T.A., Mouton, L.J., Bongers, R.M., 2016. Joint-angle coordination patterns ensure stabilization of a body-plus-tool system in point-to-point movements with a rod. Front. Psychol. 7, 826. https://doi.org/10.3389/fpsyg.2016.00826

van den Herik, J.C., 2021. Rules as resources: An ecological-enactive perspective on linguistic normativity. Phenomenol. Cogn. Sci. 20, 93-116. https://doi.org/10.1007/s11097-020-09676-0

van der Steen, M.C., Bongers, R.M., 2011. Joint angle variability and co-variation in a reaching with a rod task. Exp. Brain Res. 208, 411-422. https://doi.org/10.1007/s00221-010-2493-y

van Dijk, L., Bongers, R.M., 2014. The emergence of an action system: The organization of gaze in creating novel tools. Ecol. Psychol. 26, 177-197. https://doi.org/10.1080/10407413.2014.929476

van Elk, M., van Schie, H., Bekkering, H., 2014. Action semantics: A unifying conceptual framework for the selective use of multimodal and modality-specific object knowledge. Phys. Life Rev. 11, 220250. https://doi.org/10.1016/j.plrev.2013.11.005

van Schaik, C.P., Burkart, J.M., 2011. Social learning and evolution: The cultural intelligence hypothesis. Philos. Trans. R. Soc. B Biol. Sci. 366, 1008-1016. https://doi.org/10.1098/rstb.2010.0304

Vendetti, M.S., Bunge, S.A., 2014. Evolutionary and developmental changes in the lateral frontoparietal network: A little goes a long way for higher-level cognition. Neuron 84, 906-917. https://doi.org/10.1016/j.neuron.2014.09.035

Vingerhoets, G., 2014. Contribution of the posterior parietal cortex in reaching, grasping, and using objects and tools. Front. Psychol. 5, 151. https://doi.org/10.3389/fpsyg.2014.00151

Wagman, J.B., 2020. A guided tour of Gibson's theory of affordances, in: Wagman, J.B., Blau, J.J.C. (Eds.), Perception as Information Detection: Reflections on Gibson's Ecological Approach to Visual Perception. Routledge, New York, NY, pp. 130-148.

Wagman, J.B., Caputo, S.E., Stoffregen, T.A., 2016. Hierarchical nesting of affordances in a tool use task. J. Exp. Psychol. Hum. Percept. Perform. 42, 1627-1642. https://doi.org/10.1037/xhp0000251

Wagman, J.B., Carello, C., 2003. Haptically creating affordances: The user-tool interface. J. Exp. Psychol. Appl. 9, 175-186. https://doi.org/10.1037/1076-898X.9.3.175

Wagman, J.B., Carello, C., 2001. Affordances and inertial constraints on tool use. Ecol. Psychol. 13, 173-195. https://doi.org/10.1207/S15326969ECO1303_1

Wagman, J.B., Hajnal, A., 2016. Use your head! Perception of action possibilities by means of an object attached to the head. Exp. Brain Res. 234, 829-836. https://doi.org/10.1007/s00221-0154509-0

Wagman, J.B., Hajnal, A., 2014a. Task specificity and anatomical independence in perception of properties by means of a wielded object. J. Exp. Psychol. Hum. Percept. Perform. 40, 23722391. https://doi.org/10.1037/xhp0000014 
Wagman, J.B., Hajnal, A., 2014b. Getting off on the right (or left) foot: Perceiving by means of a rod attached to the preferred or non-preferred foot. Exp. Brain Res. 232, 3591-3599. https://doi.org/10.1007/s00221-014-4047-1

Wagman, J.B., Langley, M.D., Farmer-Dougan, V., 2018. Carrying their own weight: Dogs perceive changing affordances for reaching. Q. J. Exp. Psychol. 71, 1040-1044. https://doi.org/10.1080/17470218.2017.1322990

Wagman, J.B., Langley, M.D., Higuchi, T., 2017. Turning perception on its head: Cephalic perception of whole and partial length of a wielded object. Exp. Brain Res. 235, 153-167. https://doi.org/10.1007/s00221-016-4778-2

Wagman, J.B., Lozano, S., Jiménez, A., Covarrubias, P., Cabrera, F., 2019. Perception of affordances in the animal kingdom and beyond, in: Zepeda, I., Camacho, J., Camacho, E. (Eds.), Aproximaciones Al Estudio Del Comportamiento y Sus Aplicaciones. Universidad de Guadalajara, Ocotlátan, Mexico, pp. 70-108.

Wagman, J.B., Miller, D.B., 2003. Nested reciprocities: The organism-environment system in perception-action and development. Dev. Psychobiol. 42, 317-334. https://doi.org/10.1002/dev.10114

Wagman, J.B., Taylor, K.R., 2005. Perceiving affordances for aperture crossing for the person-plusobject system. Ecol. Psychol. 17, 105-130. https://doi.org/10.1207/s15326969eco1702_3

Walsh, P.T., Hansell, M., Borello, W.D., Healy, S.D., 2011. Individuality in nest building: Do Southern Masked weaver (Ploceus velatus) males vary in their nest-building behaviour? Behav. Processes 88, 1-6. https://doi.org/10.1016/j.beproc.2011.06.011

Warren, W.H., 2005. Direct perception: The view from here. Philos. Top. 33, 335-361.

Warren, W.H., 1984. Perceiving affordances: Visual guidance of stair climbing. J. Exp. Psychol. Hum. Percept. Perform. 10, 683-703. https://doi.org/10.1037/0096-1523.10.5.683

Watanabe, K., Urasopon, N., Malaivijitnond, S., 2007. Long-tailed macaques use human hair as dental floss. Am. J. Primatol. 69, 940-944. https://doi.org/10.1002/ajp.20403

Wheaton, L.A., Hallett, M., 2007. Ideomotor apraxia: A review. J. Neurol. Sci. 260, 1-10. https://doi.org/10.1016/j.jns.2007.04.014

Whiten, A., 2015. Experimental studies illuminate the cultural transmission of percussive technologies in Homo and Pan. Philos. Trans. R. Soc. B Biol. Sci. 370, 20140359. https://doi.org/10.1098/rstb.2014.0359

Wilson, J.A., 2000. Ontological butchery: Organism concepts and biological generalizations. Philos. Sci. 67, S301-S311. https://doi.org/10.1086/392827

Wissing, M.B.G., Golenia, L., Smith, J., Bongers, R.M., 2020. Adjustments in end-effector trajectory and underlying joint angle synergies after a target switch: Order of adjustment is flexible. PLoS One 15, e0238561. https://doi.org/10.1371/journal.pone.0238561 
2251 Wood, E.H., Tang, P.H., De la Huerta, I., Korot, E., Muscat, S., Palanker, D.A., Williams, G.A., 2019. 2252 Stem cell therapies, gene-based therapies, optogenetics, and retinal prosthetics: Current state 2253 and implications for the future. RETINA 39. https://doi.org/10.1097/IAE.0000000000002449

2254 Yildirim, I., Wu, J., Kanwisher, N., Tenenbaum, J., 2019. An integrative computational architecture for 2255 object-driven cortex. Curr. Opin. Neurobiol. 55, 73-81. https://doi.org/10.1016/j.conb.2019.01.010

2256 Zach, R., 1978. Selection and dropping of whelks by Northwestern crows. Behaviour 67, 134-147. $2257 \quad$ https://doi.org/10.1163/156853978X00297

2258 Ziegenhorn, M.A., 2016. Best dressed test: A study of the covering behavior of the collector urchin, 2259 Tripneustes gratilla. PLoS One 11, e0153581. https://doi.org/10.1371/journal.pone.0153581

2260 Zorick, T., Landers, J., Leuchter, A., Mandelkern, M.A., 2020. EEG multifractal analysis correlates with 2261 cognitive testing scores and clinical staging in mild cognitive impairment. J. Clin. Neurosci. 76, 2262 195-200. https://doi.org/10.1016/j.jocn.2020.04.003

2263 Zwirner, E., Thornton, A., 2015. Cognitive requirements of cumulative culture: Teaching is useful but 2264 not essential. Sci. Rep. 5, 16781. https://doi.org/10.1038/srep16781 
2265 Table 1. Definitions of tool use, tool making and construction according to Shumaker et al. (2011).

Label Definition

Tool use

The external employment of an unattached or manipulable attached environmental object to alter more efficiently the form, position, or condition of another object, another organism, or the user itself, when the user holds and directly manipulates the tool during or prior to use and is responsible for the proper and effective orientation of the tool (p. 5).

Tool making Structural modification of an object or an existing tool by the user or a conspecific so that the object/tool serves, or serves more effectively, as a tool (p. 11).

Construction Two or more tools and/or objects physically linked to make a functional, semipermanent thing that, once completed, is not held or directly manipulated in its entirety. A construction itself is therefore not a tool. Nor is it tool manufacture, because the product is not a tool (p. 19). 
Table 2. Example behaviors identified in the literature as tool use, categorized as 'Instrumental problem solving' or 'Tooling' according to Fragaszy and Mangalam's (2018) definition of tool use. Adapted from Fragaszy and Mangalam (2018).

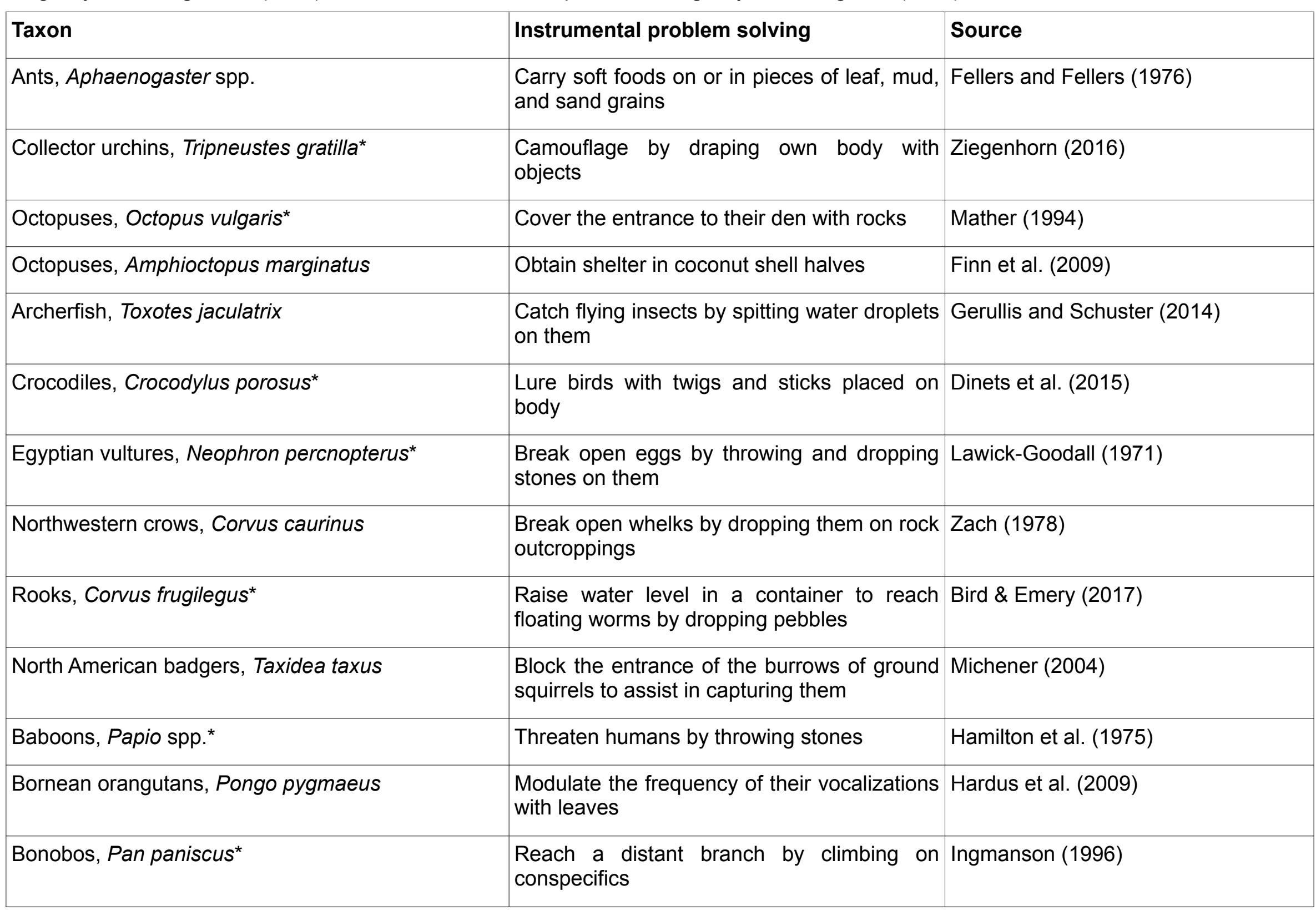




\begin{tabular}{|c|c|c|}
\hline Chimpanzees, Pan troglodytes & $\begin{array}{l}\text { Reach for a hanging banana by stepping on } \\
\text { wooden boxes }\end{array}$ & Köhler (1925) \\
\hline Chimpanzees, Pan troglodytes ${ }^{*}$ & $\begin{array}{l}\text { Drink water from tree hollows using leaves to } \\
\text { sponge up the water }\end{array}$ & Tonooka (2001) \\
\hline Taxon & Tooling & Source \\
\hline New Caledonian crows, Corvus moneduloides* & $\begin{array}{l}\text { Extract insect larvae from cavities in } \\
\text { branches with probes }\end{array}$ & Hunt (Hunt, 1996) \\
\hline Hyacinth macaws, Anodorhynchus hyacinthinus & Stabilize a nut in the beak with wood wedges & Borsari and Ottoni (2005) \\
\hline Lion, Panthera leo & $\begin{array}{l}\text { Rub a sore area of its paw using a thorn held } \\
\text { in the mouth }\end{array}$ & Bauer (2001) \\
\hline Elephants, Elephus maximus & $\begin{array}{l}\text { Brush flies off their body with branches held } \\
\text { in the trunk }\end{array}$ & Hart et al. (2001) \\
\hline Bearded capuchin monkeys, Sapajus libidinosus * & Dig soil with stones to excavate tubers & Falótico and Ottoni (2016) \\
\hline Long-tailed macaques, Macaca fascicularis & Floss their teeth with hair & Watanabe et al. (2007) \\
\hline Sumatran orangutans, Pongo abelii ${ }^{\star}$ & Wipe body with leaves to remove substance & Fox et al. (1999) \\
\hline Chimpanzees, Pan troglodytes* & $\begin{array}{l}\text { Flush or disable vertebrate prey in tree } \\
\text { hollow with stick }\end{array}$ & Pruetz and Bertolani (2007) \\
\hline Chimpanzees, Pan troglodytes & $\begin{array}{l}\text { Puncture soil with stick to reach underground } \\
\text { termite nests }\end{array}$ & Sanz and Morgan (2007) \\
\hline Golden lion tamarin, Leontopithecus rosalia* & Groom conspecific with stick & Stoinski and Beck (2001) \\
\hline
\end{tabular}

${ }^{*}$ Additional taxa have been reported to behave in similar ways. 

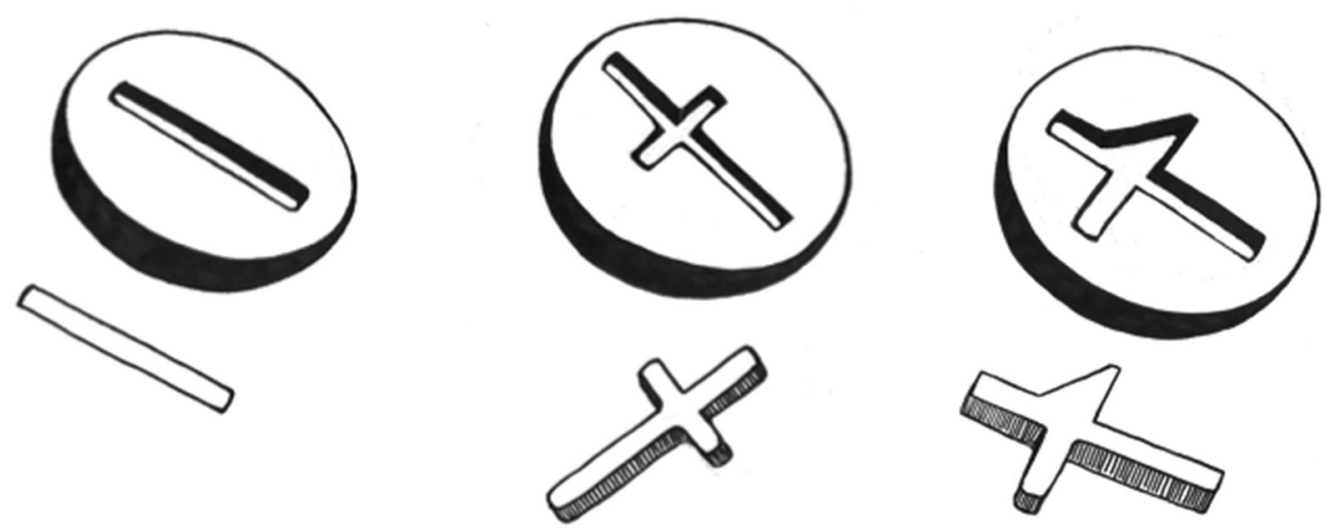

2271

2272

2273

2274

2275

2276

2277

2278

Fig. 1. Drawing of bar, cross, tomahawk shapes, and the respective cutouts presented to two-, three-, and four-year-old children, adult tufted capuchin monkeys (Sapajus spp.), and adult chimpanzees (Pan troglodytes). Two- and three-year-old children routinely aligned a bar-shaped stick and a crossshaped stick to its matching cutout, and four-year-old children can also align a tomahawk-shaped stick, which entails attending to multiple spatial features concurrently, but capuchin monkeys and chimpanzees faced severe difficulties in aligning even a simple bar-shaped stick to its matching cutout. See Fragaszy et al. (2011), la Bour et al. (2014), and Fragaszy et al. (2015) for details. Drawing courtesy of $D$. Sharpe. 

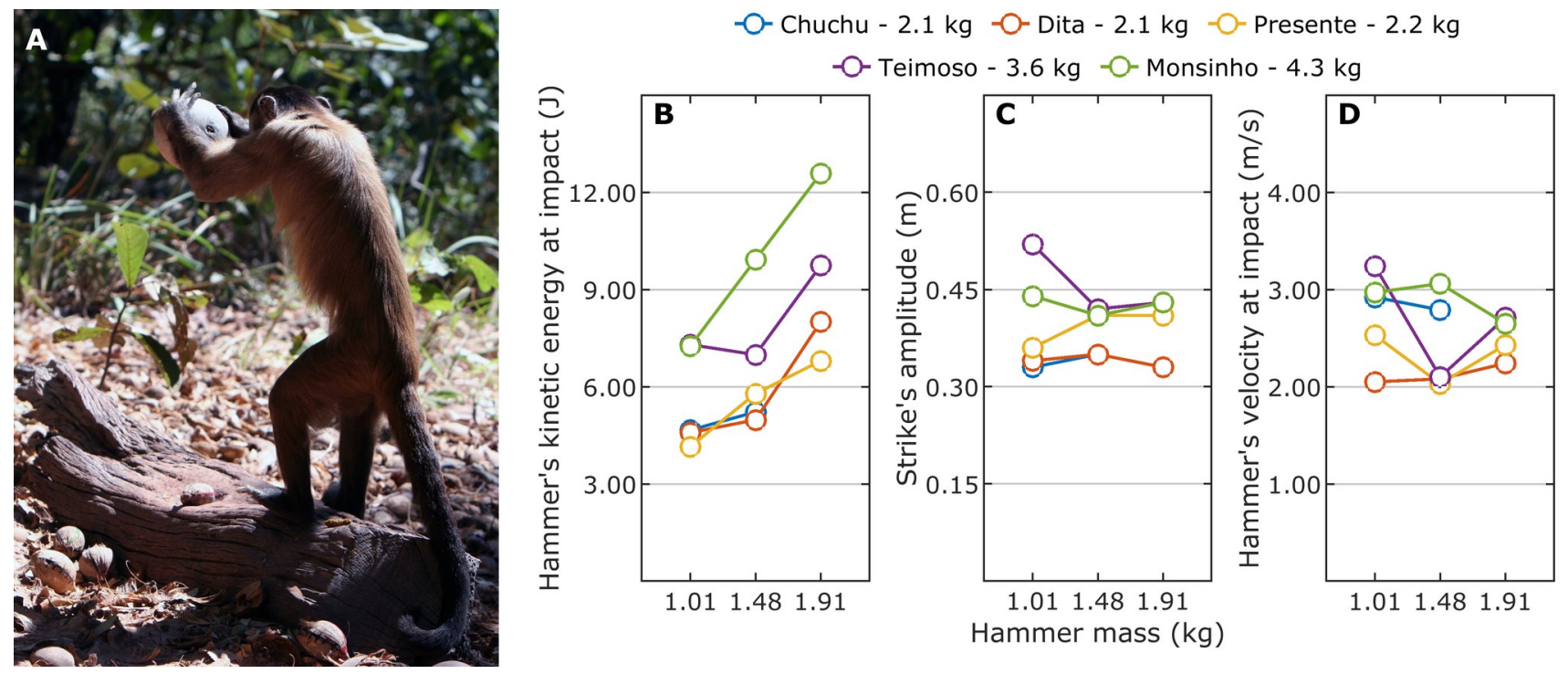

2280 Fig. 2. When cracking nuts using stone hammers, wild bearded capuchin monkeys (Sapajus 2281 libidinosus) alter their strikes to strike a nut with less or more force based on the type and condition of 2282 the nut; they do so by adjusting the amplitude [and velocity] of the strike but do not adjust the 2283 hammer's kinetic energy at impact. (a) An adult monkey is striking an intact piaçava nut (inset) 2284 placed in a pit on a log anvil-with a quartzite stone hammer. Photo courtesy of Barth A. Wright. (b) 2285 Hammer's kinetic energy at impact. (c) Strike's amplitude. (d) Hammer's velocity at impact. Adapted 2286 from Mangalam, Pacheco, et al. (2018). 


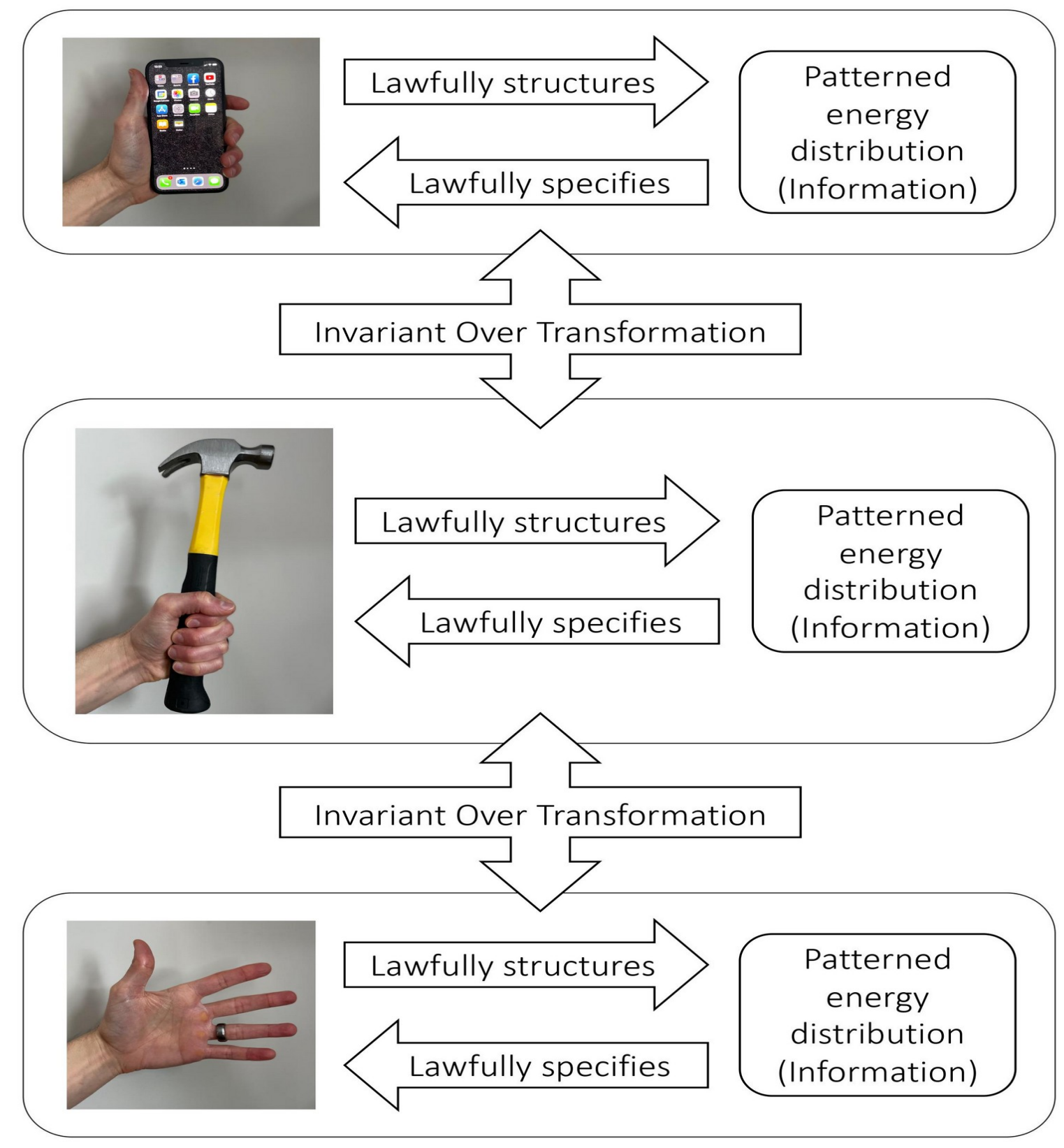

2288 Fig. 3. The relationship between animal and environment lawfully structures patterned energy array so 2289 as to provide information about this relationship (bottom). Such lawfulness entails that information 2290 about a given affordance is invariant over transformations including whether or not a tool alters the fit 2291 between animal and environment (middle) and whether or not the tool is virtual (top). 


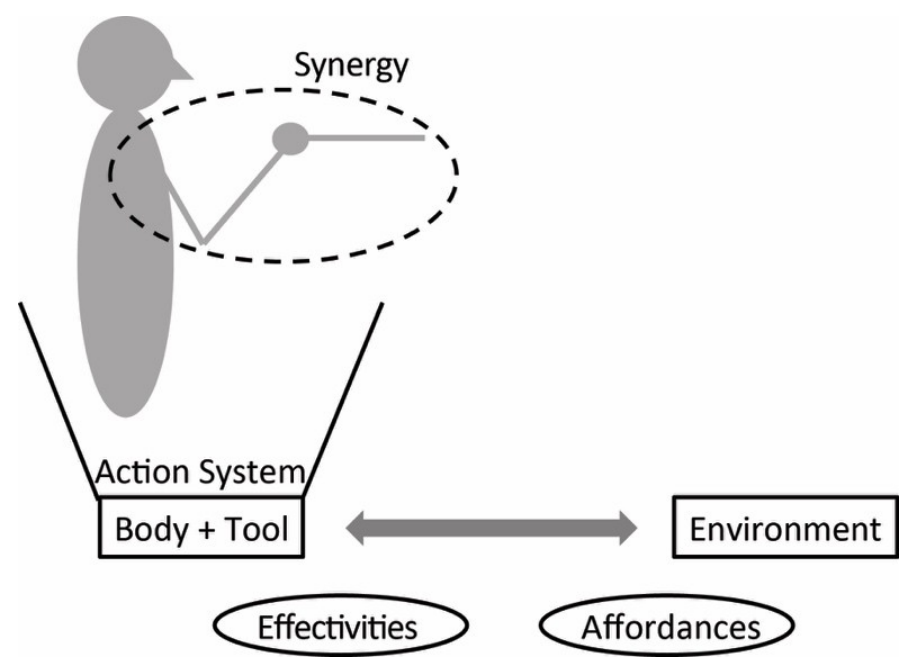

2293 Fig. 4. The tool in the hand of the use is schematically depicted. The tool affects the synergies in the 2294 action system, and therefore the effectivities and the affordances. 


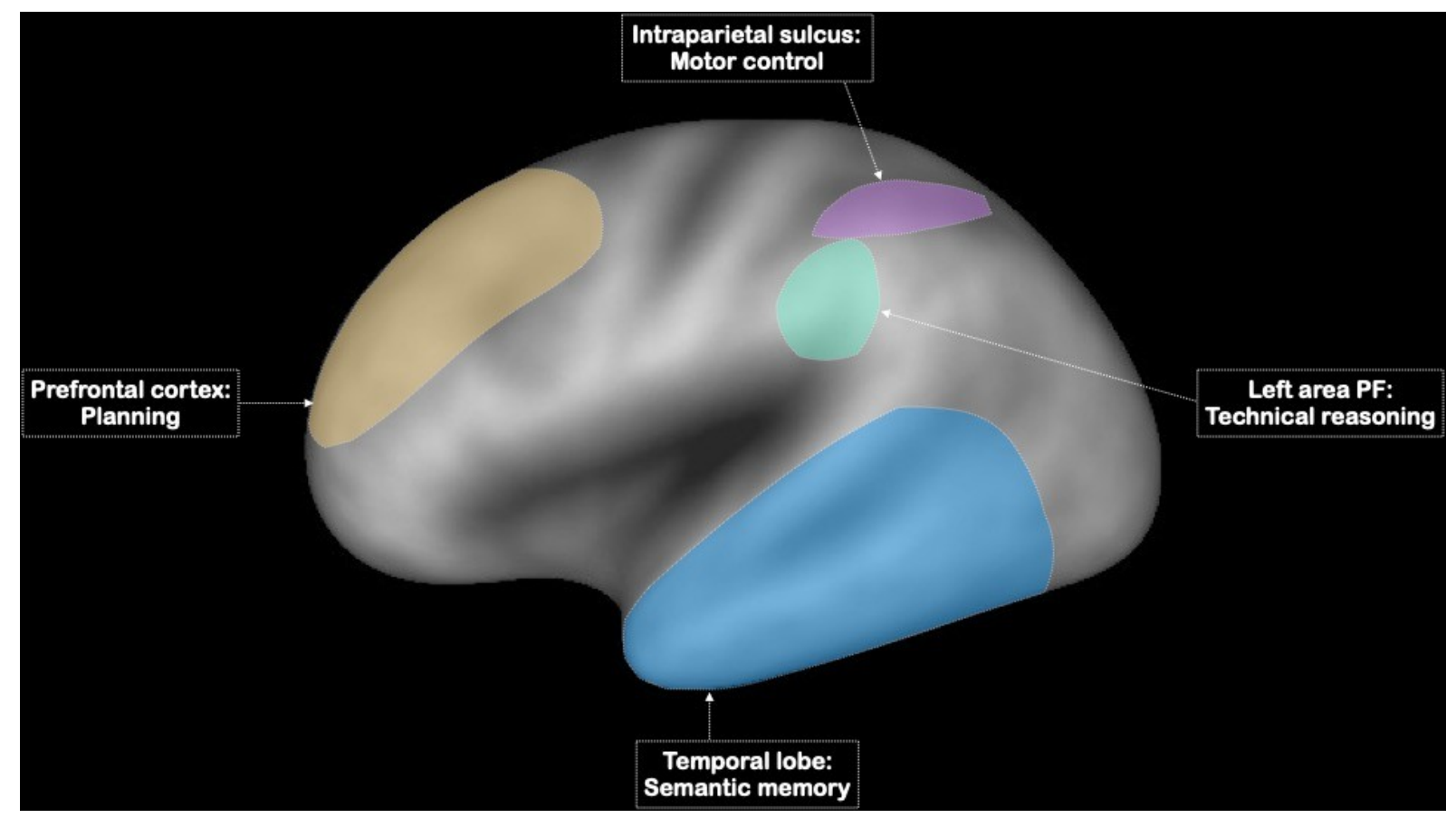

2296 Fig. 5. Neurocognitive functions involved in tool use according to the technical-reasoning hypothesis. 